\title{
Covariant cosmological quantum space-time, higher-spin and gravity in the IKKT matrix model
}

\author{
Marcus Sperling ${ }^{a}$ and Harold C. Steinacker ${ }^{b}$ \\ ${ }^{a}$ Yau Mathematical Sciences Center, Tsinghua University, \\ Haidian District, Beijing, 100084, China \\ ${ }^{b}$ Faculty of Physics, University of Vienna, \\ Boltzmanngasse 5, A-1090 Vienna, Austria \\ E-mail: marcus.sperling@univie.ac.at, harold.steinacker@univie.ac.at
}

ABSTRACT: We discuss a $(3+1)$-dimensional covariant quantum space-time describing a FLRW cosmology with Big Bounce, obtained by a projection of the fuzzy hyperboloid $H_{n}^{4}$. This provides a background solution of the IKKT matrix model with mass term. We characterize the bosonic fluctuation spectrum, which consists of a tower of higher-spin modes, truncated at $n$. The modes are organized in terms of an underlying $\mathrm{SO}(4,2)$ structure group, which is broken to the $\mathrm{SO}(3,1)$ isometry of the background. The resulting higher-spin gauge theory includes all degrees of freedom required for gravity, and should be well suited for quantization. All modes propagate with the same speed of light, even though local boost invariance is not manifest. The propagating metric perturbation modes comprise those of a massless graviton, as well as a scalar mode. Gauge invariance allows to obtain the analog of the linearized Einstein-Hilbert action, which is expected to be induced upon quantization.

KEywords: M(atrix) Theories, Models of Quantum Gravity, Non-Commutative Geometry, Higher Spin Gravity

ARXIV EPRINT: 1901.03522 


\section{Contents}

1 Introduction 1

2 Geometric preliminaries 3

2.1 Classical hyperboloid $H^{4}$ from $\mathbb{C} P^{1,2} \quad 3$

2.2 The fuzzy hyperboloid $H_{n}^{4} \quad 6$

2.3 Projected quantum space-time $\mathcal{M}_{n}^{3,1}$ from $H_{n}^{4} \quad 9$

2.4 Semi-classical structure of $\mathcal{M}^{3,1} \quad 9$

2.5 Metric properties of $\mathcal{M}^{3,1} \quad 13$

3 Wavefunctions, higher-spin modes and covariance $\quad \mathbf{1 5}$

$\begin{array}{lll}3.1 & \text { Spin Casimir } & 16\end{array}$

$\begin{array}{lll}3.2 & \text { Space-like gauge and } \mathrm{SO}(3,1) \text {-covariant tensor fields } & 17\end{array}$

$\begin{array}{lll}3.3 & \mathrm{SO}(3,1) \text { intertwiners and sub-structure } & 18\end{array}$

$3.4 \mathrm{SO}(4,1)$-covariant gauge and tensor fields on $H^{4}$

4 Matrix model and quantum space-time solutions $\quad 24$

5 Fluctuations and higher-spin gauge theory $\quad \mathbf{2 5}$

$\begin{array}{ll}5.1 & \text { Mode expansion and ansatz } \\ 5.2 & 26\end{array}$

$\begin{array}{ll}5.2 \text { Group-theoretical considerations } & 27\end{array}$

$\begin{array}{lll}5.3 & \mathcal{D}^{2} \text { eigenvalues } & 29\end{array}$

5.4 Gauge fixing and physical Hilbert space $\quad 31$

5.5 Inner products and quadratic action 34

6 Metric and gravitons on $\mathcal{M}^{3,1} \quad 35$

$\begin{array}{lll}6.1 & \text { Linearized curvature-like tensors } & 39\end{array}$

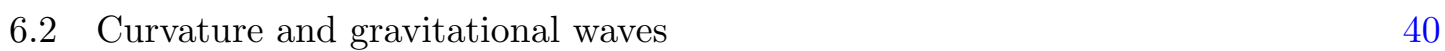

6.3 Linearized Einstein-Hilbert-like action 42

6.4 Discussion 44

$\begin{array}{lll}7 & \text { Conclusion and outlook } & 46\end{array}$

$\begin{array}{ll}\text { A Mathematical supplements and details } & 48\end{array}$

A.1 Derivation of the Einstein-Hilbert-like action 48

$\begin{array}{lll}\text { A.2 Conservation law } & 51\end{array}$

A.3 Time-like mode $\quad 52$

A.4 Group theory and useful identities 53

$\begin{array}{ll}\text { A.5 Consistency checks } & 56\end{array}$

$\begin{array}{lll}\text { A.6 Relations for } \square_{H} & 57\end{array}$ 


\section{Introduction}

The proper formulation of gravity as a quantum theory is a long-standing and controversial problem. Much of the difficulty is due to the fact that the gravitational constant is dimensionful, which means that gravity becomes strong at short scales, and arguably requires a quantum notion of space-time itself. It is thus plausible that the starting point of such a quantum theory could be very different from general relativity (GR), but GR should be approximately recovered at macroscopic scales, in the sense of an effective theory.

With this in mind, we choose the framework of matrix models of Yang-Mills type as a starting point. These models naturally describe dynamical noncommutative ("quantum") geometries, and they automatically lead to a gauge theory, which is crucial in Minkowski signature. Moreover, the kinetic term arises from a universal commutator structure, which encodes a universal metric [1]. To avoid the dangerous UV/IR mixing which typically arises on non-commutative spaces [2], one is led to the maximally supersymmetric IKKT or IIB model [3], where UV/IR mixing is sufficiently mild. ${ }^{1}$ This model can be viewed as a constructive definition of IIB string theory, and it exhibits a rich set of brane-type solutions, such as [7-12] to mention only a few. Quite remarkably, recent numerical simulations of the model [12-15] provide evidence that a $3+1$-dimensional structure indeed arises at the non-perturbative level.

With this motivation, our objective is to find a suitable background space-time solution of the IKKT matrix model, which leads to the desired low-energy physics including gravity. There has been considerable effort along these lines including [1, 16-19], and [20-26] in a similar spirit. One of the problems which arises on simple non-commutative geometries is that they typically carry some Lorentz-breaking structure, which is well hidden classically, but tends to show up in the loop contributions. That problem can be avoided on covariant quantum spaces, such as fuzzy $S_{N}^{4}, H_{n}^{4}$, and similar spaces [27-35]. This class of spaces exhibits a rich extra structure, which typically leads to a higher-spin gauge theory [36-38].

In this paper, we focus on an interesting candidate for such a covariant background, given by the quantized or fuzzy cosmological space-time $\mathcal{M}_{n}^{3,1}$ recently found in [39]. This (3+1)-dimensional space is based on the 4-dimensional fuzzy hyperboloid $H_{n}^{4}$ [40], which upon a projection acquires a semi-classical Friedmann-Lemaître-Robertson-Walker (FLRW) geometry describing an expanding universe with initial singularity, or rather a Big Bounce. In particular, we study the linearized fluctuation spectrum and the effective gauge theory arising on this $\mathcal{M}_{n}^{3,1}$, which is a solution of the matrix model with a mass term. For previous work on FLRW solutions in the context of higher spin gravity we refer to $[41,42]$.

One important feature of $\mathcal{M}_{n}^{3,1}$ is that it admits a global $\mathrm{SO}(3,1)$ symmetry, while local Lorentz invariance is not manifest. This $\mathrm{SO}(3,1)$ symmetry is the isometry group of the space-like hyperbolas $H_{t}^{3}$ of the cosmological space-time with $k=-1$. It comprises spacelike translations and local $\mathrm{SO}(3)$ rotations, and the latter can be viewed as a manifest

\footnotetext{
${ }^{1} \mathrm{UV} / \mathrm{IR}$ mixing leads to 10-dimensional "bulk" supergravity in the Type IIB model [3-6]. However, this has nothing to do with the mechanism under consideration here, which arises on a 4-dimensional brane, where the bulk supergravity only induces a short-range $r^{-8}$ interaction.
} 
part of Lorentz invariance. However, there is no boost invariance, because the cosmic background defines an invariant time-like vector field. Moreover, the model enjoys a large gauge invariance, which can be viewed as a truncated higher-spin algebra associated to $\mathfrak{s o}(4,1)[38,43]$, acting in an intricate way. These symmetries protect the theory from becoming pathological, and almost enforce local Lorentz invariance. For example, it turns out that the propagation of all physical higher-spin modes is governed by a single effective metric, as it should be, and we expect that the resulting physics will largely respect local Lorentz invariance. Thus the breaking of (local) Lorentz-invariance seems to be well hidden in the unphysical fluctuations, as far as we can see. Nevertheless, some breaking of Lorentz invariance is bound to show up somewhere.

The present $\mathcal{M}_{n}^{3,1}$ solution is somewhat different from (and in a sense dual to) the solution recently found in $[39,44]$. The present realization is preferred, because it allows to systematically organize and study the (linearized) fluctuation spectrum. This involves organizing the fluctuations into towers of higher-spin modes, finding the on-shell modes and their spectrum, and identifying among them the metric fluctuation modes. All these are non-trivial tasks which requires some technology. Fortunately, due to the close relation with the Euclidean case of $H_{n}^{4}$ and its enhanced $\mathrm{SO}(4,1)$ symmetry, we can use many of the results obtained in [40], and largely solve these problems. That $\mathrm{SO}(4,1)$ is in fact part of an $\mathrm{SO}(4,2)$ structure group, which is underlying the construction of $H_{n}^{4}$ as a coadjoint orbit. In particular (and in contrast to previous attempts), all physical metric degrees of freedom are obtained from the linearized fluctuation modes, as well as three of the usual four diffeomorphism modes, which arise as pure gauge modes of the higher-spin algebra. The reduction to three diffeomorphism modes reflects the presence of an invariant volume form on the noncommutative space.

Since GR is not a Yang-Mills theory, one would not expect to obtain the Einstein equations directly from the matrix model. It is therefore very remarkable that we do indeed obtain the linearized vacuum Einstein equations directly from the matrix model. More precisely, the classical action leads to the two propagating Ricci-flat graviton modes as in linearized GR, ${ }^{2}$ as well as a scalar metric mode whose significance is not yet clear. However to obtain the (linearized) Einstein equations in the presence of matter, quantum effects in the matrix model are presumably required. Here the higher-spin symmetry is extremely useful, because it strongly restricts the possible terms in the quantum effective action. Using this gauge invariance, we identify ${ }^{3}$ the analog of the linearized Einstein-Hilbert action, which is expected to be induced upon quantization [46]. Moreover, there seems to be no analog of the cosmological constant term, which is replaced by the Yang-Mills action. This suggests that the cosmological constant problem may not arise in this approach.

Due to the Yang-Mills structure of the underlying (matrix) model, the gauge-fixing procedure is straightforward, and the model should provide a well-defined quantum theory.

\footnotetext{
${ }^{2}$ This also works for $\mathbb{R}_{\theta}^{4}[1,20,22]$, but its extension to full gravity seems problematic, notably due to more serious Lorentz violations. There is in fact a way to formally obtain the full vacuum Einstein equations [21], which however leads to other issues.

${ }^{3}$ This would not be possible on simple noncommutative spaces such as $\mathbb{R}_{n}^{4}$, where extra terms are possible which are forbidden here [45]. This is very important for obtaining a realistic physics.
} 
There are only finitely many degrees of freedom per volume due to the intrinsic UV cutoff of the underlying noncommutative space-time, and the maximal supersymmetry of the model (broken by the soft mass term) will strongly restrict possible non-local quantum effects due to UV/IR mixing. Since Ricci-flat deformations are already solutions of the bare action and all metric modes of gravity are present, the model is an excellent candidate for realizing gravity in a quantum theory of space-time and matter.

As an extra bonus, the background under consideration provides a rather attractive cosmological scenario featuring a Big Bounce and an asymptotically coasting late-time evolution. It is fascinating that a reasonable cosmological evolution is obtained without any fine-tuning, and even without requiring the presence of matter. If the resulting gravity at intermediate length scales turns out to be viable, this would be a significant advantage over more conventional approaches, which typically require a delicate balance of various matter and energy constituents of the universe.

The outline of the paper is as follows: We start in section 2 by reviewing the classical geometry of $\mathbb{C} P^{1,2}$, which is understood either as coadjoint orbit or as total space of an $S^{2}$-bundle over $H^{4}$ or $H^{2,2}$. Its quantization leading to fuzzy $H_{n}^{4}$ as well as the projection to fuzzy $\mathcal{M}_{n}^{3,1}$ are then detailed. We study the algebra of functions in section 3 by means of spin Casimirs and various intertwiners. It will turn out that two descriptions are available: either divergence-free symmetric traceless tensors on $H^{4}$ or space-like symmetric traceless tensors on $\mathcal{M}^{3,1}$. As shown in section 4, the IKKT matrix model allows three kinds of solutions from various embeddings of the $\mathfrak{s o}(4,2)$ generators. Focusing on the momentum solution as classical background, we investigate the fluctuation modes and the resulting higher-spin gauge theory in section 5. We pay attention to gauge-fixing questions as well as the inner products and kinetic terms. All these considerations prepare the stage for section 6 , in which we address the definition of gravition modes as well as the construction of an gauge-invariant quadratic action. We elaborate the properties of this linearized Einstein-Hilbert-like action in detail. Finally we conclude and summarize in section 7 . The appendix A provides details and proofs of some elaborate calculations that were omitted in the main text. To make the paper self-contained and to facilitate follow-up work, we include all the required background and details.

\section{Geometric preliminaries}

\subsection{Classical hyperboloid $H^{4}$ from $\mathbb{C} P^{1,2}$}

We start with a discussion of the classical geometry underlying fuzzy $H_{n}^{4}$ which is $\mathbb{C} P^{1,2}$, viewed as an $S^{2}$ bundle over the 4 -hyperboloid $H^{4}$. More precisely, $\mathbb{C} P^{1,2}$ is an $\mathrm{SO}(4,1)$ equivariant bundle over $H^{4}$, meaning that there exists an $\mathrm{SO}(4,1)$ action on both the total space and the base space that are compatible with the bundle projection [47, Def. 1.5]. The local stabilizer group $\mathrm{SO}(4)$ acts non-trivially on the $S^{2}$ fiber such that the internal excitations become higher-spin fields on $H^{4}$. In addition, $\mathbb{C} P^{1,2}$ is a coadjoint orbit of $\mathrm{SO}(4,2)$, which provides extra geometrical structure and, naturally, leads to a quantization in terms of fuzzy $H_{n}^{4}$. The construction shares some similarities with twistor constructions for Minkowski space. 
Conventions. Latin letters $a, b, c, \ldots$ will typically imply the natural representation of $\mathrm{SO}(4,1)$, and Greek letters $\mu, \nu, \gamma, \ldots$ the $\mathrm{SO}(3,1)$ version. We will generally raise and lower indices with the $\mathrm{SO}(4,1)$-invariant tensor $\eta^{a b}$ or its $\mathrm{SO}(3,1)$ version $\eta^{\mu \nu}$, unless otherwise stated. Moreover, Einstein summation convention is adopted, i.e. repeated indices are summed over.

$\mathbb{C} P^{1,2}$ as $\mathrm{SO}(4,1)$-equivariant bundle over the hyperboloid $\boldsymbol{H}^{4}$. Let $\psi \in \mathbb{C}^{4}$ be a spinor of $\mathfrak{s o}(4,1)$ with normalization $\bar{\psi} \psi=1$, and consider the following Hopf map:

$$
\begin{aligned}
x^{a}: \quad \mathbb{C} P^{1,2} & \rightarrow H^{4} \subset \mathbb{R}^{1,4} \\
\psi & \mapsto x^{a}=\frac{r}{2} \bar{\psi} \gamma^{a} \psi, \quad a=0,1,2,3,4
\end{aligned}
$$

noting that the phase of $\psi$ drops out. Here $\mathbb{C} P^{1,2}$ is defined as space of unit spinors $\bar{\psi} \psi=1$ modulo $\mathrm{U}(1)$, and $r$ introduces a length scale. One can verify that

$$
\sum_{a, b=0}^{4} \eta_{a b} x^{a} x^{b}=-\frac{r^{2}}{4}=:-R^{2}
$$

implying that the right-hand-side is indeed in $H^{4}$. The map (2.1) is a non-compact version of the Hopf map $\mathbb{C} P^{3} \rightarrow S^{4}$, and, moreover, it is an $\mathrm{SO}(4,1)$-intertwiner provided the $x^{a}$ transform as $\mathrm{SO}(4,1)$ vectors. The fiber can be seen to be $S^{2}$ such that $\mathbb{C} P^{1,2}$ is an equivariant $S^{2}$-bundle over $H^{4}$; for more details, we refer to [40]. Note that the metric on the $H^{4} \subset \mathbb{R}^{1,4}$ is Euclidean, despite the $\mathrm{SO}(4,1)$ metric on target space. This is obvious at the point $\xi=(R, 0,0,0,0)$, where the tangent space is $\mathbb{R}_{1234}^{4}$.

$\mathbf{S O}(4,2)$ formulation and embedding functions. As mentioned above, $\mathbb{C} P^{1,2}$ is a 6 -dimensional coadjoint orbit of $\mathrm{SU}(2,2)$, given by

$$
\mathbb{C} P^{1,2} \cong\left\{U^{-1} Z U, \quad U \in \mathrm{SU}(2,2)\right\} \hookrightarrow \mathfrak{s u}(2,2)
$$

where $Z$ is a rank one $4 \times 4$ matrix defined by

$$
Z=\psi \bar{\psi}, \quad Z^{2}=Z, \quad \operatorname{tr}(Z)=1, \quad Z^{\dagger}=\gamma^{0} Z \gamma^{0-1}
$$

The embedding (2.3) is described by the embedding functions

$$
\begin{aligned}
m^{a b} & =\operatorname{tr}\left(Z \Sigma^{a b}\right)=\bar{\psi} \Sigma^{a b} \psi=\left(m^{a b}\right)^{*}, \\
x^{a} & =r m^{a 5}, \quad a, b=0, \ldots, 4
\end{aligned}
$$

noting that $\frac{1}{2} \gamma^{a}=\Sigma^{a 5}$. Upon restricting to $\mathfrak{s o}(4,1) \subset \mathfrak{s o}(4,2) \cong \mathfrak{s u}(2,2)$, we recover $(2.1)$, which reflects the transitivity of the $\mathrm{SO}(4,1)$ action on $\mathbb{C} P^{1,2}$. The last equation in (2.5) encodes the Hopf map, which will generalize to the non-commutative case. The $\mathrm{SO}(4,2)$ structure is often useful, but it does not respect the projection to $H^{4}$. 
We briefly recall some of the resulting algebraic relations, which are derived and discussed in [40]. One can compute the invariant functions

$$
\begin{aligned}
\sum_{0 \leq a<b \leq 4} m^{a b} m_{a b} & =\frac{1}{2}, \\
\sum_{0 \leq a<b \leq 5} m^{a b} m_{a b} & =\frac{3}{4} .
\end{aligned}
$$

Here, the indices are raised and lowered with $\eta_{a b}=\operatorname{diag}(-1,1,1,1,1,-1)$. Recalling (2.5), we recover

$$
x_{a} x^{a} \equiv \sum_{a=0}^{4} x_{a} x^{a}=-\frac{r^{2}}{4}=-R^{2} .
$$

It is remarkable that the $\mathrm{SO}(4,1)$-invariant $x^{a} x_{a}$ is constant on the $\mathrm{SO}(4,2)$ orbit $\mathbb{C} P^{1,2}$. Similarly, the relation $Z^{2}=Z$ of $(2.4)$ entails the $\mathrm{SO}(4,2)$ identity

$$
\sum_{c, d=0}^{5} \eta_{c d} m^{a c} m^{b d}=\frac{1}{4} \eta^{a b}, \quad a, b=0, \ldots, 5,
$$

which reduces to the $\mathrm{SO}(4,1)$ relation

$$
\eta_{c d} m^{a c} m^{b d}-r^{-2} x^{a} x^{b}=\frac{1}{4} \eta^{a b}, \quad a, b=0, \ldots, 4 .
$$

In particular, (2.10) implies that $m^{a b}$ is orthogonal to $x^{a}$, i.e.

$$
x_{a} m^{a b}=0, \quad b=0, \ldots, 4 .
$$

Furthermore, the following $\mathrm{SO}(4,2)$ identity holds

$$
\epsilon_{a b c d e f} m^{a b} m^{c d}=4 m_{e f},
$$

which reduced to $\mathrm{SO}(4,1)$ implies

$$
\epsilon_{a b c d e} m^{a b} m^{c d}=-\frac{4}{r} x_{e}, \quad e=0, \ldots, 4 .
$$

Finally, there exists a self-duality relation

$$
\epsilon_{a b c d e} m^{a b} x^{c}=m_{d e} .
$$

Thus $m^{a b}$ is a tangential self-dual rank 2 tensor on $H^{4}$, in complete analogy to the fuzzy 4 -sphere $S_{N}^{4}$ discussed in [35]. One can thus express $m^{a b}$ in terms of the $\mathrm{SO}(4)$ t'Hooft symbols

$$
m^{\mu \nu}=\eta_{\mu \nu}^{i} J_{i}, \quad J_{i} J^{i}=1
$$

where $J_{i}, i=1,2,3$ describes the internal $S^{2}$. This exhibits the structure of $\mathbb{C} P^{1,2}$ is an $\mathrm{SO}(4,1)$-equivariant bundle over $H^{4}$. The $S^{2}$ fiber is generated by the local $\mathrm{SU}(2)_{L}$, while $\mathrm{SU}(2)_{R}$ acts trivially. 
Projection to $\boldsymbol{H}^{\mathbf{2 , 2}}$. Alternatively, the $\mathrm{SO}(4,2)$ homogeneous space $\mathbb{C} P^{1,2}$ can be viewed as $S^{2}$-bundle over $H^{2,2}$, which arises from the $\mathrm{SO}(3,2)$-equivariant Hopf map

$$
\begin{aligned}
t^{a}: \quad \mathbb{C} P^{1,2} & \rightarrow H^{2,2} \subset \mathbb{R}^{2,3} \\
\psi & \mapsto \frac{1}{R} \bar{\psi} \Sigma^{a 4} \psi=\frac{1}{R} m^{a 4}, \quad a=0,1,2,3,5 .
\end{aligned}
$$

Now, the coordinate functions $t^{a}$ define a hyperboloid $H^{2,2} \subset \mathbb{R}^{3,2}$ with intrinsic signature $(+,+,-,-)$. Using analogous identities as before, we obtain the constraints

$$
\begin{aligned}
\tilde{\eta}_{a b} t^{a} t^{b} & =r^{-2}, & \tilde{\eta}_{a b} & =\operatorname{diag}(-1,1,1,1,-1), \\
t_{a} x^{a} & =0 \equiv t_{\mu} x^{\mu}, & \mu & =0,1,2,3 .
\end{aligned}
$$

The last relation in (2.17) follows from (2.9), noting that $t^{4} \equiv 0$.

SO(3,1)-invariant projection and Minkowski signature. Neither of the spaces $H^{4}$ or $H^{2,2}$ considered so far has Minkowski signature. Fortunately, space-times with Minkowski signature can be obtained by $\mathrm{SO}(3,1)$-covariant projections of the above hyperboloids. Explicitly, consider the projections [39, 40]

$$
\begin{array}{rlrl}
\Pi_{x}: \quad \mathbb{C} P^{1,2} & \rightarrow \mathbb{R}_{x}^{1,3} & \\
m & \mapsto x^{\mu}=r m^{\mu 5}, & & \mu=0,1,2,3, \\
\Pi_{t}: \quad \mathbb{C P}^{1,2} & \rightarrow \mathbb{R}_{t}^{1,3} & & \\
m & \mapsto t^{\mu}=R^{-1} m^{\mu 4}, & \mu=0,1,2,3,
\end{array}
$$

which respect $\mathrm{SO}(3,1)$. In section 2.3 , the image $\mathcal{M}^{3,1} \subset \mathbb{R}_{x}^{3,1}$ of $\Pi_{x}$ will serve as cosmological FLRW space-time with $k=-1$ and initial singularity due to $x^{0}>0$. The local stabilizer $\mathrm{SO}(3)$ acts on the $S^{2}$ fiber. In contrast, the image of $\Pi_{t}$ covers the space-like region of $\mathbb{R}_{t}^{3,1}$ with norm $t_{\mu} t^{\mu} \geq r^{2}$, and the local stabilizer $\mathrm{SO}(2,1)$ acts on the $H^{1,1}$ fiber. This does not seem to give an interesting space-time, but it will be related to momentum space.

\subsection{The fuzzy hyperboloid $H_{n}^{4}$}

Now we consider the quantization of the geometry discussed above. This is based on the fuzzy 4-hyperboloid $H_{n}^{4}$, which is a quantization of the $S^{2}$-bundle $\mathbb{C} P^{1,2} \rightarrow H^{4}$ with the canonical Poisson structure on $\mathbb{C} P^{1,2}$. Fuzzy $H_{n}^{4}$ was introduced in [48] and further developed in [44], and we briefly recapitulate the main results. As for any coadjoint orbit, the canonical quantization of $\mathbb{C} P^{1,2}$ proceeds in terms of the operator algebra $\operatorname{End}\left(\mathcal{H}_{n}\right)$ where $\mathcal{H}_{n}$ is a suitable unitary irreducible representation (irrep) of $\mathrm{SU}(2,2) \cong \mathrm{SO}(4,2)$. The representation is chosen such that the Lie algebra generators $\mathcal{M}^{a b} \in \operatorname{End}\left(\mathcal{H}_{n}\right)$ generate a quantized algebra of functions, interpreted as fuzzy $\mathbb{C} P_{n}^{1,2}$. The $\mathcal{M}^{a b}$ are naturally viewed as quantized coordinate functions $m^{a b}$ on $\mathfrak{s o}(4,2)$. Analogously to the Hopf map (2.1), fuzzy $H_{n}^{4}$ is defined via a projection of $\mathbb{C} P_{n}^{1,2}$ that is generated by Hermitian operators $X^{a} \sim x^{a}$, transforming as vectors under $\mathrm{SO}(4,1) \subset \mathrm{SO}(4,2)$. 
To define fuzzy $H_{n}^{4}$ explicitly, let $\eta_{a b}=\operatorname{diag}(-1,1,1,1,1,-1)$ be the invariant metric of $\mathrm{SO}(4,2)$, and $\mathcal{M}_{a b}$ be Hermitian generators of $\mathfrak{s o}(4,2)$ which satisfy

$$
\left[\mathcal{M}_{a b}, \mathcal{M}_{c d}\right]=\mathrm{i}\left(\eta_{a c} \mathcal{M}_{b d}-\eta_{a d} \mathcal{M}_{b c}-\eta_{b c} \mathcal{M}_{a d}+\eta_{b d} \mathcal{M}_{a c}\right)
$$

We choose a particular type of (discrete series) positive-energy unitary irreps $\mathcal{H}_{n}$ known as minireps or doubletons $[49,50]$, which, remarkably, remain irreducible under the restriction to $\mathrm{SO}(4,1) \subset \mathrm{SO}(4,2)$. This follows from the minimal oscillator construction of $\mathcal{H}_{n}$, where all $\mathrm{SO}(4,2)$ weight multiplicities are at most one, cf. [49, 51, 52]. Strictly speaking there are two versions $\mathcal{H}_{n}^{L}$ or $\mathcal{H}_{n}^{R}$ with opposite chirality, but this distinction is irrelevant in the present paper and can therefore be dropped.

The doubletons $\mathcal{H}_{n}$ have positive discrete spectrum

$$
\operatorname{spec}\left(\mathcal{M}^{05}\right)=\left\{E_{0}, E_{0}+1, \ldots\right\}, \quad E_{0}=1+\frac{n}{2}
$$

where the eigenspace with lowest eigenvalue of $\mathcal{M}^{05}$ is an $(n+1)$-dimensional irreducible representation of either $\mathrm{SU}(2)_{L}$ or $\mathrm{SU}(2)_{R}$. Then the Hermitian generators

$$
\begin{aligned}
X^{a} & :=r \mathcal{M}^{a 5}, & & a=0,1,2,3,4, \\
T^{a} & :=R^{-1} \mathcal{M}^{a 4}, & & a=0,1,2,3,5
\end{aligned}
$$

satisfy

$$
\begin{aligned}
& {\left[X^{a}, X^{b}\right]=-\mathrm{i} r^{2} \mathcal{M}^{a b}=: \mathrm{i} \Theta^{a b}} \\
& {\left[T^{\mu}, X^{\nu}\right]=\frac{r}{R}\left[\mathcal{M}^{\mu 4}, \mathcal{M}^{\nu 5}\right]=\mathrm{i} \frac{1}{R} \eta^{\mu \nu} X^{4}} \\
& {\left[T^{\mu}, X^{4}\right]=\frac{r}{R}\left[\mathcal{M}^{\mu 4}, \mathcal{M}^{45}\right]=-\mathrm{i} \frac{1}{R} X^{\mu}} \\
& {\left[T^{5}, X^{\nu}\right]=\frac{r}{R}\left[\mathcal{M}^{54}, \mathcal{M}^{\nu 5}\right]=-\mathrm{i} r T^{\nu}} \\
& {\left[T^{\mu}, T^{\nu}\right]=\frac{\mathrm{i}}{R^{2}} \mathcal{M}^{\mu \nu}=-\frac{\mathrm{i}}{r^{2} R^{2}} \Theta^{\mu \nu}}
\end{aligned}
$$

The $X^{a}$ transform as $\mathrm{SO}(4,1)$ vectors, i.e.

$$
\begin{aligned}
{\left[\mathcal{M}_{a b}, \mathcal{M}_{c d}\right] } & =\mathrm{i}\left(\eta_{a c} \mathcal{M}_{b d}-\eta_{a d} \mathcal{M}_{b c}-\eta_{b c} \mathcal{M}_{a d}+\eta_{b d} \mathcal{M}_{a c}\right) \\
{\left[\mathcal{M}_{a b}, X_{c}\right] } & =\mathrm{i}\left(\eta_{a c} X_{b}-\eta_{b c} X_{a}\right)
\end{aligned}
$$

for $a, b, c, d \in\{0, \ldots, 4\}$. Since the restriction $\mathrm{SO}(4,1) \subset \mathrm{SO}(4,2)$ is irreducible, it follows that the $X^{a}$ live on a hyperboloid,

$$
\sum_{a, b=0,1,2,3,4} \eta_{a b} X^{a} X^{b}=\sum_{a=1}^{4} X^{a} X^{a}-X^{0} X^{0}=-R^{2} \mathbb{1}
$$

with radius [40]

$$
R^{2}=\frac{r^{2}}{4}\left(n^{2}-4\right)
$$


and

$$
\sum_{a, b=0,1,2,3,4,5} \eta_{a b} \mathcal{M}^{a c} \mathcal{M}^{b d}+(c \leftrightarrow d)=\frac{1}{2}\left(n^{2}-4\right) \eta^{c d}=2 \frac{R^{2}}{r^{2}} \eta^{c d} .
$$

Since $X^{0}=r \mathcal{M}^{05}>0$ has positive spectrum, this describes a one-sided hyperboloid in $\mathbb{R}^{1,4}$, denoted as $H_{n}^{4}$. Similarly, the $T^{a}$, for $a=0,1,2,3,5$ transform as $\mathrm{SO}(3,2)$ vectors and satisfy

$$
\sum_{a, b=0,1,2,3,5} \eta_{a b} T^{a} T^{b}=\sum_{i=1}^{3} T^{i} T^{i}-T^{5} T^{5}-T^{0} T^{0}=\frac{1}{r^{2}} \mathbb{1}
$$

and the $T^{a}$ generate a hyperboloid $H^{2,2}$ with signature $(--++)$. This reflects the fact that the reduction to $\mathrm{SO}(3,2) \subset \mathrm{SO}(4,2)$ of $\mathcal{H}_{n}$ is also irreducible. Further identities include

$$
\begin{aligned}
\sum_{b=0}^{4} \mathcal{M}^{a b} X_{b}+X_{b} \mathcal{M}^{a b} & =0, & & a=0,1,2,3,4, \\
\sum_{b=0,1,2,3,5} \mathcal{M}^{a b} T_{b}+T_{b} \mathcal{M}^{a b} & =0, & & a=0,1,2,3,5 .
\end{aligned}
$$

Analogous to fuzzy $S_{N}^{4}$, the semi-classical geometry underlying $H_{n}^{4}$ and $H^{2,2}$ is $\mathbb{C} P^{1,2}$ [48], which is an $S^{2}$-bundle over $H^{4}$ or $H^{2,2}$ carrying a canonical symplectic structure, respectively. In the fuzzy case, the typical fiber becomes a fuzzy 2-sphere $S_{n}^{2}$. However, for most parts of this paper, we work again in the semi-classical limit. It is important to note that the induced metric on the hyperboloid $H^{4} \subset \mathbb{R}^{1,4}$ is Euclidean, despite the $\operatorname{SO}(4,1)$ isometry. This can be seen at the point $x=(R, 0,0,0,0)$, where the tangent space is $\mathbb{R}_{1234}^{4}$.

Coherent states and quantization map. Since $\mathcal{H}_{n}$ are lowest weight representations, there is a natural definition of coherent states on $\mathbb{C} P_{n}^{1,2}$, which are defined to be the $\operatorname{SO}(4,2)$ orbits of the lowest weight state in $\mathcal{H}_{n}$. The ambiguity in the choice of the group element $g \in \mathrm{SO}(4,2)$ leads to a $\mathrm{U}(1)$ phase ambiguity, so that the coherent states $\{|z\rangle\}$ furnish a $\mathrm{U}(1)$-bundle over $\mathbb{C} P^{1,2}$, or a $\mathrm{U}(2)$-bundle over $H^{4}$. For more details on coherent states we refer to [53-57].

Given these coherent states, there is a natural $\mathrm{SO}(4,2)$-equivariant quantization map from the classical space of functions on $\mathbb{C} P^{1,2}$ to the fuzzy functions $\operatorname{End}\left(\mathcal{H}_{n}\right)$ :

$$
\begin{aligned}
\mathcal{Q}: \quad \mathcal{C}\left(\mathbb{C} P^{1,2}\right) & \rightarrow \operatorname{End}\left(\mathcal{H}_{n}\right) \\
\phi(z) & \mapsto \hat{\phi}=\int_{\mathbb{C} P^{1,2}} d \mu \phi(z)|z\rangle\langle z| .
\end{aligned}
$$

Up to a cutoff, this map is one-to-one, ${ }^{4}$ and the inverse is given by the symbol

$$
\hat{\phi} \in \operatorname{End}\left(\mathcal{H}_{n}\right) \mapsto\langle z|\hat{\phi}| z\rangle=\phi(z) \in \mathcal{C}\left(\mathbb{C} P^{1,2}\right),
$$

\footnotetext{
${ }^{4}$ To prove this, one can show that the decomposition into irreps is the same, cf. [58].
} 
up to mode-dependent normalization. Since $\mathcal{Q}$ respects $\mathrm{SO}(4,2)$, the generators act as

$$
\left[\mathcal{M}^{a b}, \mathcal{Q}(\phi)\right]=\mathcal{Q}\left(\left\{m^{a b}, \phi(z)\right\}\right)
$$

since $\left\{m^{a b}, \cdot\right\}$ implements the $\mathrm{SO}(4,2)$ action on $\mathcal{C}\left(\mathbb{C} P^{1,2}\right)$. In particular, the Laplacian operator(s) are respected too, e.g.

$$
\square \mathcal{Q}(\phi)=\mathcal{Q}\left(\square_{c l} \phi\right) \quad \text { with } \quad \square=\left[T^{\mu},\left[T_{\mu}, \cdot\right]\right], \quad \square_{c l}=-\left\{t^{\mu},\left\{t_{\mu}, \cdot\right\}\right\},
$$

and similarly for other operators based on $\mathrm{SO}(4,2)$.

\subsection{Projected quantum space-time $\mathcal{M}_{n}^{3,1}$ from $H_{n}^{4}$}

In this paper, we study the $\mathrm{SO}(3,1)$-invariant fuzzy space-time generated by the coordinates $X^{\mu}$ for $\mu=0,1,2,3$. As introduced in [39], this space-time can be viewed as projection $\Pi_{x}$ of $H^{4}$ onto the 0123 plane, and describes a homogeneous and isotropic quantized cosmological space-time ${ }^{5} \mathcal{M}_{n}^{1,3}$. The relations (2.25) and (2.28) lead to

$$
\begin{aligned}
\eta_{\mu \nu} X^{\mu} X^{\nu} & =-R^{2}-X^{4} X^{4}, \\
\eta_{\mu \nu} T^{\mu} T^{\nu} & =\frac{1}{r^{2}}+\frac{1}{r^{2} R^{2}} X^{4} X^{4}, \quad \mu, \nu=0, \ldots, 3
\end{aligned}
$$

recalling that $r R T^{5}=-X^{4}$. Finally, the constraint (2.27) yields

$$
0=X_{\mu} T^{\mu}+T^{\mu} X_{\mu} .
$$

The cosmological $\mathcal{M}^{3,1}$ background embedded by $X^{\mu} \sim x^{\mu}$ is covariant under $\operatorname{SO}(3,1)$, which is the symmetry respected by $\square$. The $T^{\mu}$ will serve as momentum generators on $\mathcal{M}_{n}^{1,3}$, even though they could considered as defining a quantum space in their own right [39].

\subsection{Semi-classical structure of $\mathcal{M}^{3,1}$}

Now we discuss the semi-classical limit $\mathcal{M}^{3,1}$ and its associated bundle structure in more detail. The basic object is the space $\mathcal{C}$ of functions on $\mathbb{C} P^{2,1}$, which can be viewed as equivariant bundle over $\mathcal{M}^{3,1}$ or $H^{4}$, i.e. as module over the algebra $\mathcal{C}^{0} \subset \mathcal{C}$ of functions on the base. The $\mathrm{SO}(4,1)$-covariant functions $\theta^{a b}$ and $x^{a}$ on $\mathbb{C} P^{2,1}$ over $\mathcal{M}^{3,1}$ separate as follows: the $x^{\mu}$ serve as coordinates on $\mathcal{M}^{3,1}$ and generate the algebra $\mathcal{C}^{0} \subset \mathcal{C}$ of functions ${ }^{6}$ on $\mathcal{M}^{3,1}$, whereas the $\theta^{\mu \nu}$ and $t^{\mu}$ generate the module $\mathcal{C}$ over $\mathcal{C}^{0}$. The constraints $(2.27)$ imply the following relations on the generators:

$$
\begin{aligned}
x_{\mu} x^{\mu} & =-R^{2}-x_{4}^{2}=-R^{2} \cosh ^{2}(\eta), \\
t_{\mu} t^{\mu} & =r^{-2} \cosh ^{2}(\eta), \\
t_{\mu} x^{\mu} & =0, \\
t_{\mu} \theta^{\mu \alpha} & =-\sinh (\eta) x^{\alpha}, \\
x_{\mu} \theta^{\mu \alpha} & =-r^{2} R^{2} \sinh (\eta) t^{\alpha}, \\
\eta_{\mu \nu} \theta^{\mu \alpha} \theta^{\nu \beta} & =R^{2} r^{2} \eta^{\alpha \beta}-R^{2} r^{4} t^{\alpha} t^{\beta}+r^{2} x^{\alpha} x^{\beta} \\
\theta^{\mu \nu} \theta_{\mu \nu} & =2 R^{2} r^{2}\left(2-\cosh ^{2}(\eta)\right)
\end{aligned}
$$

\footnotetext{
${ }^{5}$ We change notation from [39], where $Y^{1}$ was dropped instead of $Y^{4}$.

${ }^{6}$ Strictly speaking the $x^{\mu}$ cannot distinguish the two sheets of $\mathcal{M}^{3,1}$, which requires e.g. $x^{4}$.
} 
where $\mu, \alpha=0, \ldots, 3$. Here $\eta$ serves as a global time coordinate, and $x_{4}$ is a measure for the current size i.e. the curvature scale of the universe. From the radial constraint (2.8) on $H^{4}$ one deduces $\left\{x_{a} x^{a}, x^{\mu}\right\}=0$, which further implies

$$
0=\sum_{a=0}^{4} x_{a} m^{a \mu}=\sum_{\nu=0}^{3} x_{\nu} m^{\nu \mu}+x_{4} m^{4 \mu} .
$$

This establishes a relation between the momenta and the $t^{\mu}$,

$$
t^{\mu}=\frac{1}{R} m^{\mu 4}=-\frac{1}{R r^{2} x^{4}} x_{\nu} \theta^{\nu \mu} \stackrel{\xi}{=} \frac{1}{R r^{2}} \frac{1}{\tanh (\eta)} \theta^{0 \mu},
$$

where $\xi$ is a reference point on $H^{4}$ which can be chosen as

$$
\xi=\left(x^{0}, 0,0,0, x^{4}\right) \stackrel{\Pi}{\rightarrow}\left(x^{0}, 0,0,0\right), \quad x^{0}=R \cosh (\eta), \quad x^{4}=R \sinh (\eta)
$$

via $\mathrm{SO}(3,1)$-invariance. Furthermore, the self-duality constraint in $H_{n}^{4}[40]$

$$
\epsilon_{a b c d e} \theta^{a b} x^{c}=n r \theta_{d e}
$$

reduces to

$$
\begin{aligned}
& t^{i}=\frac{1}{R} m_{i 4}=\frac{1}{n R r^{3}} \epsilon_{a b c i 4} \theta^{a b} x^{c} \stackrel{\underline{\xi}}{=} \frac{1}{n r^{3}} \cosh (\eta) \epsilon^{i j k} \theta^{j k}, \\
& t^{0} \stackrel{\underline{\xi}}{=} 0
\end{aligned}
$$

where the last equation is simply a consequence of $x_{\mu} t^{\mu}=0$. Therefore $t^{\mu}$ describes a space-like $S^{2}$ with radius $r^{-2} \cosh ^{2}(\eta)$. As a remark, the form (2.41) only applies in the special $\mathfrak{s o}(3,1)$ adapted frame, and it is not generally covariant; of course on Minkowski manifolds, there is no notion of self-duality. Such compact extra dimensions which transform covariantly under $\mathrm{SO}(3,1)$ are possible only due to the partial breaking of Poincare covariance the present cosmological background.

Conversely, the relations (2.41) allow to express $\theta^{\mu \nu}$ in terms of the momenta $t^{\mu}$ as follows:

$$
\begin{aligned}
& \theta^{i j} \stackrel{\underline{\xi}}{=} \frac{n r^{3}}{2 \cosh (\eta)} \varepsilon^{i j k} t^{k}, \\
& \theta^{0 i} \stackrel{\underline{\xi}}{=} R r^{2} \tanh (\eta) t^{i},
\end{aligned}
$$

at the reference point $\xi$. By means of $R \sim \frac{1}{2} n r$ from $(2.26)$ and using $\mathrm{SO}(3,1)$ covariance, one can write (2.42) globally as follows:

$$
\begin{aligned}
& \theta^{\mu \nu}=c\left(x^{\mu} t^{\nu}-x^{\nu} t^{\mu}\right)+b \epsilon^{\mu \nu \alpha \beta} x_{\alpha} t_{\beta}=: r^{2} R \eta_{\alpha}^{\mu \nu}(x) t^{\alpha} \\
& \text { with } \quad c=r^{2} \frac{\sinh (\eta)}{\cosh ^{2}(\eta)} \quad \text { and } \quad b=\frac{n r^{3}}{2 R \cosh ^{2}(\eta)} .
\end{aligned}
$$

Hence $\eta_{\alpha}^{\mu \nu}(x)$ is a $\mathrm{SO}(3,1)$-invariant tensor field on $\mathcal{M}^{3,1}$, which is analogous of the t'Hooft symbols. Moreover, note that $\theta^{0 i} \gg \theta^{i j}$ for late times $\eta \gg 1$; this reflects the embedding of $H^{4} \subset \mathbb{R}^{4,1}$ which approaches the light cone at late times. Consequently, space is almost commutative, but space-time is not. The effects of non-commutativity will be further weakened due to the averaging on $S^{2}$. 
Poisson calculus. We can define a canonical "Poisson" connection on $\mathcal{C}$, viewed as module ${ }^{7}$ over the functions $\mathcal{C}^{0}$ on $\mathcal{M}^{3,1}$, by

$$
\nabla_{e_{\mu}}:=\left\{t_{\mu}, \cdot\right\}: \quad \mathcal{C} \rightarrow \mathcal{C}, \quad e_{\mu}=\frac{x^{4}}{R} \partial_{\mu}
$$

Here $e_{\mu}$ are vector fields on $\mathcal{M}^{3,1}$ defined in terms of the Cartesian coordinates $x^{\mu}$. Explicitly,

$$
\begin{aligned}
\nabla_{e_{\mu}} x^{\nu} & =\frac{x^{4}}{R} \delta_{\mu}^{\nu} \\
\nabla_{e_{\mu}} \theta^{\alpha \beta} & =r^{2}\left(-\delta_{\mu}^{\alpha} t^{\beta}+\delta_{\mu}^{\beta} t^{\alpha}\right), \\
\nabla_{e_{\mu}} t^{\alpha} & =-\frac{1}{r^{2} R^{2}} \eta_{\mu \nu} \theta^{\nu \alpha}
\end{aligned}
$$

Note that $\nabla$ defines an $\mathrm{SO}(3,1)$-equivariant connection on each $H^{3}$. However $\mathrm{SO}(3,1)$ invariance does not uniquely determine a connection on $\mathcal{M}^{3,1}$, since any conformal rescaling of $\eta^{\mu \nu}$ defines a different FLRW metric and, thus, a different connection on $\mathcal{M}^{3,1}$. Nevertheless, $\nabla$ is the unique connection that respects the symplectic form, as discussed below. This amounts to an extra structure on $\mathcal{M}^{3,1}$ which is not present in Riemannian geometry. We also note the following identity:

$$
\left\{\mathcal{M}^{\mu \nu}, \phi\right\}=-\left(x^{\mu} \partial^{\nu}-x^{\nu} \partial^{\mu}\right) \phi, \quad \phi \in \mathcal{C}^{0}
$$

Furthermore, (2.36) implies

$$
\partial_{\mu} x_{4}=-\frac{1}{x^{4}} x_{\mu} \quad \text { and } \quad \partial_{\mu} f(\eta)=-\frac{x_{\mu}}{R^{2}} \frac{1}{\sinh (\eta) \cosh (\eta)} \frac{\partial}{\partial \eta} f(\eta) .
$$

Integration and measure. As for any quantized coadjoint orbit, the trace on $\operatorname{End}\left(\mathcal{H}_{n}\right)$ corresponds to the integral over the underlying symplectic space, i.e.

$$
\operatorname{Tr} \mathcal{Q}(\phi)=\int_{\mathbb{C} P^{1,2}} \mathrm{~d} \Omega \phi=\int_{H^{4}} \rho_{H}[\phi]_{0}=\int_{\mathcal{M}^{4}} \rho_{M}[\phi]_{0}
$$

where $\mathrm{d} \Omega$ is the $\mathrm{SO}(4,2)$-invariant volume form on $\mathbb{C} P^{1,2}$ arising from the (Kirillov-Kostant) symplectic form. We aim to find the explicit form on $H^{4}$ and $\mathcal{M}^{3,1}$ in terms of the cosmological time $\eta$, which determines the $\mathrm{SO}(3,1)$-invariant space-like slice $H^{3} \subset H^{4} \subset \mathbb{R}^{4,1}$ via $x_{\mu} x^{\mu}=-R^{2} \cosh ^{2}(\eta)$ from (2.36). At the reference point $\xi, x_{0}=R \cosh (\eta)$ and $\mathrm{d} x_{0}=R \sinh \eta \mathrm{d} \eta$ such that the $\mathrm{SO}(4,1)$-invariant volume form on $H^{4}$ can be written as

$$
\rho_{H}=\frac{4}{L_{N C}^{4}} R \mathrm{~d} \eta \mathrm{d} \Omega_{3}=\frac{4}{L_{N C}^{4}} \frac{1}{\sinh (\eta)} \mathrm{d} x_{0} \ldots \mathrm{d} x_{3}
$$

\footnotetext{
${ }^{7}$ This means that $\mathcal{C}$ describes a vector bundle over $\mathcal{M}^{3,1}$. This connection respects the refined $\mathcal{C}^{s}$ modules, which are introduced in section 3.1. The construction could be generalized to the noncommutative case, but we restrict ourselves to the semi-classical geometry here for simplicity.
} 
where $\mathrm{d} \Omega_{3}$ is the induced volume form $H^{3}$. This can be pulled back to $\mathcal{M}^{3,1}$ using $\sinh (\eta)=$ $\frac{x_{4}}{R}$, which yields

$$
\rho_{M}=\rho_{M}(x) \mathrm{d} x_{0} \ldots \mathrm{d} x_{3}, \quad \rho_{M}(x)=\frac{4 R}{L_{N C}^{4} x_{4}} .
$$

Since the symplectic volume form is invariant, we have

$$
\int_{\mathcal{M}} \rho_{M} f(\nabla g)=-\int_{\mathcal{M}} \rho_{M}(\nabla f) g,
$$

which in Cartesian coordinates reduces to

$$
\int_{\mathcal{M}} \rho_{M} f x^{4}\left(\partial_{\mu} g\right)=-\int_{\mathcal{M}} \rho_{M} x^{4}\left(\partial_{\mu} f\right) g
$$

in accordance with (2.51).

Averaging over $\boldsymbol{S}^{\mathbf{2}}$. The above integral (2.49) over the entire bundle space $\mathbb{C} P^{1,2}$ can be viewed as an integral over the fiber followed by an integral over the base manifold $H^{4}$. Let us discuss the former in some detail: We define the averaging $[f(t)]_{0}$ as an integral over the $S^{2}$ fiber described by the $t$ generators,

$$
[f(t)]_{0}=\frac{1}{4 \pi r^{-2} \cosh (\eta)^{2}} \int_{S_{t}^{2}} f(t)
$$

such that $[1]_{0}=1$. Explicitly, the $\mathrm{SO}(4,1)$ covariant formula of $[40]$

$$
\left[\theta^{a b} \theta^{c d}\right]_{0}=\frac{r^{2} R^{2}}{3}\left(P^{a c} P^{b d}-P^{b c} P^{a d}+\varepsilon^{a b c d e} \frac{1}{R} x^{e}\right)
$$

with $P^{a c}=\eta^{a b}+R^{-2} x^{a} x^{b}$ gives, for example,

$$
\begin{aligned}
{\left[t^{\mu} t^{\nu}\right]_{0} } & =: \frac{1}{3 r^{2}} \kappa^{\mu \nu}, \\
\kappa^{\mu \nu} & =\cosh ^{2}(\eta) \eta^{\mu \nu}+\frac{x^{\mu} x^{\nu}}{R^{2}}, \quad \kappa_{\mu \nu} x^{\nu}=0 .
\end{aligned}
$$

Here $\kappa^{\mu \nu}$ is the unique $\mathrm{SO}(3,1)$-compatible, positive semi-definite metric which projects out the time-like directions. Similarly, one finds

$$
\begin{aligned}
{\left[t^{\alpha} \theta^{\mu \nu}\right]_{0} } & =-\frac{1}{3}\left(\sinh (\eta)\left(\eta^{\alpha \nu} x^{\mu}-\eta^{\alpha \mu} x^{\nu}\right)+x_{\beta} \varepsilon^{\beta 4 \alpha \mu \nu}\right) \\
{\left[t^{\mu_{1}} \ldots t^{\mu_{4}}\right]_{0} } & =\frac{3}{5}\left(\left[t^{\mu_{1}} t^{\mu_{2}}\right]\left[t^{\mu_{3}} t^{\mu_{4}}\right]_{0}+\left[t^{\mu_{1}} t^{\mu_{3}}\right]\left[t^{\mu_{2}} t^{\mu_{4}}\right]_{0}+\left[t^{\mu_{1}} t^{\mu_{4}}\right]\left[t^{\mu_{2}} t^{\mu_{3}}\right]_{0}\right) .
\end{aligned}
$$

More generally, one can derive a Wick-type formula

$$
\left[t^{\mu_{1}} \ldots t^{\mu_{n}}\right]_{0}=c_{n} \sum_{\text {contractions }}[t t]_{0} \ldots[t t]_{0} .
$$




\subsection{Metric properties of $\mathcal{M}^{3,1}$}

The effective metric on the background $Y^{\mu}=T^{\mu}(4.8)$ can be extracted from the kinetic term for the fluctuations, which in the matrix model has the universal form ${ }^{8}[1,36,44]$

$$
\begin{aligned}
S[\phi] & =R^{2} \operatorname{Tr}\left[T^{\mu}, \phi\right]\left[T_{\mu}, \phi\right] \sim-R^{2} \int_{\mathbb{C} P^{1,2}} \mathrm{~d} \Omega\left\{t^{\mu}, \phi\right\}\left\{t_{\mu}, \phi\right\} \\
& =-\int_{\mathcal{M}^{3,1}} \mathrm{~d} x_{0} \ldots \mathrm{d} x_{3} \rho_{M}(x) \gamma^{\mu \nu} \partial_{\mu} \phi \partial_{\nu} \phi \\
& =-\int_{\mathcal{M}^{3,1}} \mathrm{~d}^{4} x \sqrt{\left|G_{\mu \nu}\right|} G^{\mu \nu} \partial_{\mu} \varphi \partial_{\nu} \varphi
\end{aligned}
$$

using (2.49). Some dimensionful constants are absorbed in $\varphi$, and the last form is manifestly covariant..$^{9}$ Here

$$
\begin{aligned}
\gamma^{\alpha \beta} & =\eta_{\mu \nu} e^{\mu \alpha} e^{\nu \beta}=\sinh ^{2}(\eta) \eta^{\alpha \beta} \\
e^{\alpha} & =\left\{t^{\alpha}, \cdot\right\}=e^{\alpha \mu} \partial_{\mu}, \quad e^{\alpha \mu}=\eta^{\alpha \mu} \sinh (\eta),
\end{aligned}
$$

where $e^{\alpha}$ plays the role of a (rescaled) vielbein. The effective metric on $\mathcal{M}^{3,1}$ is then

$$
G^{\mu \nu}=\alpha \gamma^{\mu \nu}, \quad \alpha=\sqrt{\frac{1}{\tilde{\rho}_{M}^{2}\left|\gamma^{\mu \nu}\right|}}=\sinh ^{-3}(\eta)
$$

which is $\mathrm{SO}(3,1)$-invariant with signature $(-+++)$. Some irrelevant dimensionful constants have been dropped in $\tilde{\rho}_{M}$. Consequently, the inverse metric of $(2.62)$ is given by

$$
G_{\mu \nu}=\sinh (\eta) \eta_{\mu \nu}
$$

The metric is conformal to the induced (or closed-string) metric $\eta_{\mu \nu}$, hence the causal structures are the same.

FLRW form of the metric. Recall the coordinate choice on the 3-hyperboloid of [39],

$$
\left(\begin{array}{l}
x^{0} \\
x^{1} \\
x^{2} \\
x^{3}
\end{array}\right)=R \cosh (\eta)\left(\begin{array}{c}
\cosh (\chi) \\
\sinh (\chi) \sin (\theta) \cos (\varphi) \\
\sinh (\chi) \sin (\theta) \sin (\varphi) \\
\sinh (\chi) \cos (\theta)
\end{array}\right)
$$

Then the $\mathrm{SO}(1,3)$-invariant metric on $H_{\eta}^{3}$ has the form

$$
\left.\mathrm{d} s^{2}\right|_{H^{3}}=\sum_{i} \mathrm{~d} x_{i}^{2}=R^{2} \cosh ^{2}(\eta)^{2} \mathrm{~d} \Sigma^{2},
$$

\footnotetext{
${ }^{8}$ The effective metric on fuzzy spaces is an interesting topic that has been discussed from various points of view $[25,56,57]$. However on brane solutions in the matrix model, there is no choice but to use the present effective metric, which is encoded in the kinetic term of the action. This is consistent with the open string metric on D-branes [22, 59].

${ }^{9}$ The signs are chosen according to the standard form $\mathcal{L}=T-V$.
} 
where $\mathrm{d} \Sigma^{2}$ is the length element on a spatial standard 3-hyperboloid $H^{3}$. Therefore

$$
\begin{aligned}
\left.\mathrm{d} s_{G}^{2}\right|_{p}=G_{\mu \nu} \mathrm{d} x^{\mu} \mathrm{d} x^{\nu} & =-R^{2} \sinh ^{3}(\eta) \mathrm{d} \eta^{2}+R^{2} \sinh (\eta) \cosh ^{2}(\eta) \mathrm{d} \Sigma^{2} \\
& =-\mathrm{d} t^{2}+a^{2}(t) \mathrm{d} \Sigma^{2}
\end{aligned}
$$

where the scale parameter $a(t)$ is determined by the following two equations:

$$
\begin{aligned}
a(t)^{2} & =R^{2} \sinh (\eta) \cosh ^{2}(\eta), \\
\mathrm{d} t & =R \sinh (\eta)^{\frac{3}{2}} \mathrm{~d} \eta .
\end{aligned}
$$

The first equation gives

$$
2 a \mathrm{~d} a=R^{2} \cosh (\eta)\left(1+3 \sinh ^{2}(\eta)\right) \mathrm{d} \eta
$$

and combining these we obtain

$$
2 \frac{\mathrm{d} a}{\mathrm{~d} t}=\sinh ^{-2}(\eta)\left(1+3 \sinh ^{2}(\eta)\right)=3+\frac{1}{\sinh ^{2}(\eta)},
$$

which for late times gives

$$
a(t) \approx \frac{3}{2} t, \quad t \rightarrow \infty
$$

This describes a linear coasting universe as in [39, 44], cf. [60-62]. For early times, we can approximate

$$
R \eta^{\frac{3}{2}} d \eta=d t, \quad \eta \propto\left(t-t_{0}\right)^{\frac{2}{5}}
$$

such that

$$
a(t) \propto \eta^{\frac{1}{2}} \propto\left(t-t_{0}\right)^{\frac{1}{5}} .
$$

Hence we obtain a reasonable FLRW cosmology that is asymptotically coasting at late times and a Big Bang-like initial singularity, or rather a Big Bounce, since the flow of time is expected to be the opposite ${ }^{10}$ on the two sheets of $\mathcal{M}^{3,1}$.

We note that at late times, one can relate conformal factor $\alpha$ of (2.62) to the cosmic scale parameter $a(t)$ as follows

$$
\alpha \sim \sinh ^{-3}(\eta) \sim \frac{R^{3}}{x_{0}^{3}} \sim \frac{R^{2}}{a(t)^{2}} .
$$

Effective d'Alembertian. It follows from action (2.60) of a scalar field that the Laplacian (or rather d'Alembertian) on $\mathcal{M}^{3,1}$ associated to the metric $G_{\mu \nu}$ reads

$$
\begin{aligned}
\square & =-\left\{t^{\alpha},\left\{t_{\alpha}, \cdot\right\}\right\}=\alpha^{-1} \square_{G}, \\
\text { with } \quad \square_{G} & =-\frac{1}{\sqrt{\left|G_{\mu \nu}\right|}} \partial_{\mu}\left(\sqrt{\left|G_{\mu \nu}\right|} G^{\mu \nu} \partial_{\nu}\right) \\
& =-\gamma^{\alpha \beta} \partial_{\alpha} \partial_{\beta}-\sinh (\eta) \eta^{\alpha \beta}\left(\partial_{\alpha} \sinh (\eta)\right) \partial_{\beta} .
\end{aligned}
$$

\footnotetext{
${ }^{10}$ upon imposing a suitable i $\varepsilon$ prescription; work in progress.
} 
For example, we obtain

$$
\square_{G} f(\eta)=-\sinh (\eta) \partial^{\mu}\left(\sinh (\eta) \partial_{\mu}\right) f(\eta)=\frac{1}{R^{2}}\left(\partial_{\eta}^{2}+3 \frac{\sinh (\eta)}{\cosh (\eta)} \partial_{\eta}\right) f(\eta),
$$

which in particular results in

$$
\square\left(x^{\mu} x_{\mu}\right)=-2-10 \sinh ^{2}(\eta) \quad \text { and } \quad \square\left(x_{\mu}\right)=\frac{1}{R^{2}} x_{\mu} .
$$

Geometric structures and Lorentz invariance. Let us recapitulate the basic geometric structures on the semi-classical space-time $\mathcal{M}^{3,1}$ :

(1) The metric $\gamma^{\mu \nu}$ or its conformal class, with Minkowski signature, and the corresponding matrix or Poisson d'Alembertian $\square$ of (2.74). This allows to identify uniquely an effective metric $G^{\mu \nu}$ (2.62), which defines the meaning of local Lorentz invariance.

(2) The space-like metric $\kappa^{\mu \nu}(2.57)$ on the space-like sheets $H^{3}$.

(3) The time-like vector field

$$
\tau=x^{\mu} \partial_{\mu}=-x^{4} \frac{\partial}{\partial x^{4}},
$$

which is the comoving time derivative of the cosmic background, cf. (3.41).

All of these respect the $\mathrm{SO}(3,1)$ isometry. Notice that the anti-symmetric tensor $\theta^{\mu \nu}$ is not in this list, since it vanishes upon averaging $[\cdot]_{0}$ over the local fiber and, therefore, is not directly observable. This is a crucial advantage over noncommutative field theory on more basic spaces such as $\mathbb{R}_{\theta}^{4}[63,64]$. Clearly $\kappa_{\mu \nu}$ and $\tau$ separate the effective metric $G^{\mu \nu}$ into space-like and time-like components. This breaks local Lorentz invariance, but in a mild way, respecting the global $\mathrm{SO}(3,1)$ symmetry defined by the cosmic background. A crucial question is whether or not these Lorentz-breaking structures enter significantly into the local physics. We will see that at least the propagation of all physical higher-spin modes is indeed governed solely by $G^{\mu \nu}$.

\section{Wavefunctions, higher-spin modes and covariance}

Now consider the full endomorphism algebra $\mathcal{C}:=\operatorname{End}\left(\mathcal{H}_{n}\right)$, which is interpreted as quantized algebra of functions on $\mathbb{C} P^{1,2}$. This is in one-to-one correspondence with classical functions on $\mathbb{C} P^{1,2}$ via the quantization map (2.30). Due to the underlying bundle structure, $\mathcal{C}$ can be viewed as space of higher-spin harmonics over $\mathcal{M}^{3,1}$. In this section, we show how $\mathcal{C}$ separates into sub-sectors ${ }^{11} \mathcal{C}^{s}$, which correspond to spin $s$ harmonics. We will use the same symbols $\mathcal{C}, \mathcal{C}^{s}$ for the semi-classical and the fuzzy case.

We can define an $\mathrm{SO}(4,2)$-invariant inner product on $\mathcal{C}$ via

$$
\langle\phi, \psi\rangle=\operatorname{Tr}\left(\phi^{\dagger} \psi\right) \sim \int_{\mathbb{C} P^{1,2}} \phi^{\dagger} \psi, \quad \phi, \psi \in \operatorname{HS}\left(\mathcal{H}_{n}\right) .
$$

\footnotetext{
${ }^{11}$ In the semi-classical limit, the $\mathcal{C}^{s}$ are modules over $\mathcal{C}^{0}$. In the fuzzy case the $\mathcal{C}^{s}$ are no longer modules and hence do not fit into the standard scheme of noncommutative geometry, but nevertheless the interpretation is correct.
} 
For polynomial functions, this trace diverges; therefore, we will mostly consider the analogs of square-integrable functions i.e. the space of Hilbert-Schmidt operators $\operatorname{HS}\left(\mathcal{H}_{n}\right)$ in $\operatorname{End}\left(\mathcal{H}_{n}\right)$. The space $\operatorname{HS}\left(\mathcal{H}_{n}\right)$ is itself a Hilbert space and a Banach algebra; hence, the inner product is positive definite by construction. We will nevertheless use the somewhat sloppy notation $\phi \in \mathcal{C}$ while implicitly using $\phi \in \operatorname{HS}\left(\mathcal{H}_{n}\right)$.

\subsection{Spin Casimir}

We can now decompose the modes in $\mathcal{C}$ into unitary representations of $\mathrm{SO}(3,1) \subset$ $\mathrm{SO}(4,1) \subset \mathrm{SO}(4,2)$, organized as higher-spin modes. To achieve that, recall that the following spin Casimir on $H_{n}^{4}$ was introduced in [40]:

$$
\begin{aligned}
\mathcal{S}^{2} & :=\frac{1}{2} \sum_{a, b \neq 5}\left[\mathcal{M}_{a b},\left[\mathcal{M}^{a b}, \cdot\right]\right]+r^{-2}\left[X_{a},\left[X^{a}, \cdot\right]\right] \\
& =2 C^{2}[\mathfrak{s o}(4,1)]-C^{2}[\mathfrak{s o}(4,2)]
\end{aligned}
$$

which commutes with $\square_{H}$,

$$
\left[\mathcal{S}^{2}, \square_{H}\right]=0 \quad \text { with } \quad \square_{H}=\left[X_{a},\left[X^{a}, \cdot\right]\right]=r^{2}\left(C^{2}[\mathfrak{s o}(4,2)]-C^{2}[\mathfrak{s o}(4,1)]\right) .
$$

This means that $\square_{H}$ and $\mathcal{S}^{2}$ can be simultaneously diagonalized, defining the spin $s$ modes $\mathcal{C}^{s}$

$$
\operatorname{End}\left(\mathcal{H}_{n}\right)=\mathcal{C}=\mathcal{C}^{0} \oplus \mathcal{C}^{1} \oplus \ldots \oplus \mathcal{C}^{n} \quad \text { with }\left.\quad \mathcal{S}^{2}\right|_{\mathcal{C}^{s}}=2 s(s+1)
$$

as shown in [40]. Hence $\square_{H}$ defines a Laplacian on the $\mathcal{C}^{s}$, which are modules over the algebra $\mathcal{C}^{0}$ of functions on $H^{4}$. Moreover, the connection $\nabla$ of $(2.45)$ also respects $\mathcal{C}^{s}$. On the other hand, we observe that $\mathcal{S}^{2}$ also commutes with $\square$ which characterizes $\mathcal{M}_{n}^{3,1}$ :

$$
\left[\mathcal{S}^{2}, \square\right]=0 \quad \text { with } \quad \square=\square_{T} \equiv\left[T_{\mu},\left[T^{\mu}, \cdot\right]\right]=R^{-2}\left(C^{2}[\mathfrak{s o}(4,1)]-C^{2}[\mathfrak{s o}(3,1)]\right)
$$

because $\mathcal{S}^{2}$ respects $\mathrm{SO}(4,1)$. As a consequence, the decomposition (3.4) also provides the decomposition of functions on $\mathcal{M}_{n}^{3,1}$ into higher-spin modes, truncated at $n$. Although the space of functions is the same for $H_{n}^{4}$ and $\mathcal{M}_{n}^{3,1}$, the different geometry is encoded in the Laplacian or d'Alembertian. Here $\square_{T}$ defines a d'Alembertian on $\mathcal{M}_{n}^{3,1}$ and encodes an effective metric with Minkowski signature, while $\square_{H}$ defines a Laplacian on $H_{n}^{4}$ with Euclidean signature, as discussed in [40]. Both respect the same decomposition into higher spin modes $\mathcal{C}^{s}$.

Mode decomposition. Next, we can decompose $\mathcal{C}=\operatorname{End}\left(\mathcal{H}_{n}\right)$ into $\mathrm{SO}(4,2)$ irreps, which provides a decomposition into a direct sum or integral

$$
\mathcal{C}=\int \mathrm{d} \Lambda V_{\Lambda}^{\mathfrak{s o}(4,2)} \cong \mathcal{C}\left(\mathbb{C} P^{1,2}\right)
$$

of unitary irreps $V_{\Lambda}^{\mathfrak{s}(4,2)}$ of $\mathfrak{s o}(4,2)$. Due to $(2.30)$, this decomposition is multiplicity-free and uniquely identified by their quadratic Casimir $C^{2}(\mathfrak{s o}(4,2))$, as in the compact case. 
The $V_{\Lambda}^{\mathfrak{s o}(4,2)}$ can be further decomposed into a sum of $\mathfrak{s o}(4,1)$ irreps as follows:

$$
V_{\Lambda}^{\mathfrak{s o}(4,2)}=\bigoplus_{0 \leq s \leq n} V_{\Lambda, s}^{\mathfrak{s o}(4,1)}
$$

where the spin $s$ eigenvalue of (3.2) is restricted to integer values $0 \leq s \leq n$, as shown in [40]. The elements of $V_{\Lambda, s}^{\mathfrak{s o}(4,1)}$ can be viewed as spin $s$ fields ${ }^{12}$ on $H^{4}$ because $\mathbb{C} P^{1,2}$ is an equivariant $S^{2}$-bundle over $H^{4}$, and the harmonics on $S^{2}$ are captured by $s$. Finally, these $V_{\Lambda, s}^{\mathfrak{s o}(4,1)}$ can be further decomposed into $\mathfrak{s o}(3,1)$ irreps as

$$
\begin{aligned}
V_{\Lambda, s}^{\mathfrak{s o}(4,1)} & =\bigoplus_{m^{2}} V_{\Lambda, s, m^{2}}^{\mathfrak{s o}(3,1)} \\
\left(\square+m^{2}\right) \phi_{\Lambda, m^{2}}^{(s)} & =0, \quad m^{2}=R^{-2}\left(C^{2}[\mathfrak{s o}(4,1)]-C^{2}[\mathfrak{s o}(3,1)]\right)
\end{aligned}
$$

for some spectrum of eigenvalues $m^{2}$ which could a priori have any sign. Via the quantization map $\mathcal{Q}$ of (2.30), these correspond precisely to fields with mass $m^{2}$ on $\mathcal{M}^{3,1}$ with effective metric $G^{\mu \nu}$. Quite remarkably, we will see in section 5.4 that the on-shell, propagating modes in the present theory are precisely given in terms of eigenmodes of $\square$ with $m^{2}=0$. However they are expected to be non-trivial combinations of different $\Lambda$ modes.

Tensor generators and tensor fields. Now consider the fluctuation modes on $\mathcal{M}^{3,1}$ more explicitly. They can be expanded in terms of the $\theta^{a b}$ generators as follows:

$$
\begin{aligned}
\Phi & =\phi(X)+\phi_{a b}(X) \mathcal{M}^{a b}+\ldots \quad \in \operatorname{End}\left(\mathcal{H}_{n}\right) \\
& \sim \phi(x)+\phi_{a b}(x) m^{a b}+\ldots
\end{aligned}
$$

This will be refined below in terms of the $\theta^{\mu \nu}$ and/or $t^{\mu}$ generators. Then the coefficient functions $\phi_{a b}(x)$ etc. can be interpreted as functions (or higher-spin tensor fields) on ${ }^{13}$ $\mathcal{M}^{3,1}$, or more properly as sections of higher-spin bundles on $H^{4}$. Again, this expansion is truncated at $n$.

Due to the constraints of $\mathcal{M}^{a b}$, these coefficient functions are not independent, and there are various possibilities how to parametrize the most general modes. For $H^{4}$, this has been achieved in [40]. To make this more transparent, we will focus on the semi-classical limit from now on. Then $\mathcal{C}^{0}$ reduces to the space of functions on $H^{4}$ resp. $\mathcal{M}^{3,1}$, and the $\mathcal{C}^{s}$ are modules over $\mathcal{C}^{0}$. The Casimir $\mathcal{S}^{2}$ then measures the spin of the local $\mathrm{SU}(2)_{L}$ acting along the fiber.

\subsection{Space-like gauge and $\mathrm{SO}(3,1)$-covariant tensor fields}

Now we establish a correspondence of the $\mathcal{C}^{s}$ with the standard representation of higher-spin fields on $\mathcal{M}^{3,1}$. We can define a map

$$
\begin{aligned}
\Gamma^{(s)} \mathcal{M}^{3,1} & \rightarrow \mathcal{C}^{s} \\
\phi_{\mu_{1} \ldots \mu_{s}}^{(s)}(x) & \mapsto \phi_{\mu_{1} \ldots \mu_{s}}^{(s)}(x) t^{\mu_{1}} \ldots t^{\mu_{s}}
\end{aligned}
$$

\footnotetext{
${ }^{12}$ Note that $\mathrm{SO}(4,2)$ should not be interpreted as conformal group on $H^{4}$, rather it provides the organization of the higher-spin modes on $H^{4}$ and $\mathcal{M}^{3,1}$.

${ }^{13}$ We will ignore the dependence on two sheets $\mathcal{M}^{ \pm}$for simplicity.
} 
from rank $s$ traceless symmetric tensor field $\phi_{\mu_{1} \ldots \mu_{s}}^{(s)}$ on $\mathcal{M}^{3,1}$ to $\mathcal{C}^{s}$. Due to the constraint $x_{\mu} t^{\mu}=0$ of (2.36) the map (3.10) has a kernel, which can be viewed as an internal gauge invariance

$$
\phi_{\mu_{1} \ldots \mu_{s}} \rightarrow \phi_{\mu_{1} \ldots \mu_{s}}+x_{\mu_{1}} \phi_{\mu_{2} \ldots \mu_{s}} .
$$

We can use this to impose the following gauge-fixing condition

$$
x^{\mu_{i}} \phi_{\mu_{1} \ldots \mu_{s}}=0
$$

denoted as space-like gauge. This gauge-fixing respects $\mathrm{SO}(3,1)$, but is not covariant in the standard sense because it uses the time-like vector field $\tau=x^{\mu} \partial_{\mu}$ of (2.78). Hence, (3.12) is reminiscent of the Coulomb or radiation gauge. In particular, the gauge-fixing implies that all time-components of $\phi_{\mu_{1} \ldots \mu_{s}}$ vanish, such that it defines a tensor field on the timeslices $H^{3}$. Assuming this space-like gauge, we can write down an inverse map to (3.10) by

$$
\begin{aligned}
\mathcal{C}^{s} & \rightarrow \Gamma^{(s)} \mathcal{M}^{3,1} \\
\phi^{(s)} & \rightarrow \phi_{\mu_{1} \ldots \mu_{s}}^{(s)}(x)
\end{aligned}
$$

resulting in a traceless symmetric space-like tensor field. Note that (3.13) is a $\mathcal{C}^{0}$-module map, unlike the analogous map given in [40] for $H^{4}$. To see that the map (3.13) is surjective, it suffices to express the $\mathcal{M}^{\mu \nu}$ in terms of $t^{\mu}$ generators using (2.43). This establishes a one-to-one parametrization of $\mathcal{C}^{s}$ in terms of traceless symmetric space-like tensor fields $\phi_{\mu_{1} \ldots \mu_{s}}$, which is very useful to count degrees of freedom. More generally, one can also define maps

$$
\begin{aligned}
\mathcal{C}^{s} & \rightarrow \Gamma^{(1)} \mathcal{M}^{3,1} \otimes \mathcal{C}^{s-1} \\
\phi^{(s)} & \rightarrow \phi_{\mu}^{(s)}:=\phi_{\mu_{1} \ldots \mu_{s}}^{(s)}(x) t^{\mu_{2}} \ldots t^{\mu_{s}}
\end{aligned}
$$

etc., but we will avoid such constructions because they do not respect the underlying higher symmetry structures.

Note that the internal gauge invariance (3.11) is consistent with the positive definite inner product (3.1). For example,

$$
\left\langle\phi_{\mu}(x) t^{\mu}, \psi_{\nu}(x) t^{\nu}\right\rangle=\frac{1}{3 r^{2}}\left\langle\phi_{\mu}^{\dagger}(x) \psi_{\nu}(x)\right\rangle \kappa^{\mu \nu}
$$

using (2.56) and the definition (2.57) of $\kappa^{\mu \nu}$. The expression (3.15) vanishes on the wouldbe pure gauge states $x_{\mu_{1}} \phi_{\mu_{2} \ldots \mu_{s}}$ in (3.11), and is manifestly positive for fields satisfying the gauge-fixing condition (3.12). Hence the Hilbert space structure inherited from $\mathcal{H}_{n}$ takes care of the above gauge freedom, and the would-be pure gauge states are already factored out. This is a remarkable aspect of the present framework.

\section{3 $\mathrm{SO}(3,1)$ intertwiners and sub-structure}

The above organization can be refined further by considering the $\mathrm{SO}(3,1)$ intertwiner

$$
\begin{aligned}
D:=-\mathrm{i}\left[X_{4}, \cdot\right] \sim\left\{x^{4}, \cdot\right\}: \quad & V_{\Lambda}^{\mathfrak{s o}(4,2)} \rightarrow V_{\Lambda}^{\mathfrak{s o}(4,2)} \\
& V_{\Lambda, s}^{\mathfrak{s o}(4,1)} \mapsto V_{\Lambda, s+1}^{\mathfrak{s o}(4,1)} \oplus V_{\Lambda, s-1}^{\mathfrak{s o}(4,1)}
\end{aligned}
$$


which is anti-Hermitian in the sense

$$
\int \phi^{\left(s^{\prime}\right)} D \phi^{(s)}=-\int D \phi^{\left(s^{\prime}\right)} \phi^{(s)} .
$$

Since $D$ is a derivation, it is determined by its action on the generators

$$
D\left(X^{\mu}\right)=r^{2} R T^{\mu} \quad \text { and } \quad D\left(T^{\mu}\right)=R^{-1} X^{\mu},
$$

which is essentially an exchange of $X^{\mu}$ and $T^{\mu}$. D is a non-compact generator of $\mathrm{SO}(4,2)$, which transforms as vector under $\mathrm{SO}(4,1)$. Hence in unitary representations, it should be unbounded in both directions. Note that $D$ does not commute with $\mathcal{S}^{2}$, and it changes ${ }^{14}$ the quantum number $s$ by \pm 1 , cf. (A.38). We can therefore separate $D$ into raising and lowering operators

$$
D=D^{+}+D^{-}: \quad \mathcal{C}^{s} \rightarrow \mathcal{C}^{s+1} \oplus \mathcal{C}^{s-1}
$$

where

$$
\begin{aligned}
D^{-}=-\mathrm{i}\left[X_{4}, \cdot\right]_{-}: & \mathcal{C}^{s} \rightarrow \mathcal{C}^{s-1}, & D^{-} \phi^{(s)}:=-\mathrm{i}\left[X_{4}, \phi^{(s)}\right]_{s-1} \\
D^{+}=-\mathrm{i}\left[X_{4}, \cdot\right]_{+}: & \mathcal{C}^{s} \rightarrow \mathcal{C}^{s+1}, & D^{+} \phi^{(s)}:=-\mathrm{i}\left[X_{4}, \phi^{(s)}\right]_{s+1}
\end{aligned}
$$

and similarly in the Poisson case. Since modes with different spin are orthogonal, it follows that

$$
D^{+}=-\left(D^{-}\right)^{\dagger}
$$

Hence $D^{+} D^{-}$respects the spin and can be diagonalized within $V_{\Lambda}^{\mathfrak{s o}(4,2)}$. Next, define the subspaces

$$
\mathcal{K}^{(s, k)}:=\left\{\phi^{(s)} \in \mathcal{C}^{s} \mid \quad\left(D^{-}\right)^{k+1} \phi^{(s)}=0\right\} \subset \mathcal{C}^{s}
$$

and the quotients

$$
\mathcal{C}^{(s, k)}:=\mathcal{K}^{(s, k)} / \mathcal{K}^{(s, k-1)}
$$

As we will see below, $D^{-}$is essentially the 3 -divergence on the space-like leaves $H^{3} \subset \mathcal{M}^{3,1}$. Thus according to $(3.13), \mathcal{C}^{(s, 0)}$ is the space of divergence-free space-like rank s symmetric tensor fields, and the intertwiners $D^{ \pm}$act as follows

$$
D^{ \pm}: \quad \mathcal{C}^{(s, k)} \rightarrow \mathcal{C}^{(s-1, k-1)} .
$$

Now the orthogonal Hilbert space decomposition

$$
\mathcal{H}=\operatorname{Ker}(A) \oplus \operatorname{Im}\left(A^{\dagger}\right)
$$

for $A=D^{-}$gives

$$
\mathcal{C}^{(s)}=\mathcal{C}^{(s, 0)} \oplus \operatorname{Im}\left(D^{+}\right) .
$$

\footnotetext{
${ }^{14}$ This can be seen easily by looking at polynomials.
} 
Repeating this argument for $A=\left(D^{-}\right)^{k}$, we arrive at the orthogonal decomposition

$$
\mathcal{C}^{(s)}=\mathcal{C}^{(s, 0)} \oplus \mathcal{C}^{(s, 1)} \oplus \ldots \oplus \mathcal{C}^{(s, s)}
$$

where $D^{-}$resp. $D^{+}$act as isomorphism

$$
D^{-}: \mathcal{C}^{(s, k)} \rightarrow \mathcal{C}^{(s-1, k-1)}, \quad D^{+}: \mathcal{C}^{(s, k)} \rightarrow \mathcal{C}^{(s+1, k+1)} .
$$

Therefore

$$
\mathcal{C}^{(s, k)} \cong \mathcal{C}^{(s-k, 0)}
$$

correspond to symmetric traceless space-like divergence-free spin $(s-k)$ tensor fields.

Let us emphasize that there are two different notions of spin in the current context: on the one hand, the quantum number $s$ labels the Casimir $\mathcal{S}^{2}$ eigenvalue, which measures the spin on the internal $S^{2}$ fiber. On the other hand, as tensor fields on $H^{3}$, the $\operatorname{SO}(3,1)$ group theory yields another notion of spin, and in this sense the $\mathcal{C}^{(s, k)}$ are spin $(s-k)$ irreps of $\mathrm{SO}(3,1)$.

Furthermore, the inner products between the quotients (3.23) have the following structure:

$$
\left\langle\mathcal{C}^{(s, k)}, \mathcal{C}^{\left(s^{\prime}, k^{\prime}\right)}\right\rangle=c_{(s, k)} \delta_{k^{\prime}}^{k} \delta_{s^{\prime}}^{s}, \quad c_{(s, k)}>0
$$

In view of this structure, it may be tempting to consider the $\mathcal{C}^{(s-k, 0)}$ as analogs of primary fields, and the $\mathcal{C}^{(s, k)}$ for $k>0$ as descendants. However, this notion would be quite different from the familiar notion in CFT, where primaries are annihilated by special conformal transformations and are lowest weight modes for $D=-\mathrm{i}\left[X^{4}, \cdot\right]$. Here, $D$ is unbounded in both directions due to the rigid $\mathrm{SO}(4,2)$ signature, and $D^{-}$is the lowering operator. We will therefore refrain from using such a language, and note that the organization is quite different from CFT even though $\mathrm{SO}(4,2)$ plays a central role.

Finally, we observe that $D$ provides the missing part of the $\mathrm{SO}(4,1)$-invariant Laplace operator on $H^{4}$

$$
\square_{H}=\sum_{a=0}^{4}\left[X^{a},\left[X_{a}, \cdot\right]\right]=\left[X_{\mu},\left[X^{\mu}, \cdot\right]\right]-D^{2} .
$$

$\boldsymbol{D}^{ \pm}$and divergence. Now consider $D$ more explicitly. Due to (2.34), we have

$$
\begin{aligned}
2 x_{4} D(\phi)=\left\{x_{4}^{2}, \phi\right\} & =-\left\{x_{\mu} x^{\mu}, \phi\right\}=-2 x_{\mu}\left\{x^{\mu}, \phi\right\} \\
& =r^{2} R^{2}\left\{t^{\mu} t_{\mu}, \phi\right\}=2 r^{2} R^{2} t^{\mu}\left\{t_{\mu}, \phi\right\} .
\end{aligned}
$$

Assuming space-like gauge, $D$ takes the explicit form

$$
\begin{aligned}
D(\phi) & =\frac{r^{2} R^{2}}{x_{4}} t^{\mu}\left\{t_{\mu}, \phi\right\}=\frac{r^{2} R^{2}}{x_{4}} t^{\mu}\left(\sinh (\eta) \partial_{\mu} \phi_{\mu_{1} \ldots \mu_{s}}(x) t^{\mu_{1}} \ldots t^{\mu_{s}}-\frac{s}{r^{2} R^{2}} \phi_{\mu_{1} \ldots \mu_{s}} \theta^{\mu \mu_{1}} t^{\mu_{2}} \ldots t^{\mu_{s}}\right) \\
& =r^{2} R t^{\mu} \partial_{\mu} \phi_{\mu_{1} \ldots \mu_{s}} t^{\mu_{1}} \ldots t^{\mu_{s}} \equiv r^{2} R\left(\nabla_{\mu}^{(3)} \phi_{\mu_{1} \ldots \mu_{s}}\right) t^{\mu} t^{\mu_{1}} \ldots t^{\mu_{s}}
\end{aligned}
$$


using (2.36), where $\nabla^{(3)}$ is the covariant derivative along the $H^{3}$ sheets. The last form holds because the $t^{\mu}$ generators are space-like. Then (3.33) splits according to (3.20) into

$$
\begin{aligned}
D=D^{-}+D^{+}: \quad \mathcal{C}^{s} & \rightarrow \mathcal{C}^{s-1} \oplus \quad \mathcal{C}^{s+1} \\
\phi & \mapsto r^{2} R\left[t^{\mu} \nabla_{\mu}^{(3)} \phi\right]_{-}+r^{2} R\left[t^{\mu} \nabla_{\mu}^{(3)} \phi\right]_{+},
\end{aligned}
$$

where $D^{+}$is the symmetrized total derivative along $H^{3}$, and $D^{-}$is the 3 -divergence,

$$
D^{-} \phi^{(s)}=\beta_{s} r^{2} R \nabla_{(3)}^{\mu} \phi_{\mu \mu_{2} \ldots \mu_{s}} t^{\mu_{2}} \ldots t^{\mu_{s}}=: \beta_{s} r^{2} R \operatorname{div}_{3}\left(\phi^{(s)}\right) \quad \in \mathcal{C}^{s-1}
$$

for some $\beta_{s}$, and $\phi_{\mu \mu_{1} \ldots \mu_{s}}$ is totally symmetric and space-like. Then (3.35) is the space-like divergence of tensor fields on $H^{3} \subset H^{4}$; since the tensor fields are viewed as tensor fields on $H^{4}$ in space-like gauge, this can also be viewed as 4-dimensional divergence on $H^{4}$. Then $D^{+}$is determined via (3.21).

Usually, derivative modes $\mathcal{C}^{(s, k)}$ with $k>0$ would be considered as pure gauge fields. However here, all these modes are space-like and participate in the positive definite inner product (3.30). Such modes will be part of the physical Hilbert space. On the other hand, the divergence-free fields $D^{-} \phi^{(s, 0)}=0$ will constitute the massless spin $s$ fields from the 4 -dimensional point of view. For example, the $\phi^{(2,0)}$ modes correspond to divergence-free rank 2 traceless symmetric tensor fields $\phi_{\mu \nu}$ in space-like gauge, which clearly have only 2 independent degrees of freedom, as appropriate for massless gravitons. This somewhat unusual organization reflects the absence of manifest (local) Lorentz invariance.

In the following sections we will encounter the operator $D^{-} D^{+}$, which is essentially the 3-dimensional Laplacian on $H^{3}$,

$$
D^{-} D^{+} \phi^{(s)} \propto\left(\Delta_{(3)}+c_{s}\right) \phi^{(s)}
$$

where $\Delta_{(3)}=\nabla_{(3)}^{\alpha} \nabla_{(3) \alpha}$. For $s=0$, this is explicitly

$$
\begin{aligned}
D^{-} D^{+} \phi^{(0)} & =r^{2} R D^{-}\left(t^{\alpha} \partial_{\alpha} \phi^{(0)}\right)=r^{4} R^{2}\left[t^{\alpha} t^{\beta} \nabla_{\alpha}^{(3)} \partial_{\beta} \phi^{(0)}\right]_{0} \\
& =\frac{r^{2} R^{2}}{3} \kappa^{\alpha \beta} \nabla_{\alpha}^{(3)} \partial_{\beta} \phi^{(0)}=\frac{r^{2} R^{2}}{3} \cosh ^{2}(\eta) \Delta^{(3)} \phi^{(0)}
\end{aligned}
$$

using (2.56). Similarly for $s=1$, the 3 -divergence is

$$
\begin{aligned}
D^{-} \phi^{(1)} & =r^{2} R\left[t^{\alpha} \nabla_{\alpha}^{(3)} \phi^{(1)}\right]_{0}=r^{2} R\left[t^{\mu} t^{\alpha}\right]_{0} \nabla_{\alpha}^{(3)} \phi_{\mu} \\
& =\frac{R}{3} \cosh ^{2}(\eta) \nabla_{(3)}^{\mu} \phi_{\mu}
\end{aligned}
$$


which gives $\beta_{1}=\frac{1}{3 r^{2}} \cosh ^{2}(\eta)$ in (3.35). If $\phi^{(1)}$ is divergence-free, then

$$
\begin{aligned}
D^{-} D^{+} \phi^{(1)} & =r^{2} R D^{-}\left[t^{\alpha} \nabla_{\alpha}^{(3)} \phi^{(1)}\right]_{2} \\
& =r^{2} R D^{-}\left(\nabla_{\alpha}^{(3)} \phi_{\mu}\left[t^{\mu} t^{\alpha}\right]_{2}\right) \\
& =r^{4} R^{2}\left[t^{\beta} \nabla_{\beta}^{(3)} \nabla_{\alpha}^{(3)} \phi_{\mu}\left(t^{\mu} t^{\alpha}-\frac{1}{3 r^{2}} \kappa^{\mu \alpha}\right)\right]_{1} \\
& =\frac{4}{3} r^{2} R^{2} \cosh ^{2}(\eta)\left(\nabla_{(3)}^{\alpha} \nabla_{\alpha}^{(3)} \phi_{\mu} t^{\mu}+\nabla_{\mu(3)} \nabla_{\alpha}^{(3)} \phi^{\mu} t^{\alpha}\right) \\
& =\frac{4}{3} r^{2} R^{2} \cosh ^{2}(\eta)\left(\Delta^{(3)} \phi_{\mu} t^{\mu}+\left(R_{\mu \alpha}^{(3)}\right)_{\nu}^{\mu} \phi^{\nu} t^{\alpha}\right) \\
& =\frac{4}{3} r^{2}\left(R^{2} \cosh ^{2}(\eta) \Delta^{(3)} \phi^{(1)}+2 \phi^{(1)}\right)
\end{aligned}
$$

noting that $\nabla^{(3)} \kappa_{\mu \nu}=0$ and $\left[\nabla_{\mu}^{(3)}, \nabla_{\beta}^{(3)}\right] \phi^{\mu}=\left(R_{\mu \beta}^{(3)}\right)_{\nu}^{\mu} \phi^{\nu}=R_{\beta \nu}^{(3)} \phi^{\nu}$, where $R_{\beta \mu}^{(3)}=$ $\frac{2}{R^{2} \cosh ^{2}(\eta)} P_{\beta \mu}^{(3)}$ is the Ricci tensor on $H^{3}$. Here $P^{(3)}$ is the tangential projector on $H^{3}$.

Time-like vector field $\tau$ as intertwiner. Next, we consider

$$
\begin{aligned}
t_{\mu}\left\{x^{\mu}, \phi^{(s)}\right\} & =\theta^{\mu \nu} \partial_{\nu} \phi_{\mu_{1} \ldots \mu_{s}}(x) t^{\mu} t^{\mu_{1}} \ldots t^{\mu_{s}}-s \sinh (\eta) \phi_{\mu \mu_{2} \ldots \mu_{s}}(x) t^{\mu} t^{\mu_{2}} \ldots t^{\mu_{s}} \\
& =-\sinh (\eta)\left(s+x^{\nu} \partial_{\nu}\right) \phi^{(s)} .
\end{aligned}
$$

Therefore the time-like vector field $\tau=x^{\nu} \partial_{\nu}$ of $(2.78)$ on $\mathcal{M}^{3,1}$ can be extended as an $\mathrm{SO}(3,1)$ intertwiner of $\mathcal{C}^{s}$ as follows

$$
t^{\mu}\left\{x_{\mu}, \phi^{(s)}\right\}=-\sinh (\eta)(s+\tau) \phi^{(s)}=-x^{\mu}\left\{t_{\mu}, \phi^{(s)}\right\} \quad \in \mathcal{C}^{s} .
$$

Space-like gauge and degrees of freedom. The present space-like gauge is also useful to identify the independent physical degrees of freedom contained in $\mathcal{C}^{s}$. Consider e.g. $s=1$. Then $\phi^{(1)}=\phi_{\mu} t^{\mu}$ is a symmetric traceless space-like tensor field $\phi_{\mu}$. This separates into $\phi^{(1)}=\phi^{(1,0)}+\phi^{(1,1)}$, where $\phi^{(1,1)}=D^{+} \phi^{(0)}$ encodes a scalar field, and $\phi^{(1,0)} \in \mathcal{C}^{(1,0)}$ is 3-divergence-free $\nabla_{(3)}^{\mu} \phi_{\mu}^{(1,0)}=0$. This implies $\nabla^{\mu} \phi_{\mu}^{(1,0)}=0$ for any 4-dimensional $\mathrm{SO}(3,1)$ invariant connection on $\mathcal{M}^{3,1}$, because $x^{\mu} \phi_{\mu}=0$. Therefore $\phi^{(1,0)}$ provides the 2 degrees of freedom of a massless spin 1 gauge field, while $\phi \in \mathcal{C}^{(1,1)}$ contributes the remaining degree of freedom for a massive spin 1 field with 3 degrees of freedom.

Similarly for $s=2$, the $\phi^{(2)}=\phi_{\mu \nu} t^{\mu} t^{\nu}$ are symmetric traceless space-like tensor fields. The $\phi^{(2,0)}$ are 3-divergence-free $\nabla_{(3)}^{\mu} \phi_{\mu \nu}^{(2,0)}=0$, which implies $\nabla^{\mu} \phi_{\mu \nu}^{(2,0)}=0$ for any 4-dimensional $\mathrm{SO}(3,1)$-invariant connection on $\mathcal{M}^{3,1}$. Thus the $\phi_{\mu \nu}^{(2,0)}$ are traceless, divergence-free and space-like, hence they contain 2 degrees of freedom, as appropriate for gravitons. The remaining sectors $\phi^{(2,1)}=D^{+} \phi^{\prime(1,0)}$ and $\phi^{(2,2)}=D^{+} D^{+} \phi^{\prime(0)}$ in $\mathcal{C}^{2}$ provide the missing degrees of freedom for a general massive spin 2 field with 5 degrees of freedom. We will see in section 6 how this applies to the actual gravitons in the matrix model.

Gauge transformations and diffeomorphisms. Gauge transformations act on fields $\phi \in \mathcal{C}$ via $\phi \mapsto\{\Lambda, \phi\}$, for any $\Lambda \in \mathcal{C}$. For $\Lambda \in \mathcal{C}^{0}$, these correspond to (noncommutative) 
$\mathrm{U}(1)$ gauge transformations. For $\Lambda \in \mathcal{C}^{1}$, they induce volume-preserving diffeomorphisms (and corresponding gauge modes) on $H_{n}^{4}$, as discussed in [40]. On $\mathcal{M}^{3,1}$, the gauge transformations generated by a vector field $v^{\mu}$ (in space-like gauge) act on functions $\phi \in \mathcal{C}^{0}$ as

$$
\begin{aligned}
\left\{v^{\mu} t_{\mu}, \phi\right\}_{0} & =\left[t^{\mu} \theta^{\alpha \beta}\right]_{0} \partial_{\alpha} v_{\mu} \partial_{\beta} \phi+v^{\mu}\left\{t_{\mu}, \phi\right\}_{0} \\
& =\frac{1}{3}\left(\sinh (\eta)\left(\eta^{\mu \alpha} x^{\beta}-\eta^{\mu \beta} x^{\alpha}\right)-x_{\gamma} \varepsilon^{\gamma 4 \mu \alpha \beta}\right) \partial_{\alpha} v_{\mu} \partial_{\beta} \phi+\sinh (\eta) v^{\mu} \partial_{\mu} \phi \\
& =\frac{1}{3}\left(\sinh (\eta)\left(3 v^{\mu} \partial_{\mu}+(\operatorname{div} v) \tau-\left(\tau v^{\mu}\right) \partial_{\mu}\right) \phi+x_{\gamma} \varepsilon^{\gamma \mu \alpha \beta} \partial_{\alpha} v_{\mu} \partial_{\beta} \phi\right)
\end{aligned}
$$

using (2.58). Although this is not the most general diffeomorphism, it does include time-like derivatives on $\mathcal{M}^{3,1}$ via $\operatorname{div} v$, even though $v_{\mu}$ is in space-like gauge. The non-standard form reflects the existence of an invariant volume form on $\mathbb{C} P^{1,2}$. Nevertheless, this provides a powerful constraint for the resulting gravity theory that will be exploited in section 6.3.

\section{4 $\mathrm{SO}(4,1)$-covariant gauge and tensor fields on $H^{4}$}

Instead of the above $\mathrm{SO}(3,1)$-covariant organization in terms of tensor fields in space-like gauge, one can alternatively use a $\mathrm{SO}(4,1)$-covariant realization of $\phi^{(s)} \in \mathcal{C}^{s}$ in terms of tangential traceless divergence-free tensor fields on $H^{4}$ as in [40]. They are defined by

$$
\phi_{a_{1} \ldots a_{s}}^{H} \propto\left\{x^{a_{1}}, \ldots\left\{x^{a_{s}}, \phi^{(s)}\right\} \ldots\right\}_{0}
$$

normalized such that

$$
\phi^{(s)}=\left\{x^{a_{1}}, \ldots\left\{x^{a_{s}}, \phi_{a_{1} \ldots a_{s}}^{H}\right\} \ldots\right\} .
$$

The normalization factor is found recursively from ${ }^{15}$

$$
-\left\{x_{a}, \phi^{(s)}\right\}_{s-1}=\alpha_{s}\left(\square_{H}-2 r^{2}\right) \phi_{a}^{H(s-1)}
$$

where $\alpha_{1}=\frac{1}{3}, \alpha_{2}=\frac{2}{5}$, and so on and so forth. The $\phi_{a_{1} \ldots a_{s}}^{H}$ contain the same information as the $\phi_{\mu_{1} \ldots \mu_{s}}$ in space-like gauge, but enjoy more powerful symmetries. In particular, it follows that

$$
\begin{aligned}
-\left\{x^{a},\left\{x_{a}, \phi^{(s)}\right\}_{-}\right\}_{+} & =\alpha_{s}\left\{x^{a},\left(\square_{H}-2 r^{2}\right) \phi_{a}^{H(s-1)}\right\} \\
& =\alpha_{s}\left(\square_{H}-2 r^{2}(s+1)\right)\left\{x^{a}, \phi_{a}^{H(s-1)}\right\} \\
& =\alpha_{s}\left(\square_{H}-2 r^{2}(s+1)\right) \phi^{(s)}, \\
-\left\{x^{a},\left\{x_{a}, \phi^{(s)}\right\}_{+}\right\}_{-} & =\left(\left(1-\alpha_{s}\right) \square_{H}+2(s+1) \alpha_{s}\right) \phi^{(s)}
\end{aligned}
$$

using the intertwiner properties (A.57). In particular, the operators on the right-hand-side of (3.46) and (3.47) are positive, because the left-hand-side is positive ${ }^{16}$ on $H^{4}$. Reduced to $\mathcal{M}^{3,1}$, this gives

$$
-\left\{x^{\mu},\left\{x_{\mu}, \phi^{(s)}\right\}_{-}\right\}_{+}=\left(\alpha_{s}\left(\square_{H}-2 r^{2}(s+1)\right)+D^{+} D^{-}\right) \phi^{(s)} .
$$

\footnotetext{
${ }^{15}$ The notation here is slightly different from [40], where $\phi_{a}^{H(s-1)}$ is denoted as $\phi_{a}^{(s)}$.

${ }^{16}$ since $\int_{H^{4}}\left\{x^{a}, \phi\right\}_{-}\left\{x_{a}, \phi\right\}_{-}>0$, because $\left\{x_{a},.\right\}$ is tangential on the Euclidean space $H^{4}$, cf. [40].
} 
Similarly, one finds

$$
\begin{aligned}
& \left\{x^{\mu},\left\{x_{\mu}, \phi^{(s)}\right\}_{-}\right\}_{-}=-\left(\left(\square_{H}+\left\{x^{4},\left\{x^{4}, .\right\}\right\}\right) \phi^{(s)}\right)_{s-2}=-D^{-} D^{-} \phi^{(s)} \\
& \left\{x^{\mu},\left\{x_{\mu}, \phi^{(s)}\right\}_{+}\right\}_{+}=-D^{+} D^{+} \phi^{(s)}
\end{aligned}
$$

since $\left\{x^{\mu}, \cdot\right\}: \mathcal{C}^{s} \rightarrow \mathcal{C}^{s-1} \oplus \mathcal{C}^{s+1}$ while $\square_{H}: \mathcal{C}^{s} \rightarrow \mathcal{C}^{s}$. Combining these, we obtain

$$
-\left\{x^{\mu},\left\{x_{\mu}, \phi^{(s)}\right\}_{+}\right\}_{-}=\left(\left(1-\alpha_{s}\right) \square_{H}+2 \alpha_{s} r^{2}(s+1)+D^{-} D^{+}\right) \phi^{(s)} .
$$

To summarize, $\phi^{(s)}$ corresponds to a 4-dimensional spin $s$ field, either represented as divergence-free symmetric traceless tensor on $H^{4}$ or as space-like symmetric trace-less tensor on $\mathcal{M}^{3,1}$ which is generically not divergence-free. The counting of degrees of freedom is the same in both interpretations, and gives, for example, 5 in the case of $s=2$.

\section{Matrix model and quantum space-time solutions}

Let us move towards the matrix model, by considering the bosonic part of the action of the IKKT matrix model supplemented with a mass term

$$
S[Y]=\frac{1}{g^{2}} \operatorname{Tr}\left(\left[Y^{\mu}, Y^{\nu}\right]\left[Y^{\mu^{\prime}}, Y^{\nu^{\prime}}\right] \eta_{\mu \mu^{\prime}} \eta_{\nu \nu^{\prime}}-\mu^{2} Y_{\mu} Y^{\mu}\right)
$$

Here $\eta_{\mu \nu}=\operatorname{diag}(-1,1,1,1)$ is the flat Minkowski metric of the target space $\mathbb{R}^{1,3}$, and $\mu^{2}$ introduces a mass scale. This action leads to the classical equations of motion

$$
\begin{aligned}
\square_{Y} Y^{\mu}+\frac{1}{2} \mu^{2} Y^{\mu} & =0 \\
\text { with } \quad \square_{Y} & =\left[Y^{\mu},\left[Y_{\mu}, \cdot\right]\right] \sim-\left\{y^{\mu},\left\{y_{\mu}, \cdot\right\}\right\},
\end{aligned}
$$

where $\square_{Y}$ plays the role of the Laplacian or rather d'Alembertian operator. We consider the following ansatz for solutions of (4.2)

$$
Y^{\mu}=\mathcal{M}^{\mu a} \alpha_{a}, \quad \mu=0,1,2,3
$$

where $\alpha_{a}$ is some constant $\mathrm{SO}(4,2)$ vector. These describe homogeneous and isotropic quantized cosmological space-times $\mathcal{M}_{n}^{1,3}$ as introduced ${ }^{17}$ in [39]. Since $\eta^{\mu \nu}$ is $\mathrm{SO}(3,1)$ invariant, we have

$$
\begin{aligned}
{\left[Y_{\rho},\left[Y^{\rho}, Y^{\mu}\right]\right] } & =\mathrm{i}(\alpha \cdot \alpha)\left[Y_{\rho}, \mathcal{M}^{\rho \mu}\right]=-\mathrm{i}(\alpha \cdot \alpha)\left[\mathcal{M}^{\rho \mu}, Y_{\rho}\right] \\
& =(\alpha \cdot \alpha)\left\{\begin{array}{l}
Y^{\mu}, \mu \neq \rho \\
0, \quad \mu=\rho
\end{array} \quad\right. \text { (no sum) }
\end{aligned}
$$

and therefore

$$
\square_{Y} Y^{\mu}=3(\alpha \cdot \alpha) Y^{\mu}
$$

\footnotetext{
${ }^{17}$ We change notation from [39], where $Y^{1}$ was dropped instead of $Y^{4}$.
} 
Consequently, we obtain three different types of quantized space-time solutions with Minkowski signature in the IKKT model with mass term,

$$
\begin{aligned}
& \square_{X} X^{\mu}=-3 r^{2} X^{\mu}, \quad \text { for } \quad \frac{1}{2} \mu^{2}=3 r^{2} \\
& \square_{T} T^{\mu}=3 R^{-2} T^{\mu}, \quad \text { for } \quad \frac{1}{2} \mu^{2}=-3 R^{-2} \\
& \square_{Z} Z^{\mu}=0 \text {. }
\end{aligned}
$$

The $Z^{\mu}$ solution describes a light-cone and will not be considered here. The $X^{\mu}$ solution has been discussed to some extent in [39]. The parameter $\mu^{2}$ sets the length scale $r^{2}$ of the space-time, while the quantum number $n$ is undetermined. In this paper, we will focus on the momentum solution

$$
Y^{\mu}=T^{\mu}
$$

The main reason for this choice is that the effective d'Alembertian $\square \equiv \square_{T}$ as well as $\left[T_{\mu}, \cdot\right]$ respect the spin $\mathcal{S}^{2}$ as discussed in section 3.1, such that the above decomposition of fluctuations into higher-spin modes is applicable. Even though the embedding is realized via the momentum generator $T^{\mu}$ rather than $X^{\mu}$, we will see that the fluctuations lead to a gauge theory on $\mathcal{M}^{3,1}$.

\section{$5 \quad$ Fluctuations and higher-spin gauge theory}

Now consider tangential ${ }^{18}$ deformations of the above background solution given by $T^{\mu}$, i.e.

$$
Y^{\mu}=T^{\mu}+\mathcal{A}^{\mu}
$$

where $\mathcal{A} \in \mathcal{C} \otimes \mathbb{C}^{4}$ is an arbitrary (Hermitian) fluctuation. We will give a complete fluctuation analysis in the following. ${ }^{19}$ The full Yang-Mills action (4.1) can be expanded in the fluctuation modes as

$$
S[Y]=S[T]+S_{2}[\mathcal{A}]+O\left(\mathcal{A}^{3}\right),
$$

where the quadratic fluctuations are governed by

$$
S_{2}[\mathcal{A}]=-\frac{2}{g^{2}} \int \mathrm{d} \Omega\left(\mathcal{A}_{\mu}\left(\mathcal{D}^{2}+\frac{1}{2} \mu^{2}\right) \mathcal{A}^{\mu}+\mathcal{G}(\mathcal{A})^{2}\right) .
$$

Here

$$
\mathcal{D}^{2} \mathcal{A}=(\square-2 \mathcal{I}) \mathcal{A}
$$

\footnotetext{
${ }^{18}$ In the 9+1-dimensional IKKT model, there are of course also transversal fluctuation modes. Those are simply scalar fields as discussed in section 3. However, it is plausible that these scalar fields acquire a non-trivial vacuum structure, along the lines of $[65,66]$ and references therein. Here we focus on the tangential modes.

${ }^{19}$ Note that the background admits $\mathrm{SO}(3,1)$ as a symmetry, which is spontaneously broken, but preserved modulo to a gauge transformation. Hence the corresponding Goldstone bosons are unphysical.
} 
is the vector Laplacian, which is composed of the scalar matrix Laplacian (or rather d'Alembertian)

$$
\square=\left[T^{\mu},\left[T_{\mu}, \cdot\right]\right] \sim-\left\{t^{\mu},\left\{t_{\mu}, .\right\}\right\}=\alpha^{-1} \square_{G}
$$

on the $\mathcal{M}^{3,1}$ background due to (2.75), and the intertwiner

$$
\mathcal{I}(\mathcal{A})^{\mu}=-\mathrm{i}\left[\left[Y^{\mu}, Y^{\nu}\right], \mathcal{A}_{\nu}\right]=\frac{\mathrm{i}}{r^{2} R^{2}}\left[\Theta^{\mu \nu}, \mathcal{A}_{\nu}\right]=:-\frac{1}{r^{2} R^{2}} \tilde{\mathcal{I}}(\mathcal{A})^{\mu},
$$

recalling $\Theta^{\mu \nu}=-r^{2} \mathcal{M}^{\mu \nu}$ as in (2.22). As usual in Yang-Mills theories, the scalar mode

$$
\mathcal{G}(\mathcal{A})=-\mathrm{i}\left[T^{\mu}, \mathcal{A}_{\mu}\right] \sim\left\{t^{\mu}, \mathcal{A}_{\mu}\right\}
$$

should be removed to get a meaningful theory. This is achieved by adding a gauge-fixing term $-\mathcal{G}(\mathcal{A})^{2}$ to the action as well as the corresponding Faddeev-Popov (or BRST) ghost. Then the quadratic action becomes

$$
S_{2}[\mathcal{A}]+S_{\text {g.f }}+S_{\text {ghost }}=-\frac{2}{g^{2}} \int \mathrm{d} \Omega\left(\mathcal{A}_{\mu}\left(\mathcal{D}^{2}+\frac{1}{2} \mu^{2}\right) \mathcal{A}^{\mu}+2 \bar{c} \square c\right)
$$

where $c$ denotes the fermionic BRST ghost; see e.g. [67] for more details.

UV spectrum and no-ghost. For short wavelengths, the quadratic action can be simplified as follows

$$
S_{2}^{\mathrm{UV}}[\mathcal{A}] \approx-\frac{2}{g^{2}} \int \mathrm{d} \Omega\left(\mathcal{A}_{\mu} \square \mathcal{A}^{\mu}+2 \bar{c} \square c\right)
$$

This has the same structure as a Yang-Mills action, which makes it very plausible that the theory will be ghost-free, as the $c$ ghost cancels the unphysical polarizations of $\mathcal{A}_{\mu}$. A simple general argument for having a ghost-free theory is as follows: ${ }^{20}$ Since the $\mathcal{A}_{0}$ component can be diagonalized using the gauge invariance, the physical, propagating degrees of freedom are carried by the remaining matrices, which are space-like such that their kinetic term has the standard sign. Indeed, we have seen that no internal higher-spin ghosts arise, due to the space-like gauge-fixing discussed above. We will provide another argument in section 5.4 that there are no ghosts, and verify to some extent how these general arguments are borne out in the mode expansion below.

\subsection{Mode expansion and ansatz}

We consider the mode expansion for vector modes similar to the case of scalar fields (3.10)

$$
\begin{aligned}
\mathcal{A}^{\mu} & =A^{\mu}(x)+A_{\alpha}^{\mu}(x) t^{\alpha}+A_{\alpha \beta}^{\mu}(x) t^{\alpha} t^{\beta}+\ldots \\
& \in \mathcal{C}^{0} \oplus \mathcal{C}^{1} \oplus \oplus \quad \mathcal{C}^{2} \oplus \ldots
\end{aligned}
$$

\footnotetext{
${ }^{20}$ we are grateful for H. Kawai for pointing this out.
} 
However these are neither irreducible nor eigenmodes of $\mathcal{D}^{2}$. To find the spin $s$ eigenmodes $\mathcal{A}_{\mu} \in \mathcal{C} \otimes \mathbb{C}^{4}$, we choose the following ansatz:

$$
\begin{aligned}
& \mathcal{A}_{\mu}^{(g)}\left[\phi^{(s)}\right]=\left\{t_{\mu}, \phi^{(s)}\right\} \quad \in \mathcal{C}^{s}, \\
& \mathcal{A}_{\mu}^{(+)}\left[\phi^{(s)}\right]=\left.\left\{x_{\mu}, \phi^{(s)}\right\}\right|_{\mathcal{C}^{s+1}} \equiv\left\{x_{\mu}, \phi^{(s)}\right\}_{+} \quad \in \mathcal{C}^{s+1}, \\
& \mathcal{A}_{\mu}^{(-)}\left[\phi^{(s)}\right]=\left.\left\{x_{\mu}, \phi^{(s)}\right\}\right|_{\mathcal{C}^{s-1}} \equiv\left\{x_{\mu}, \phi^{(s)}\right\}_{-} \quad \in \mathcal{C}^{s-1} \text {. }
\end{aligned}
$$

These expressions should be viewed as $\mathfrak{s o}(3,1)$ intertwiners

$$
\mathcal{A}^{(i)}: \quad \mathcal{C}^{s} \rightarrow \mathcal{C}^{s} \otimes \mathbb{C}^{4}, \quad i \in\{g,+,-\},
$$

where $\phi^{(s)} \in \mathcal{C}^{s}$ is used to represent the vector mode. Clearly $\mathcal{A}_{\mu}^{(g)}$ is the pure gauge mode. It is important to note that the $\mathcal{A}^{( \pm)}$intertwiners can be extended as $\mathfrak{s o}(4,1)$ intertwiners via $\mathcal{A}_{a}^{( \pm)}\left[\phi^{(s)}\right]=\left\{x_{a}, \phi^{(s)}\right\}_{ \pm}$. These are 2 linear independent modes, ${ }^{21}$ which will turn out to be exact eigenmodes of $\mathcal{D}^{2}$. For completeness, we need one more such mode. We will use the ansatz

$$
\mathcal{A}_{\mu}^{(\tau)}\left[\phi^{(s)}\right]=x_{\mu} \phi^{(s)}
$$

as a starting point, because it is independent of the above modes and it also extends as $\mathfrak{s o}(4,1)$ intertwiner $\mathcal{A}_{a}^{(\tau)}[\phi]=x_{a} \phi$. This is a time-like mode, but we will argue that it is not part of the physical Hilbert space of the theory.

\subsection{Group-theoretical considerations}

To evaluate $\mathcal{D}^{2}$ on these modes, we need some group-theoretical preparations.

Intertwiners. Consider the following $\mathrm{SO}(3,1)$ intertwiners

$$
\begin{aligned}
\mathcal{C} \otimes \mathbb{C}^{4} & \rightarrow \mathcal{C} \otimes \mathbb{C}^{4} \\
\mathcal{A}^{\mu} & \mapsto \tilde{\mathcal{I}}(\mathcal{A})^{\mu}=-\mathrm{i}\left[\Theta^{\mu \nu}, \mathcal{A}_{\nu}\right] \sim\left\{\theta^{\mu \nu}, \mathcal{A}_{\nu}\right\} \\
\mathcal{A}^{\mu} & \mapsto \square(\mathcal{A})_{\mu}=\left[T^{\nu},\left[T_{\nu}, \mathcal{A}_{\mu}\right]\right] \sim-\left\{t^{\nu},\left\{t_{\nu}, \mathcal{A}_{\mu}\right\}\right\}
\end{aligned}
$$

which are Hermitian, in the sense

$$
\int \mathrm{d} \Omega \mathcal{A}_{\mu} \tilde{\mathcal{I}}\left(\mathcal{A}^{\prime}\right)^{\mu}=\int \mathrm{d} \Omega \tilde{\mathcal{I}}(\mathcal{A})_{\mu} \mathcal{A}^{\prime \mu}
$$

and similarly for $\square$. We recall that $\mathcal{S}^{2}$ commutes with $\square$, see (3.5), and it also commutes with $\tilde{\mathcal{I}}$. This will greatly facilitate the analysis. Note that the gauge-fixing functional $\mathcal{G}$ is also an $\mathrm{SO}(3,1)$ intertwiner.

\footnotetext{
${ }^{21}$ We note that $\mathcal{A}_{\mu}^{(-)}=-\alpha_{s}\left(\square_{H}-2 r^{2}\right) \phi_{\mu}^{(s)}$ in the notation of [40]. But the notation $\phi_{a}^{(s)}$ is inconsistent with the present, hence we avoid it here.
} 
Relation with Casimirs. Recall from (3.5) the Laplacian

$$
\square=\left[T_{\mu},\left[T^{\mu}, \cdot\right]\right]=R^{-2}\left(C^{2}[\mathfrak{s o}(4,1)]^{(\mathrm{ad})}-C^{2}[\mathfrak{s o}(3,1)]^{(\mathrm{ad})}\right),
$$

where $(\mathrm{ad})$ denotes the representation of $\mathfrak{s o}(4,1)$ or $\mathfrak{s o}(3,1)$ acting on $\mathcal{C}$ via $M_{\mu \nu}^{(\mathrm{ad})}=$ $\left[\mathcal{M}_{\mu \nu}, \cdot\right] \sim \mathrm{i}\left\{\mathcal{M}_{\mu \nu}, \cdot\right\}$. Then $\mathcal{A}_{a}$ transforms in (full) $=(\mathrm{ad}) \otimes(5)$ of $\mathrm{SO}(4,1)$. Now consider the intertwiners

$$
\begin{aligned}
& \tilde{\mathcal{I}}^{(5)}[\mathcal{A}]^{a}:=-\mathrm{i}\left[\Theta^{a b}, \mathcal{A}_{b}\right] \sim\left\{\theta^{a b}, \mathcal{A}_{b}\right\} \\
& \tilde{\mathcal{I}}^{(4)}[\mathcal{A}]^{\mu}=-\mathrm{i}\left[\Theta^{\mu \nu}, \mathcal{A}_{\nu}\right] \sim\left\{\theta^{\mu \nu}, \mathcal{A}_{\nu}\right\}
\end{aligned}
$$

which arise in $\mathcal{D}^{2}$. They can be related to the Casimir $C^{2}[\mathfrak{s o}(4,1)]^{(\text {full })}$ acting on vector modes as follows:

$$
\begin{aligned}
C^{2}[\mathfrak{s o}(4,1)]^{(\mathrm{full})} \mathcal{A}^{a} & =\frac{1}{2}\left(\left[\mathcal{M}_{c d}, \cdot\right]+M_{c d}^{(5)}\right)^{2} \mathcal{A}^{a} \\
& =\left(C^{2}[\mathfrak{s o}(4,1)]^{(\mathrm{ad})}-2 r^{-2} \tilde{\mathcal{I}}^{(5)}+4\right) \mathcal{A}^{a}
\end{aligned}
$$

using $(3.2)$, and $C^{2}[\mathfrak{s o}(4,1)]^{(5)}=4$ for the vector representation $\mathbb{C}^{5}$. This can be seen by expressing $\tilde{\mathcal{I}}$ as follows:

$$
-r^{2}\left(M_{c d}^{(\mathrm{ad})} \otimes M_{c d}^{(5)} \mathcal{A}\right)^{a} \sim-\left(M_{c d}^{(5)}\right)_{b}^{a} \mathrm{i}\left\{\theta^{c d}, \cdot\right\} \mathcal{A}^{b}=2\left\{\theta_{a b}, \mathcal{A}^{b}\right\}=2 \tilde{\mathcal{I}}^{(5)}(\mathcal{A})^{a}
$$

where

$$
\left(M_{a b}^{(5)}\right)_{d}^{c}=\mathrm{i}\left(\delta_{b}^{c} \eta_{a d}-\delta_{a}^{c} \eta_{b d}\right)
$$

is the $\mathfrak{s o}(4,1)$ vector representation. Thus

$$
\begin{aligned}
& 2 r^{-2} \tilde{\mathcal{I}}^{(5)}=-C^{2}[\mathfrak{s o}(4,1)]^{(5) \otimes(\mathrm{ad})}+C^{2}[\mathfrak{s o}(4,1)]^{(\mathrm{ad})}+C^{2}[\mathfrak{s o}(4,1)]^{(5)} \\
& 2 r^{-2} \tilde{\mathcal{I}}^{(4)}=-C^{2}[\mathfrak{s o}(3,1)]^{(4) \otimes(\mathrm{ad})}+C^{2}[\mathfrak{s o}(3,1)]^{(\mathrm{ad})}+C^{2}[\mathfrak{s o}(3,1)]^{(4)}
\end{aligned}
$$

cf. [40], where $C^{2}[\mathfrak{s o}(3,1)]^{(4)}=3$. Using (5.16), we can rewrite (5.18) and its $\mathfrak{s o}(3,1)$ analog as

$$
\begin{aligned}
C^{2}[\mathfrak{s o}(4,1)]^{(\mathrm{full})} \mathcal{A}^{a} & =\left(C^{2}[\mathfrak{s o}(4,1)]^{(\mathrm{ad})}-2 r^{-2} \tilde{\mathcal{I}}^{(5)}+4\right) \mathcal{A}^{a} \\
& =\left(R^{2} \square+C^{2}[\mathfrak{s o}(3,1)]^{(\mathrm{ad})}-2 r^{-2} \tilde{\mathcal{I}}^{(5)}+4\right) \mathcal{A}^{a} \\
& =\mathcal{A}^{a}\left[C^{2}[\mathfrak{s o}(4,1)] \phi\right], \\
C^{2}[\mathfrak{s o}(3,1)]^{(\mathrm{fulll})} \mathcal{A}^{\mu} & =\left(C^{2}[\mathfrak{s o}(3,1)]^{(\mathrm{ad})}-2 r^{-2} \tilde{\mathcal{I}}^{(4)}+3\right) \mathcal{A}^{\mu} \\
& =\mathcal{A}^{a}\left[C^{2}[\mathfrak{s o}(3,1)] \phi\right],
\end{aligned}
$$

assuming that $\mathcal{A}^{a}[\phi]$ is an $\mathfrak{s o}(4,1)$ intertwiner such as (5.11) and (5.13). Subtracting the right-hand-side of (5.23) from (5.22) for $a=\mu$, we obtain

$$
\begin{aligned}
\left(R^{2} \square-2 r^{-2}\left(\tilde{\mathcal{I}}^{(5)}-\tilde{\mathcal{I}}^{(4)}\right)+1\right) \mathcal{A}^{\mu} & =\mathcal{A}^{\mu}\left[\left(C^{2}[\mathfrak{s o}(4,1)]-C^{2}[\mathfrak{s o}(3,1)]\right) \phi\right] \\
& =R^{2} \mathcal{A}^{\mu}[\square \phi]
\end{aligned}
$$


which gives

$$
\mathcal{D}^{2} \mathcal{A}^{\mu}=\left(\square+\frac{2}{r^{2} R^{2}} \tilde{\mathcal{I}}^{(4)}\right) \mathcal{A}^{\mu}=\mathcal{A}^{\mu}\left[\left(\square-\frac{1}{R^{2}}\right) \phi\right]+\frac{2}{r^{2} R^{2}} \tilde{\mathcal{I}}^{(5)} \mathcal{A}^{\mu} .
$$

This can now be evaluated using the results on $H^{4}$ in [40], and we obtain an eigenmode of $\mathcal{D}^{2}$ if $\mathcal{A}^{a}[\phi]$ is an eigenmode of $\tilde{\mathcal{I}}^{(5)}$. Finally, we remark that all relations written in the semi-classical (Poisson) case generalize to the fully noncommutative case.

\section{$5.3 \quad \mathcal{D}^{2}$ eigenvalues}

Consider first the pure gauge mode $\mathcal{A}_{\mu}^{(g)}[\phi]=\left\{t_{\mu}, \phi\right\}$ (5.11), which define a flat direction for the gauge-invariant action (5.3). This means that

$$
\left(\mathcal{D}^{2}+\frac{1}{2} \mu^{2}\right) \mathcal{A}_{\mu}^{(g)}+\left\{t_{\mu},\left\{t^{\nu}, \mathcal{A}_{\nu}^{(g)}\right\}\right\}=0
$$

and therefore

$$
\mathcal{D}^{2} \mathcal{A}_{\mu}^{(g)}[\phi]=\mathcal{A}_{\mu}^{(g)}\left[\left(\square+\frac{3}{R^{2}}\right) \phi\right]
$$

using $\frac{1}{2} \mu^{2}=-3 R^{-2}$ (4.7). Alternatively, relation (5.27) can be computed directly as a consistency check. Next, consider the $\mathcal{A}_{\mu}^{( \pm)}$modes. Since they are part of the $\mathrm{SO}(4,1)$ covariant modes $\left\{x_{a}, \phi\right\}_{ \pm}$, we can use (5.25) and (A.31) to obtain

$$
\begin{aligned}
\mathcal{D}^{2} \mathcal{A}_{\mu}^{(+)}\left[\phi^{(s)}\right] & =\mathcal{A}_{\mu}^{(+)}\left[\left(\square-\frac{1}{R^{2}}\right) \phi\right]+\frac{2}{R^{2}}(s+3)\left\{x^{\mu}, \phi^{(s)}\right\}_{+} \\
& =\mathcal{A}_{\mu}^{(+)}\left[\left(\square+\frac{2 s+5}{R^{2}}\right) \phi^{(s)}\right], \\
\mathcal{D}^{2} \mathcal{A}_{\mu}^{(-)}\left[\phi^{(s)}\right] & =\mathcal{A}_{\mu}^{(-)}\left[\left(\square+\frac{-2 s+3}{R^{2}}\right) \phi^{(s)}\right] .
\end{aligned}
$$

Defining $\operatorname{End}\left(\mathcal{H}_{n}\right)$ to be the (Hilbert) space of Hilbert-Schmidt operators as in (3.1), it decomposes into unitary irreps of $\mathfrak{s o}(4,2)$, which can be decomposed further into eigenmodes of $\square$ since the latter is expressed in terms of Casimirs (3.3). Thus diagonalizing $\square$ on $\mathcal{C}^{s}$ we obtain three series of eigenmodes of $\mathcal{D}^{2}$, and in particular three series of on-shell modes which satisfy $\left(\mathcal{D}^{2}-\frac{3}{R^{2}}\right) \mathcal{A}=0$ :

$$
\begin{aligned}
\mathcal{A}^{(+)}\left[\phi^{(s)}\right] & \text { for } & \left(\square+\frac{2 s+2}{R^{2}}\right) \phi^{(s)} & =0, \\
\mathcal{A}^{(-)}\left[\phi^{(s)}\right] & \text { for } & \left(\square+\frac{-2 s}{R^{2}}\right) \phi^{(s)} & =0 \\
\mathcal{A}^{(g)}\left[\phi^{(s)}\right] & \text { for } & \square \phi^{(s)} & =0 .
\end{aligned}
$$

Of course the pure gauge mode $\mathcal{A}^{(g)}$ is unphysical.

Finally, the mode $\mathcal{A}_{\mu}^{(\tau)}$ is also part of an $\mathrm{SO}(4,1)$ mode $\mathcal{A}_{a}^{(\tau)}[\phi]=x_{a} \phi$. Thus formula (5.25) gives

$$
\mathcal{D}^{2} \mathcal{A}_{\mu}^{(\tau)}[\phi]=\mathcal{A}_{\mu}^{(\tau)}\left[\left(\square-\frac{1}{R^{2}}\right) \phi\right]+\frac{2}{r^{2} R^{2}} \tilde{\mathcal{I}}^{(5)} \mathcal{A}_{\mu}^{(\tau)}[\phi]
$$


but now $\tilde{\mathcal{I}}^{(5)}$ is no longer diagonal:

$$
\begin{aligned}
\tilde{\mathcal{I}}^{(5)} \mathcal{A}_{\mu}^{(\tau)}[\phi] & =\left\{\theta^{\mu b}, x_{b} \phi\right\}=\left\{\theta^{\mu b}, x_{b}\right\} \phi+x_{b}\left\{\theta^{\mu b}, \phi\right\} \\
& =4 r^{2} x_{\mu} \phi-\theta^{\mu b}\left\{x_{b}, \phi\right\} \\
& =4 r^{2} \mathcal{A}_{\mu}^{(\tau)}[\phi]+r^{2} R^{2 \check{\partial}^{\mu} \phi}
\end{aligned}
$$

where

$$
\check{\partial}^{a} \phi^{(s)}:=-\frac{1}{r^{2} R^{2}} \theta^{a b}\left\{x_{b}, \phi\right\} \quad \in \mathcal{C}^{s}
$$

is the tangential derivative operator on $H^{4}$ introduced in [40]. Thus, we arrive at

$$
\mathcal{D}^{2} \mathcal{A}_{\mu}^{(\tau)}[\phi]=\mathcal{A}_{\mu}^{(\tau)}\left[\left(\square+\frac{7}{R^{2}}\right) \phi\right]+2 \widetilde{\partial}_{\mu} \phi
$$

Degeneracy. We can recognize a degeneracy of these modes by considering the eigenmodes in the same $\mathcal{C}^{s}$. Then the above results give

$$
\begin{aligned}
\mathcal{D}^{2} \mathcal{A}_{\mu}^{(+)}\left[\phi^{(s-1)}\right] & =\mathcal{A}_{\mu}^{(+)}\left[\left(\square+\frac{2 s+3}{R^{2}}\right) \phi^{(s-1)}\right] \quad \in \mathcal{C}^{s}, \\
\mathcal{D}^{2} \mathcal{A}_{\mu}^{(-)}\left[\phi^{(s+1)}\right] & =\mathcal{A}_{\mu}^{(-)}\left[\left(\square+\frac{-2 s+1}{R^{2}}\right) \phi^{(s+1)}\right] \quad \in \mathcal{C}^{s}, \\
\mathcal{D}^{2} \mathcal{A}_{\mu}^{(g)}\left[\phi^{(s)}\right] & =\mathcal{A}_{\mu}^{(g)}\left[\left(\square+\frac{3}{R^{2}}\right) \phi^{(s)}\right] \quad \in \mathcal{C}^{s} .
\end{aligned}
$$

Now recall that $D^{ \pm}: \mathcal{C}^{s} \rightarrow \mathcal{C}^{s \pm 1}$ relates eigenfunctions and eigenvalues according to (3.16)(3.20). Therefore

$$
\begin{aligned}
& \left(\mathcal{D}^{2}+\frac{1}{2} \mu^{2}\right) \mathcal{A}_{\mu}^{(+)}\left[D^{-} \phi^{(s)}\right]=\mathcal{A}_{\mu}^{(+)}\left[\left(\square+\frac{2 s}{R^{2}}\right) D^{-} \phi^{(s)}\right]=\mathcal{A}_{\mu}^{(+)}\left[D^{-} \square \phi^{(s)}\right], \\
& \left(\mathcal{D}^{2}+\frac{1}{2} \mu^{2}\right) \mathcal{A}_{\mu}^{(-)}\left[D^{+} \phi^{(s)}\right]=\mathcal{A}_{\mu}^{(-)}\left[\left(\square+\frac{-2 s-2}{R^{2}}\right) D^{+} \phi^{(s)}\right]=\mathcal{A}_{\mu}^{(-)}\left[D^{+} \square \phi^{(s)}\right],
\end{aligned}
$$

using (A.38). Hence if $\square \phi^{(s)}=m^{2} \phi^{(s)}$ is an eigenmode, we obtain 3 degenerate eigenmodes of $\mathcal{D}^{2}$ with the same eigenvalue

$$
\begin{aligned}
\left(\mathcal{D}^{2}+\frac{1}{2} \mu^{2}\right) \mathcal{A}_{\mu}^{(+)}\left[D^{-} \phi^{(s)}\right] & =m^{2} \mathcal{A}_{\mu}^{(+)}\left[D^{-} \phi^{(s)}\right] \\
\left(\mathcal{D}^{2}+\frac{1}{2} \mu^{2}\right) \mathcal{A}_{\mu}^{(-)}\left[D^{+} \phi^{(s)}\right] & =m^{2} \mathcal{A}_{\mu}^{(-)}\left[D^{+} \phi^{(s)}\right] \\
\left(\mathcal{D}^{2}+\frac{1}{2} \mu^{2}\right) \mathcal{A}_{\mu}^{(g)}\left[\phi^{(s)}\right] & =m^{2} \mathcal{A}_{\mu}^{(g)}\left[\phi^{(s)}\right] .
\end{aligned}
$$

Whether or not these modes are always linearly independent is not yet established. This could be decided using the inner products (5.58i). A non-trivial consistency check is provided in appendix A.5, using the action of $\tilde{\mathcal{I}}$ (A.33):

$$
\begin{aligned}
& \tilde{\mathcal{I}} \mathcal{A}_{\mu}^{(-)}\left[D^{+} \phi^{(s)}\right]=r^{2}(-s+1) \mathcal{A}^{\mu(-)}\left[D^{+} \phi^{(s)}\right]+r^{2} R\left\{t^{\mu}, D^{-} D^{+} \phi^{(s)}\right\}, \\
& \tilde{\mathcal{I}} \mathcal{A}_{\mu}^{(+)}\left[D^{-} \phi^{(s)}\right]=r^{2}(s+2) \mathcal{A}^{\mu(+)}\left[D^{-} \phi^{(s)}\right]+r^{2} R\left\{t^{\mu}, D^{+} D^{-} \phi^{(s)}\right\} .
\end{aligned}
$$




\subsection{Gauge fixing and physical Hilbert space}

As always in Yang-Mills gauge theory, the fluctuations $\mathcal{A}^{\mu}$ can be separated into gauge-fixed modes denoted as $\mathcal{B}^{\mu}$ and an unphysical scalar mode $\chi$, which should be determined via

$$
\begin{aligned}
\mathcal{B}^{\mu} & =\mathcal{A}^{\mu}+\mathcal{A}_{(g)}^{\mu}[\chi], \\
0 & =\mathcal{G}(\mathcal{B}) \equiv\left\{t^{\mu}, \mathcal{B}_{\mu}\right\}=\left\{t^{\mu}, \mathcal{A}_{\mu}\right\}-\square \chi .
\end{aligned}
$$

Then the equations of motion for $\mathcal{B}^{\mu}$ become

$$
\begin{aligned}
\left(\mathcal{D}^{2}+\frac{1}{2} \mu^{2}\right) \mathcal{B}_{\mu} & =\left(\mathcal{D}^{2}+\frac{1}{2} \mu^{2}\right) \mathcal{A}_{\mu}+\left(\mathcal{D}^{2}+\frac{1}{2} \mu^{2}\right)\left\{t_{\mu}, \chi\right\} \\
& =\left(\mathcal{D}^{2}+\frac{1}{2} \mu^{2}\right) \mathcal{A}_{\mu}+\left\{t_{\mu}, \square \chi\right\} \\
& =\left(\mathcal{D}^{2}+\frac{1}{2} \mu^{2}\right) \mathcal{A}_{\mu}+\left\{t_{\mu},\left\{t^{\nu}, \mathcal{A}_{\nu}\right\}\right\}
\end{aligned}
$$

using (5.26). Hence $\mathcal{A}_{\mu}$ is a solution of the non-gauge-fixed action (5.3) if and only if $\mathcal{B}_{\mu}$ is a solution $\left(\mathcal{D}^{2}+\frac{1}{2} \mu^{2}\right) \mathcal{B}_{\mu}=0$ of the gauge-fixed action. ${ }^{22} \chi$ is determined only up to the kernel of $\square$, which must be factored out in the physical Hilbert space.

Let us make this explicit for the above modes. The following relations (A.35) are shown in appendix A.4

$$
\begin{aligned}
\left\{t^{\mu}, \mathcal{A}_{\mu}^{(+)}\left[\phi^{(s)}\right]\right\} & =\frac{s+3}{R} D^{+} \phi^{(s)}, \\
\left\{t^{\mu}, \mathcal{A}_{\mu}^{(-)}\left[\phi^{(s)}\right]\right\} & =\frac{-s+2}{R} D^{-} \phi^{(s)} .
\end{aligned}
$$

Hence in the divergence-free sector and for $s=2, \mathcal{A}_{\mu}^{(-)}$is already gauge fixed. For the time-like mode $\mathcal{A}_{\mu}^{(\tau)}=x_{\mu} \phi^{(s)}$, we obtain

$$
\left\{t^{\mu}, \mathcal{A}_{\mu}^{(\tau)}\left[\phi^{(s)}\right]\right\}=\left\{t^{\mu}, x_{\mu} \phi^{(s)}\right\}=\sinh (\eta)(4+s+\tau) \phi^{(s)},
$$

using (3.41). We will argue below that the $\mathcal{A}_{\mu}^{(\tau)}$ modes do not contribute to the physical Hilbert space. Finally, the pure gauge mode satisfies

$$
\left\{t^{\mu}, \mathcal{A}_{\mu}^{(g)}[\phi]\right\}=-\square \phi,
$$

which vanishes for on-shell pure gauge fields. Together with (A.38), this allows to determine the $\mathcal{B}_{\mu}^{( \pm)}$explicitly:

$$
\begin{aligned}
& \chi_{(+)}=\frac{s+3}{R} \square^{-1} D^{+} \phi^{(s)}=\frac{s+3}{R} D^{+}\left(\left(\square+\frac{2 s+2}{R^{2}}\right)^{-1} \phi^{(s)}\right), \\
& \chi_{(-)}=\frac{2-s}{R} \square^{-1} D^{-} \phi^{(s)}=\frac{2-s}{R} D^{-}\left(\left(\square-\frac{2 s}{R^{2}}\right)^{-1} \phi^{(s)}\right) .
\end{aligned}
$$

\footnotetext{
${ }^{22}$ In the presence of matter, this generalizes as $\left(\mathcal{D}^{2}+\frac{1}{2} \mu^{2}\right) \mathcal{B}_{\mu}=\mathcal{J}_{\mu}$, where $\mathcal{J}_{\mu}$ is the conserved current.
} 
Assuming that these expressions make sense, it follows that the gauge-fixed modes satisfy the intertwiner relations

$$
\begin{aligned}
\mathcal{D}^{2} \mathcal{B}_{\mu}^{(+)}\left[\phi^{(s)}\right] & =\mathcal{A}_{\mu}^{(+)}\left[\left(\square+\frac{2 s+5}{R^{2}}\right) \phi^{(s)}\right]+\frac{s+3}{R}\left\{t_{\mu},\left(\square+\frac{3}{R^{2}}\right) \square^{-1} D^{+} \phi^{(s)}\right\} \\
& =\mathcal{B}_{\mu}^{(+)}\left[\left(\square+\frac{2 s+5}{R^{2}}\right) \phi^{(s)}\right], \\
\mathcal{D}^{2} \mathcal{B}_{\mu}^{(-)}\left[\phi^{(s)}\right] & =\mathcal{B}_{\mu}^{(-)}\left[\left(\square+\frac{-2 s+3}{R^{2}}\right) \phi^{(s)}\right]
\end{aligned}
$$

using (5.27) and (5.45). Hence they are eigenmodes of $\mathcal{D}^{2}$ if the underlying modes $\phi^{(s)}$ are eigenmodes of $\square$. In particular,

$$
\begin{aligned}
\left(\mathcal{D}^{2}+\frac{1}{2} \mu^{2}\right) \mathcal{B}_{\mu}^{(+)}\left[D^{-} \phi^{(s)}\right] & =\mathcal{B}_{\mu}^{(+)}\left[D^{-} \square \phi^{(s)}\right], \\
\left(\mathcal{D}^{2}+\frac{1}{2} \mu^{2}\right) \mathcal{B}_{\mu}^{(-)}\left[D^{+} \phi^{(s)}\right] & =\mathcal{B}_{\mu}^{(-)}\left[D^{+} \square \phi^{(s)}\right], \\
\left(\mathcal{D}^{2}+\frac{1}{2} \mu^{2}\right) \mathcal{A}_{\mu}^{(g)}\left[\phi^{(s)}\right] & =\mathcal{A}_{\mu}^{(g)}\left[\square \phi^{(s)}\right],
\end{aligned}
$$

cf. (5.37). We observe again the triple degeneracy of $\mathcal{D}^{2}$, unless some modes coincide or vanish.

Physical Hilbert space. Now consider (5.45) in more detail. Comparing with (5.30), we see that these expressions are well-defined for off-shell modes, but not for on-shell modes. Indeed if $\mathcal{A}$ is on-shell, the added pure gauge term must also be on-shell. But this means by (5.44) that it is gauge-fixed, so that it cannot change the gauge of $\mathcal{A}$. Hence on-shell modes cannot simply be gauge-fixed, and the physical Hilbert space contains only those on-shell modes which are gauge-fixed (modulo pure gauge modes). Due to the degeneracy (5.47), we can always find a linear combination

$$
\mathcal{A}^{(p h)}:=\mathcal{A}^{(-)}\left[\phi_{+}^{(s+1)}\right]+\mathcal{A}^{(+)}\left[\phi_{-}^{(s-1)}\right] \quad \in \mathcal{C}^{s}, \quad\left(\mathcal{D}^{2}+\frac{1}{2} \mu^{2}\right) \mathcal{A}^{(p h)}=0
$$

of on-shell modes with

$$
\left(\square+\frac{2 s}{R^{2}}\right) \phi_{-}^{(s-1)}=0=\left(\square-\frac{2 s+2}{R^{2}}\right) \phi_{+}^{(s+1)}
$$

which is gauge-fixed, i.e.

$$
0=\left\{t^{\mu}, \mathcal{A}_{\mu}^{(p h)}\right\}=\frac{-s+1}{R} D^{-} \phi_{+}^{(s+1)}+\frac{s+2}{R} D^{+} \phi_{-}^{(s-1)} .
$$

Here we have to distingish generic $s$ and $s=1$. For $s=1$, the physical solutions are

$$
\mathcal{A}^{(p h-)}\left[\phi^{(2)}\right]:=\mathcal{A}^{(-)}\left[\phi^{(2)}\right], \quad\left(\square-\frac{4}{R^{2}}\right) \phi^{(2)}=0
$$

while none of the $\mathcal{A}^{(+)}\left[\phi_{-}^{(0)}\right]$ is physical. For $s \neq 1, \phi_{+}^{(s+1)}$ is uniquely determined by the above equation as

$$
\phi_{+}^{(s+1)}=\frac{s+3}{s-1}\left(D^{+} D^{-}\right)^{-1} D^{+} D^{+} \phi_{-}^{(s-1)}, \quad\left(\square+\frac{2 s}{R^{2}}\right) \phi_{-}^{(s-1)}=0
$$


Note that $D^{+} D^{-}=D^{+}\left(D^{+}\right)^{\dagger}$ commutes with $\square$ due to (A.38) and is positive semi-definite, and vanishes only on $\phi^{(s, 0)}$. Therefore the inverse in (5.52) exists, and the relation is compatible with the on-shell conditions. Explicitly, this gives the following physical solutions

$$
\begin{aligned}
\mathcal{A}^{(p h-)}\left[\phi^{(2)}\right] & :=\mathcal{A}^{(-)}\left[\phi^{(2)}\right] \\
\mathcal{A}^{(p h-)}\left[\phi^{(s+1,0)}\right] & :=\mathcal{A}^{(-)}\left[\phi^{(s+1,0)}\right], \\
\mathcal{A}^{(p h)}\left[\phi^{(s-1)}\right] & :=\mathcal{A}^{(+)}\left[\phi^{(s-1)}\right]+\frac{s+3}{s-1} \mathcal{A}^{(-)}\left[\left(D^{+} D^{-}\right)^{-1} D^{+} D^{+} \phi^{(s-1)}\right], s>1 .
\end{aligned}
$$

The lowest physical modes are

$$
\left\{\mathcal{A}^{(-)}\left[\phi^{(0)}\right], \mathcal{A}^{(-)}\left[\phi^{(1,0)}\right], \mathcal{A}^{(-)}\left[\phi^{(2)}\right], \mathcal{A}^{(+)}\left[\phi^{(1)}\right]+5 \mathcal{A}^{(-)}\left[\left(D^{+} D^{-}\right)^{-1} D^{+} D^{+} \phi^{(1)}\right], \ldots\right\}
$$

The physical Hilbert space $\mathcal{H}_{\text {phys }}$ of the linearized theory, therefore, consists of the on-shell modes

$$
\begin{array}{llrlrl}
\mathcal{A}^{(p h)}\left[\phi^{(s-1)}\right] & \text { for } & \left(\square+\frac{2 s}{R^{2}}\right) \phi^{(s-1)} & =0, & s \geq 2 \\
\mathcal{A}^{(p h-)}\left[\phi^{(s+1,0)}\right] & \text { for } & \left(\square-\frac{2 s+2}{R^{2}}\right) \phi^{(s+1,0)} & =0, & s \geq 0 \\
\mathcal{A}^{(p h-)}\left[\phi^{(2)}\right] & \text { for } & \left(\square-\frac{4}{R^{2}}\right) \phi^{(2)}=0 &
\end{array}
$$

which satisfy $\left(\mathcal{D}^{2}+\frac{1}{2} \mu^{2}\right) \mathcal{A}^{(p h)}=0$. The on-shell pure gauge solutions

$$
\mathcal{A}^{(g)}\left[\phi^{(s)}\right] \quad \text { for } \square \phi^{(s)}=0
$$

are null (see section 5.5) and must be factored out from $\mathcal{H}_{\text {phys }}$. Finally, we argue in appendix A.3 that there are no on-shell gauge-fixed solutions involving $\mathcal{A}_{\mu}^{(\tau)}[\phi]$. To summarize,

$$
\mathcal{H}_{\text {phys }}=\left\{\mathcal{A}^{(p h)}\left[\phi^{(s-1)}\right], s=2,3, \ldots n\right\} \cup\left\{\mathcal{A}^{(p h-)}\left[\phi^{(s+1,0)}\right], s=0,1, \ldots n\right\} \cup\left\{\mathcal{A}^{(p h-)}\left[\phi^{(2)}\right]\right\}
$$

subject to the on-shell conditions (5.55). Here we indicate also the cutoff $n$, which disappears in the semi-classical limit $n \rightarrow \infty$. Since $\square$ encodes the effective metric $G_{\mu \nu}$, the propagation of these modes respects local Lorentz-invariance, and the extra structures $\tau$ and $\kappa^{\mu \nu}$ do not enter. We conjecture that this list is complete, although this has not been shown. However off-shell, a $4^{\text {th }}$ series of modes is clearly missing, which is needed e.g. for the propagator.

In view of the discussion on space-like gauge in section 3.3, the $\phi^{(s, 0)}$ modes should be interpreted as massless spin $s$ fields, while the full $\phi^{(s)}$ modes are interpreted as (would-be) massive spin $s$ fields. Thus we have found one massless and one massive tower of spin $s$ fields, as well as a would-be massive spin 2 field. The term "would-be massive" fields indicates the degrees of freedom of massive fields but without explicit mass term. The physical significance of these modes needs further investigation. For example, the pure gauge modes $\mathcal{A}^{(g)}$ may mix or even coincide with some of the would-be massive gauge fields, which could make them massless or partially massless. Off-shell, the $\mathcal{B}^{ \pm}$provide the degrees of freedom of two massive spin $s$ fields. 


\subsection{Inner products and quadratic action}

We have argued following the quadratic action (5.3) that the matrix model should lead to a ghost-free theory, based on rather general arguments. To see this explicitly and to clarify the on-shell Hilbert space structure, we elaborate the inner product matrix for the fluctuation modes $\mathcal{A}$ introduced above. This inner products is also needed to compute the propagator. To simplify the notation we set $\int \equiv \int \mathrm{d} \Omega$. Then using (3.41), (3.48), (3.49), (3.50) and (A.35), we obtain

$$
\begin{aligned}
\int \mathcal{A}_{\mu}^{(g)}\left[\phi^{\prime}\right] \mathcal{A}^{(g) \mu}[\phi] & =\int \phi^{\prime} \square \phi \\
\int \mathcal{A}_{\mu}^{(g)}\left[\phi^{\prime\left(s^{\prime}\right)}\right] \mathcal{A}^{(+) \mu}\left[\phi^{(s)}\right] & =-\frac{s+3}{R} \int_{\mathcal{M}^{3,1}} \phi^{\prime\left(s^{\prime}\right)} D^{+} \phi^{(s)} \\
\int \mathcal{A}_{\mu}^{(g)}\left[\phi^{\prime\left(s^{\prime}\right)}\right] \mathcal{A}^{(-) \mu}\left[\phi^{(s)}\right] & =\frac{s-2}{R} \int \phi^{\prime\left(s^{\prime}\right)} D^{-} \phi^{(s)} \\
\int \mathcal{A}_{\mu}^{(-)}\left[\phi^{\prime}\right] \mathcal{A}^{(+) \mu}[\phi] & =\int D^{-} D^{-} \phi^{\prime} \phi \\
\int \mathcal{A}_{\mu}^{(+)}\left[\phi^{\prime\left(s^{\prime}\right)}\right] \mathcal{A}^{(+) \mu}\left[\phi^{(s)}\right] & =\int \phi^{\prime\left(s^{\prime}\right)}\left(\left(1-\alpha_{s}\right) \square_{H}+2 \alpha_{s} r^{2}(s+1)+D^{-} D^{+}\right) \phi^{(s)} \\
\int \mathcal{A}_{\mu}^{(-)}\left[\phi^{\prime\left(s^{\prime}\right)}\right] \mathcal{A}^{(-) \mu}\left[\phi^{(s)}\right] & =\int \phi^{\prime\left(s^{\prime}\right)}\left(\alpha_{s}\left(\square_{H}-2 r^{2}(s+1)\right)+D^{+} D^{-}\right) \phi^{(s)} \\
\int \mathcal{A}_{\mu}^{(\tau)}\left[\phi^{\prime}\right] \mathcal{A}^{( \pm) \mu}[\phi] & =-\int \phi^{\prime} x_{4} D^{ \pm} \phi \\
\int \mathcal{A}_{\mu}^{(\tau)}\left[\phi^{\prime}\right] \mathcal{A}^{(g) \mu}[\phi] & =\int \frac{x_{4}}{R} \phi^{\prime\left(s^{\prime}\right)}(s+\tau) \phi^{(s)} \\
\int \mathcal{A}_{\mu}^{(\tau)}\left[\phi^{\prime}\right] \mathcal{A}^{(\tau) \mu}[\phi] & =-R^{2} \int \cosh ^{2}(\eta) \phi^{\prime} \phi
\end{aligned}
$$

Note that the on-shell pure gauge modes $\mathcal{A}^{(g)}[\phi]$ with $\square \phi=0$ are null, but these are factored out from $\mathcal{H}_{\text {phys }}$. Observe also the negative sign of the time-like $\mathcal{A}^{(\tau)}$ mode, which couples to $\mathcal{A}^{(g)}$.

Now consider the physical sector $\mathcal{H}_{\text {phys }}$. Since the null modes $\mathcal{A}^{(g)}$ are orthogonal to all gauge-fixed modes, these must all be positive, because the 4 -vectors $\mathcal{A}^{\mu}$ have only one negative and three positive components at each point of $\mathcal{M}^{3,1}$. Here we use the fact that $\mathcal{C}^{s}$ has a positive definite inner product (3.1), which is related to the space-like gauge as discussed in section 3.2. Hence the theory is ghost-free, as expected. ${ }^{23}$

We can also check this explicitly for some modes. The positivity of $\left(\square_{H}-2 r^{2}(s+1)\right)$, see (3.46), implies that the inner product of the $\mathcal{A}^{(-)}\left[\phi^{(s, 0)}\right]$ is indeed positive. This follows also from $x^{\mu} \mathcal{A}_{\mu}^{(-)}\left[\phi^{(s, 0)}\right]=0$, which means that the time-like component vanishes. Furthermore, all the $\mathcal{A}^{(-)}\left[\phi^{(2)}\right]$ modes have positive norm. These are the modes which will contribute to gravity.

\footnotetext{
${ }^{23}$ It is quite remarkable that this is possible, in spite of having only 3 rather than 4 diffeomorphism degrees of freedom. The underlying reason is the reduced Lorentz invariance, which enables the internal space-like gauge (3.12).
} 
Discussion. The physical significance of these modes is most transparent in space-like gauge. This suggests to interpret the divergence-free modes $\mathcal{A}^{(-)}\left[\phi^{(s, 0)}\right]$ as massless spin $s$ gauge fields, while the generic modes $\mathcal{A}^{(p h)}\left[\phi^{(s)}\right]$ describe would-be massive spin $s$ fields, according to the discussion in section 3.4. Indeed we will see in the case of spin 2 that the $\mathcal{A}^{(-)}\left[\phi^{(2,0)}\right]$ correspond to massless gravitons with 2 degrees of freedom, while the remaining $\mathcal{A}^{(-)}\left[\phi^{(2,1)}\right]$ and $\mathcal{A}^{(-)}\left[\phi^{(2,2)}\right]$ contribute the remaining 3 degrees of freedom for a generic spin 2 mode.

Quadratic form for $\mathcal{D}^{2}$. Using the above results, we obtain the quadratic form for $\mathcal{D}^{2}$ as follows:

$$
\begin{aligned}
\int \mathcal{A}_{\mu}^{(g)}\left[\phi^{\prime}\right]\left(\mathcal{D}^{2}+\frac{1}{2} \mu^{2}\right) \mathcal{A}^{(g) \mu}[\phi] & =\int \square \phi^{\prime} \square \phi \\
\int \mathcal{A}_{\mu}^{(+)}\left[\phi^{\prime(s)}\right]\left(\mathcal{D}^{2}+\frac{1}{2} \mu^{2}\right) \mathcal{A}^{(g) \mu}\left[\phi^{(s+1)}\right] & =-\frac{s+3}{R} \int D^{+} \phi^{\prime(s)} \square \phi^{(s+1)} \\
\int \mathcal{A}_{\mu}^{(-)}\left[\phi^{\prime(s)}\right]\left(\mathcal{D}^{2}+\frac{1}{2} \mu^{2}\right) \mathcal{A}^{(g) \mu}\left[\phi^{(s-1)}\right] & =-\frac{-s+2}{R} \int D^{-} \phi^{(s)} \square \phi^{(s-1)} \\
\int \mathcal{A}_{\mu}^{(+)}\left[\phi^{\prime(s-1)}\right]\left(\mathcal{D}^{2}+\frac{1}{2} \mu^{2}\right) \mathcal{A}^{(-) \mu}\left[\phi^{(s+1)}\right] & =-\int D^{+} \phi^{\prime(s-1)} D^{-}\left(\square+\frac{-2 s}{R^{2}}\right) \phi^{(s+1)} \\
\int \mathcal{A}_{\mu}^{(\tau)}\left[\phi^{\prime}\right]\left(\mathcal{D}^{2}+\frac{1}{2} \mu^{2}\right) \mathcal{A}^{(4) \mu}[\phi] & =-\int R^{2} \cosh ^{2}(\eta) \phi^{\prime}\left(\square+\frac{4}{R^{2}}\right) \phi \\
& -\int 2 \phi^{\prime} \sinh ^{2}(\eta)(s+\tau) \phi
\end{aligned}
$$

using (2.36), (5.27), (5.58i), (A.35), and (A.38). As a consistency check, we also compute

$$
\begin{aligned}
\int\left(\mathcal{D}^{2}+\frac{1}{2} \mu^{2}\right) \mathcal{A}_{\mu}^{(+)}\left[\phi^{(s)}\right] \mathcal{A}^{(g) \mu}\left[\phi^{(s+1)}\right] & =-\frac{s+3}{R} \int \square D^{+} \phi^{(s)} \phi^{(s+1)}, \\
\int\left(\mathcal{D}^{2}+\frac{1}{2} \mu^{2}\right) \mathcal{A}_{\mu}^{(-)}\left[\phi^{\prime(s)}\right] \mathcal{A}^{(g) \mu}\left[\phi^{(s-1)}\right] & =-\frac{-s+2}{R} \int D^{-} \phi^{(s)} \square \phi^{(s-1)}, \\
\int\left(\mathcal{D}^{2}+\frac{1}{2} \mu^{2}\right) \mathcal{A}_{\mu}^{(+)}\left[\phi^{\prime}\right] \mathcal{A}^{(-) \mu}[\phi] & =-\int \square D^{+} \phi^{\prime} D^{-} \phi .
\end{aligned}
$$

in agreement with the above. The terms $\int \mathcal{A}_{\mu}^{(\tau)}\left(\mathcal{D}^{2}+\frac{1}{2} \mu^{2}\right) \mathcal{A}^{(i) \mu}$ for $i \in\{ \pm, g\}$ can be evaluated using the above results for $\mathcal{D}^{2} \mathcal{A}^{(i)}$, and we skip the verification of Hermiticity for $\mathcal{D}^{2}$. As expected, the kinetic term for time-like $\mathcal{A}_{\mu}^{(\tau)}$ has a negative sign, but this mode is not part of the physical Hilbert space. Nevertheless, these modes are needed to compute the propagator.

\section{Metric and gravitons on $\mathcal{M}^{3,1}$}

In this paper, we consider only the basic metric aspects of linearized gravity. Further developments and more formal aspects will be studied elsewhere. The effective metric on a perturbed background is extracted from the kinetic term as in (2.60), and is formally obtained from the bi-derivation

$$
\begin{aligned}
\gamma: \mathcal{C} \times \mathcal{C} & \rightarrow \mathcal{C} \\
\left(\phi, \phi^{\prime}\right) & \mapsto\left\{Y^{\alpha}, \phi\right\}\left\{Y_{\alpha}, \phi^{\prime}\right\}
\end{aligned}
$$


up to a conformal factor as discussed in section 2.5. Specializing to $\phi=x^{\mu}, \phi^{\prime}=x^{\nu}$ we obtain the form $\gamma^{\mu \nu}=\bar{\gamma}^{\mu \nu}+\delta_{\mathcal{A}} \gamma^{\mu \nu}+O\left(\mathcal{A}^{2}\right)$ in Cartesian coordinates, with metric fluctuation given by

$$
\begin{aligned}
\delta_{\mathcal{A}} \gamma^{\mu \nu} & :=\left\{t^{\alpha}, x^{\mu}\right\}\left\{\mathcal{A}_{\alpha}, x^{\nu}\right\}+(\mu \leftrightarrow \nu) \\
& =\sinh (\eta)\left\{\mathcal{A}_{\mu}, x^{\nu}\right\}+(\mu \leftrightarrow \nu) .
\end{aligned}
$$

To evaluate this for the above $\mathcal{A}^{(i)}$ modes, it is convenient to consider the following rescaled graviton mode:

$$
\begin{aligned}
H^{\mu \nu}[\mathcal{A}] & :=\frac{1}{\sinh (\eta)} \delta_{\mathcal{A}} \gamma^{\mu \nu}=\left\{\mathcal{A}^{\mu}, x^{\nu}\right\}+(\mu \leftrightarrow \nu) \\
h^{\mu \nu}[\mathcal{A}] & :=\left[H^{\mu \nu}[\mathcal{A}]\right]_{0}=\left\{\mathcal{A}^{\mu}, x^{\nu}\right\}_{-}+(\mu \leftrightarrow \nu) .
\end{aligned}
$$

Clearly only $\mathcal{A} \in \mathcal{C}^{1}$ can contribute to $h^{\mu \nu}[\mathcal{A}]$. The trace contributions are

$$
H[\mathcal{A}]=2\left\{\mathcal{A}^{\mu}, x_{\nu}\right\}, \quad h[\mathcal{A}]=2\left\{\mathcal{A}^{\mu}, x_{\mu}\right\}_{0} .
$$

We observe

$$
\begin{aligned}
\left\{t_{\mu}, h^{\mu \nu}[\mathcal{A}]\right\} & =\left\{\left\{t_{\mu}, \mathcal{A}^{\mu}\right\}, x^{\nu}\right\}_{-}-\frac{2}{R} D^{-} \mathcal{A}^{\nu} \\
& =-\frac{2}{R} D^{-} \mathcal{A}^{\nu}
\end{aligned}
$$

using (A.35) for $\mathcal{A}^{\nu} \in \mathcal{C}^{1}$, and assuming the gauge-fixing $\left\{t_{\mu}, \mathcal{A}^{\mu}\right\}=0$ in the last line. Then

$$
\begin{aligned}
\left\{t_{\nu},\left\{t_{\mu}, h^{\mu \nu}[\mathcal{A}]\right\}\right\} & =-\left\{t_{\nu},\left\{x^{\nu},\left\{t_{\mu}, \mathcal{A}^{\mu}\right\}\right\}_{-}\right\}-\frac{2}{R}\left\{t_{\nu}, D^{-} \mathcal{A}^{\nu}\right\} \\
& =-\frac{1}{R} D^{-}\left\{t_{\mu}, \mathcal{A}^{\mu}\right\}-\frac{2}{R}\left\{t_{\nu}, D^{-} \mathcal{A}^{\nu}\right\} \\
& =-\frac{3}{R} D^{-}\left\{t_{\mu}, \mathcal{A}^{\mu}\right\}-\frac{1}{R^{2}} h
\end{aligned}
$$

However, recall that the effective metric, as identified in section 2.5, differs from the above by a conformal factor: hence, (2.62) becomes

$$
G^{\mu \nu}=\bar{G}^{\mu \nu}+\delta G^{\mu \nu}, \quad \text { with } \quad \delta G^{\mu \nu}:=\alpha\left[\delta_{\mathcal{A}} \gamma^{\mu \nu}-\frac{1}{2} \eta^{\mu \nu}\left(\eta_{\alpha \beta} \delta_{\mathcal{A}} \gamma^{\alpha \beta}\right)\right]_{0},
$$

where $\bar{G}^{\mu \nu}=\alpha \gamma^{\mu \nu}=\sinh ^{-1}(\eta) \eta^{\mu \nu}$, see (2.63), is the effective background metric, and $\alpha=\sinh ^{-3}(\eta)$ is the conformal factor arising from the fixed symplectic measure on $\mathbb{C} P^{1,2}$. Note that $\eta^{\mu \nu} \eta_{\alpha \beta}=\gamma^{\mu \nu} \gamma_{\alpha \beta}$, i.e. the conformal factor drops out here. We therefore proceed with the auxiliary metric fluctuation $h^{\mu \nu}$. 
Pure gauge modes. Suppose $\phi=\phi^{(1)}$ is a spin 1 field. Then the auxiliary metric fluctuation of $\mathcal{A}^{(g)}$ has the following properties:

$$
\begin{aligned}
h_{(g)}^{\mu \nu}[\phi] & :=h^{\mu \nu}\left[\mathcal{A}^{(g)}\right]=-\left\{t^{\mu}, \mathcal{A}^{\nu(-)}[\phi]\right\}+(\mu \leftrightarrow \nu)+\frac{2}{R} D^{-} \phi \eta^{\mu \nu} \\
& =-\left\{t^{\mu}, \mathcal{A}^{\nu(-)}[\phi]\right\}+(\mu \leftrightarrow \nu)+\frac{1}{3} h^{(g)} \eta^{\mu \nu}, \\
h^{(g)}[\phi] & :=h\left[\mathcal{A}^{(g)}\right]=-2\left\{t_{\mu}, \mathcal{A}^{\mu(-)}\left[\phi^{(1)}\right]\right\}+\frac{8}{R} D^{-} \phi=\frac{6}{R} D^{-} \phi \\
& =6\left\{t_{\mu}, \mathcal{A}^{\mu(-)}\left[\phi^{(1)}\right]\right\} \\
\left\{t_{\mu}, h^{\mu \nu}\left[\mathcal{A}^{(g)}\right]\right\} & =-\left\{\square \phi, x^{\nu}\right\}_{-}-\frac{2}{R} D^{-}\left\{t^{\nu}, \phi\right\}, \\
\left\{t_{\mu},\left\{t^{\alpha}, h_{\alpha \nu}^{(g)}\right\}\right\} & =-\frac{1}{3}\left\{t_{\mu},\left\{t_{\nu}, h^{(g)}\right\}\right\}+\left\{t_{\mu},\left\{x_{\nu},\left(\square-\frac{2}{R^{2}}\right) \phi\right\}_{-}\right\},
\end{aligned}
$$

using (A.35). Hence the $h_{(g)}^{\mu \nu}$ are traceless for divergence-free modes $\phi^{(1,0)}$. Even for the $\phi^{(1,1)}=D \phi^{(0)}$ modes, the trace contribution $h$ is subleading and can be dropped at scales much shorter than the cosmological scales. To see this, consider for $\phi=\phi^{(0)}$

$$
\begin{aligned}
h_{\mu \nu}^{(g)}[D \phi] & =-\left\{x_{\mu},\left\{t_{\nu}, D \phi\right\}\right\}+(\mu \leftrightarrow \nu) \\
& =O\left(r^{2} R^{2} \cosh ^{3}(\eta)\right) \partial \partial \partial \phi+(\mu \leftrightarrow \nu) \\
h^{(g)}[D \phi] & =\frac{6}{R} D^{-} D \phi=O\left(r^{2} R \cosh ^{2}(\eta)\right) \Delta_{3} \phi
\end{aligned}
$$

noting that $D(\phi)=O(\theta \partial \phi)$ due to (3.32). Therefore

$$
h_{\mu \nu}^{(g)}=O(x \partial) h^{(g)} \gg h^{(g)} .
$$

Therefore the pure gauge modes are effectively traceless for sufficiently short scales, and $h_{\mu \nu}^{(g)}$ has the usual form $\sim \partial_{\mu} \mathcal{A}_{\nu}+\partial_{\nu} \mathcal{A}_{\mu}$ of volume-preserving diffeomorphisms due to (6.8a). Finally, we note that the gauge transformation of the combination

$$
\delta_{\phi}\left(h_{\mu \nu}-\frac{1}{3} \eta_{\mu \nu} h\right)=h_{\mu \nu}^{(g)}-\frac{1}{3} \eta_{\mu \nu} h^{(g)}=-\left\{t^{\mu}, \mathcal{A}^{\nu(-)}[\phi]\right\}+(\mu \leftrightarrow \nu)
$$

is close to the usual form of a pure gauge diffeomorphism contribution, determined by $\phi \in \mathcal{C}^{(1)}$. This will be a useful starting point for the construction of a linearized EinsteinHilbert-like action. In particular, since the trace-contribution is subleading, the different factors $\frac{1}{3}$ versus $\frac{1}{2}$ in (6.7) is not significant.

Physical $\mathcal{A}^{(-)}$modes. Among the $\mathcal{A}^{(-)}\left[\phi^{(s)}\right]$ modes, only the ones with spin $s=2$ can contribute to the metric, as

$$
\begin{aligned}
h_{(-)}^{\mu \nu}\left[\phi^{(2)}\right] & :=h^{\mu \nu}\left[\mathcal{A}^{(-)}\left[\phi^{(2)}\right]\right]=-\left\{x^{\mu},\left\{x^{\nu}, \phi^{(2)}\right\}_{-}\right\}_{-}+(\mu \leftrightarrow \nu), \\
h_{(-)} & :=\eta_{\mu \nu} h_{(-)}^{\mu \nu}=-2\left\{x^{\mu},\left\{x_{\mu}, \phi^{(2)}\right\}_{-}\right\}_{-}=2 D_{-} D_{-} \phi^{(2)}, \\
\left\{t_{\mu}, h_{(-)}^{\mu \nu}\left[\phi^{(2)}\right]\right\} & =-\frac{2}{R}\left\{x^{\nu}, D^{-} \phi^{(2)}\right\}_{-}=-\frac{2}{R} \mathcal{A}_{\nu}^{(-)}\left[D^{-} \phi^{(2)}\right], \\
\left\{t_{\mu},\left\{t^{\alpha}, h_{\alpha \nu}^{(-)}\right\}\right\}+(\mu \leftrightarrow \nu) & =\frac{2}{R^{2}}\left(h_{\mu \nu}^{(g)}-\frac{1}{3} \eta_{\mu \nu} h^{(g)}\right)\left[D^{-} \phi^{(2)}\right]
\end{aligned}
$$


using (3.49) and (6.5), cf. (3.43). Recall from (5.42) that $\mathcal{A}^{(-)}\left[\phi^{(2)}\right]$ is automatically gaugefixed. Therefore the physical Hilbert space $\mathcal{H}_{\text {phys }}$ contains not only $\mathcal{A}^{(-)}\left[\phi^{(2,0)}\right]$, but also $\mathcal{A}^{(-)}\left[\phi^{(2,1)}\right]$ and $\mathcal{A}^{(-)}\left[\phi^{(2,2)}\right]$, unless the last two are equivalent to pure gauge modes. Moreover, (6.12b) shows that $h_{(-)}$vanishes for $\phi^{(2,0)}$ and $\phi^{(2,1)}$, but it is non-vanishing for $\phi^{(2,2)}$. The $h_{\mu \nu}^{(-)}\left[\phi^{(2,0)}\right]$ are divergence-free and traceless gravitons, which realize the usual 2 propagating physical degrees of freedom of GR. The $h_{\mu \nu}^{(-)}\left[\phi^{(2,1)}\right]$ lead to graviton modes which turn out to be Ricci-flat on-shell in section 6.2 , and therefore may be equivalent ${ }^{24}$ to unphysical pure gauge modes. Finally, $h_{\mu \nu}^{(-)}\left[D^{+} D^{+} \phi^{(0)}\right]$ provides an extra propagating scalar metric mode which has no counterpart in GR; its significance should be clarified elsewhere. Off-shell, the modes in $h_{\mu \nu}^{(-)}\left[\phi^{(2)}\right]$ provide the degrees of freedom of a massive spin 2 multiplet. These modes are approximately in de Donder gauge, since (6.12c) is suppressed at scales shorter than the cosmological curvature scale.

Unphysical $\mathcal{A}^{(+)}$modes. None of the $\mathcal{A}^{(+)}\left[\phi^{(s)}\right]$ modes with $s \geq 1$ can contribute to $h^{\mu \nu}$, because

$$
H^{\mu \nu}\left[\mathcal{A}^{(+)}\left[\phi^{(s)}\right]\right]=-\left\{x^{\mu},\left\{x^{\nu}, \phi^{(s)}\right\}_{+}\right\}+(\mu \leftrightarrow \nu) \quad \in \mathcal{C}^{s} \oplus \mathcal{C}^{s+2} .
$$

However the scalar $\mathcal{A}^{(+)}\left[\phi^{(0)}\right]$ mode contributes, which gives

$$
\begin{aligned}
h_{(+)}^{\mu \nu}\left[\phi^{(0)}\right]= & -\left\{x^{\mu},\left\{x^{\nu}, \phi^{(0)}\right\}_{+}\right\}_{-}+(\mu \leftrightarrow \nu) \\
= & -2\left[\theta^{\mu \alpha} \theta^{\nu \beta}\right]_{0} \partial_{\alpha} \partial_{\beta} \phi-\left(\left\{x^{\mu}, \theta^{\nu \beta}\right\} \partial_{\beta}+\left\{x^{\nu}, \theta^{\mu \beta}\right\} \partial_{\beta}\right) \phi^{(0)} \\
= & -\frac{2 r^{2} R^{2}}{3}\left(P^{\mu \nu} P^{\alpha \beta}-P^{\mu \beta} P^{\nu \alpha}\right) \partial_{\alpha} \partial_{\beta} \phi^{(0)}-\left(\left\{x^{\mu}, \theta^{\nu \beta}\right\} \partial_{\beta}+\left\{x^{\nu}, \theta^{\mu \beta}\right\} \partial_{\beta}\right) \phi^{(0)} \\
= & \frac{2 r^{2} R^{2}}{3}\left(\partial^{\mu} \partial^{\nu}-\left(\eta^{\mu \nu}+R^{-2} x^{\mu} x^{\nu}\right) \partial^{\alpha} \partial_{\alpha}\right) \phi^{(0)} \\
& -\frac{2}{3} r^{2} \eta^{\mu \nu}(\tau+2) \tau \phi^{(0)}+\frac{1}{3} r^{2}\left(x^{\nu} \partial^{\mu}+x^{\mu} \partial^{\nu}\right)(1+2 \tau) \phi^{(0)} \\
h_{(+)}= & -2\left\{x_{\mu},\left\{x^{\mu}, \phi^{(0)}\right\}\right\}_{-}=-2\left\{x_{\mu},\left\{x^{\mu}, \phi^{(0)}\right\}\right\}_{0} \\
\left\{t_{\mu}, h_{(+)}^{\mu \nu}\right\}= & \left\{\left\{t_{\mu}, \mathcal{A}^{(+) \mu}\right\}, x^{\nu}\right\}_{-}-\frac{2}{R} D^{-} \mathcal{A}^{(+) \nu} \\
= & \frac{3}{R}\left\{D^{+} \phi^{(0)}, x^{\nu}\right\}_{-}-\frac{2}{R} D^{-} \mathcal{A}^{(+) \nu}=-2 r^{2}\left\{t^{\nu}, \phi^{(0)}\right\}-\frac{5}{R}\left\{x^{\nu}, D \phi^{(0)}\right\}_{-}
\end{aligned}
$$

recalling $\tau=x \partial$ and using (2.55), (5.42), and (6.5). Even though $\mathcal{A}^{(+)}\left[\phi^{(0)}\right]$ is unphysical, this provides the missing scalar degree of freedom for the off-shell metric fluctuations. Finally, $\mathcal{A}^{(\tau)}$ is not expected to provide an additional physical mode, and will not be considered any further here.

Discussion. To summarize, we have found 9 independent off-shell metric fluctuations: 5 from the $\mathcal{A}^{(-)}\left[\phi^{(2)}\right]$ modes, one from the scalar $\mathcal{A}^{(+)}\left[\phi^{(0)}\right]$ mode, and the 3 pure gauge modes. All these modes are governed by the appropriate wave equations (5.55), which are massless up to cosmological scales. There may be an extra off-shell (unphysical) mode based

\footnotetext{
${ }^{24}$ in the present approximation which is restricted to scales shorter than the cosmic curvature scale, we cannot decide whether the $h_{\mu \nu}^{(-)}\left[\phi^{(2,1)}\right]$ are exactly or only approximately equal to pure gauge modes.
} 
on $\mathcal{A}^{(\tau)}$, which should be clarified elsewhere. These metric fluctuations provide all $(5+1)$ degrees of freedom required for gravity in the presence of matter. However, in contrast to GR there are only 3 instead of 4 pure gauge modes $\mathcal{A}^{(g)}$, corresponding essentially to volume-preserving diffeomorphisms ${ }^{25}(6.10)$. We expect that this is also related to the absence of a cosmological constant term as discussed in section 6.3.

On-shell, the physical Hilbert space $\mathcal{H}_{\text {phys }}$ of vacuum modes certainly contains the 2 standard Ricci-flat graviton modes, while the significance of the other modes remains to be understood.

These gravitational modes will certainly be sourced by matter. However to obtain the linearized Einstein equations to a sufficiently good approximation may require an induced Einstein-Hilbert-like action, as discussed below. In fact, induced gravity actions are known to arise quite generically upon taking quantum effects into account [46, 68]. The large gauge invariance of the present framework will allow to determine these actions, as shown in the remaining sections.

\subsection{Linearized curvature-like tensors}

To understand the significance of the above metric modes, we consider their linearized Ricci tensor. Recall that the linearized Ricci tensor $\mathcal{R}_{(\text {lin) }}^{\mu \nu}$ for a metric fluctuation $\delta g_{\mu \nu}$ is given by

$$
\begin{aligned}
R_{(\text {lin })}[\delta g]= & -\nabla^{\alpha} \nabla_{\alpha} \delta g^{\mu \nu}+\nabla^{\mu} \nabla_{\rho} \delta g^{\nu \rho}+\nabla^{\nu} \nabla_{\rho} \delta g^{\mu \rho}-\nabla_{\mu} \nabla_{\nu} \delta g \\
& +\eta^{\mu \nu}\left(\nabla^{\alpha} \nabla_{\alpha} \delta g-\nabla_{\sigma} \nabla_{\rho} \delta g^{\sigma \rho}\right) .
\end{aligned}
$$

We need to find analogous expressions that are suitable for the matrix model setting, which should be defined solely in terms of (commutators or) Poisson brackets. We tentatively define a linearized Poisson-Ricci tensor $\mathcal{R}_{(\mathrm{lin})}^{\mu \nu}$ and scalar $\mathcal{R}_{(\mathrm{lin})}$, as well as a linearized Poisson-Einstein tensor $\mathcal{G}_{(\mathrm{lin})}^{\mu \nu}$ as follows:

$$
\begin{aligned}
2 \mathcal{R}_{(\text {lin })}^{\mu \nu}[\delta g]= & \square \delta g^{\mu \nu}+\left(\left\{t_{\mu},\left\{t_{\rho}, \delta g^{\rho \nu}\right\}\right\}-\frac{1}{2}\left\{t_{\mu},\left\{t_{\nu}, \delta g\right\}\right\}+(\mu \leftrightarrow \nu)\right), \\
\mathcal{R}_{(\text {lin })}= & \square \delta g+\left\{t_{\mu},\left\{t_{\rho}, \delta g^{\rho \mu}\right\}\right\}, \\
2 \mathcal{G}_{(\text {lin })}^{\mu \nu}[\delta g]= & 2 R_{(\text {lin })}^{\mu \nu}[\delta g]-\eta^{\mu \nu} R_{(\text {lin })}[\delta g] \\
= & \square \delta g^{\mu \nu}+\left(\left\{t_{\mu},\left\{t_{\rho}, \delta g^{\rho \nu}\right\}\right\}-\frac{1}{2}\left\{t_{\mu},\left\{t_{\nu}, \delta g\right\}\right\}+(\mu \leftrightarrow \nu)\right) \\
& -\eta^{\mu \nu}\left(\square \delta g+\left\{t_{\sigma},\left\{t_{\rho}, \delta g^{\sigma \rho}\right\}\right\}\right), \\
\mathcal{G}_{(\text {lin })}= & -\left(\square \delta g+\left\{t_{\mu},\left\{t_{\rho}, \delta g^{\rho \mu}\right\}\right\}\right) .
\end{aligned}
$$

To understand these expressions in terms of usual tensor calculus, we recall $\left\{t_{\mu}, \cdot\right\} \sim$ $\sinh (\eta) \partial_{\mu}$ in Cartesian (background) coordinates, and similarly $\square \sim \sinh ^{2}(\eta)\left(\eta^{\mu \nu} \partial_{\mu} \partial_{\nu}+\right.$ $\left.O\left(\frac{1}{x_{4}} \partial\right)\right)$ from $(2.75)$, because

$$
\frac{1}{\sinh (\eta)} \partial \sinh (\eta)=O\left(\frac{1}{x_{4}}\right)
$$

\footnotetext{
${ }^{25}$ They preserve the invariant symplectic volume form on $\mathbb{C} P^{1,2}$.
} 
and $x_{4}=R \sinh (\eta)$ measures the current size of the universe. Then the linearized PoissonRicci tensor (6.16) reduces to

$$
\begin{aligned}
\mathcal{R}_{(\text {lin })}^{\mu \nu}\left[\delta g^{\alpha \beta}\right] & =\frac{1}{2} \square \delta g^{\mu \nu}+\left(\left\{t^{\mu},\left\{t_{\alpha}, \delta g^{\alpha \nu}\right\}\right\}-\frac{1}{2}\left\{t^{\mu},\left\{t^{\nu}, \delta g\right\}\right\}+(\mu \leftrightarrow \nu)\right) \\
& =\frac{1}{2} \sinh ^{2}(\eta)\left(-\partial^{\alpha} \partial_{\alpha} \delta g^{\mu \nu}-\partial^{\mu} \partial^{\mu} \delta g+\partial^{\mu} \partial_{\rho} \delta g^{\nu \rho}+\partial^{\nu} \partial_{\rho} \delta g^{\mu \rho}+O\left(\frac{1}{x_{4}} \partial \delta g\right)\right) \\
& =\sinh ^{2}(\eta)\left(R_{(\operatorname{lin})}^{\mu \nu}\left[\delta g^{\alpha \beta}\right]+O\left(\frac{1}{x_{4}} \partial \delta g\right)\right) .
\end{aligned}
$$

Up to the sub-leading corrections, this is the classical linearized Ricci tensor for a metric fluctuation $\delta g^{\mu \nu}$ around a background $\bar{G}^{\mu \nu}=\sinh ^{2}(\bar{\eta}) \eta^{\mu \nu}$, or some suitable conformal rescaling thereof such as (2.63). Note that the indices in the second line are contracted with $\eta_{a \beta}$.

\subsection{Curvature and gravitational waves}

Now we relate the linearized Ricci tensor to the equations of motion of the physical modes $\mathcal{A}^{( \pm)}$underlying the metric fluctuations, and show that the linearized gravitational waves of GR are recovered.

To evaluate the Ricci tensor, we need a relation for $\square h^{\mu \nu}$. This can be achieved using the intertwiner relation (A.49),

$$
\mathcal{D}^{2} h^{\mu \nu}[\mathcal{A}]=\left(\square+\frac{2}{R^{2} r^{2}} \tilde{\mathcal{I}}\right) h^{\mu \nu}[\mathcal{A}]=h_{\mu \nu}\left[\mathcal{D}^{2} \mathcal{A}\right]+\frac{2}{R^{2}}\left(3 h^{\mu \nu}[\mathcal{A}]-\eta^{\mu \nu} h[\mathcal{A}]\right)
$$

and noting that $\mathcal{D}^{2}=\square+O\left(\frac{1}{x_{4}} \partial\right) \approx \square$ up to corrections of the order of the cosmological curvature. Let us discuss this for the different modes.

Pure gauge modes. Consider first the pure gauge mode $\mathcal{A}^{(g)}[\phi]$. From the properties derived in (6.8) we obtain for the Poisson-Ricci tensor

$$
\begin{aligned}
2 \mathcal{R}_{(\operatorname{lin})}^{\mu \nu}\left[h^{(g)}[\phi]\right]= & \square h_{\mu \nu}^{(g)}+\frac{2}{R^{2}}\left(h_{\mu \nu}^{(g)}-\frac{1}{3} \eta_{\mu \nu} h^{(g)}\right)[\phi]-\left(h_{\mu \nu}^{(g)}-\frac{1}{3} \eta_{\mu \nu} h^{(g)}\right)[\square \phi] \\
& -\left(\frac{1}{2}+\frac{1}{3}\right)\left(\left\{t_{\mu},\left\{t_{\nu}, h^{(g)}\right\}\right\}+(\mu \leftrightarrow \nu)\right) \\
\approx & \square h_{\mu \nu}^{(g)}-h_{\mu \nu}^{(g)}[\square \phi]
\end{aligned}
$$

at sufficiently short scales, using (6.10) and dropping $\frac{1}{R^{2}}$ terms. Together with (6.20) to this approximation, ${ }^{26}$ the linearized Ricci tensor (6.21) vanishes both on and off shell as it should,

$$
R_{(\operatorname{lin})}^{\mu \nu}[h]=0+O\left(\frac{1}{x_{4}} \partial h\right) .
$$

\footnotetext{
${ }^{26}$ Of course these modes cannot have any physical effect whatsoever. This is just a consistency check for the approximation which is used for the other, physical modes.
} 
Physical $\mathcal{A}^{(-)}$modes. Now consider $\mathcal{A}^{(-)}\left[\phi^{(2)}\right]$, for which the properties listed in (6.12) imply

$$
\begin{aligned}
2 \mathcal{R}_{(\text {lin })}^{\mu \nu}\left[h^{(-)}[\phi]\right] & =\square h_{\mu \nu}^{(-)}+\frac{2}{R^{2}}\left(h_{\mu \nu}^{(g)}-\frac{1}{3} \eta_{\mu \nu} h^{(g)}\right)\left[D^{-} \phi\right]+\left(\left\{t_{\mu},\left\{t_{\nu}, D^{-} D^{-} \phi\right\}\right\}+(\mu \leftrightarrow \nu)\right) \\
& \approx \square h_{\mu \nu}^{(-)}+\left(\left\{t_{\mu},\left\{t_{\nu}, D^{-} D^{-} \phi\right\}\right\}+(\mu \leftrightarrow \nu)\right) \\
& =\square h_{\mu \nu}^{(-)}+\frac{1}{2}\left(\left\{t_{\mu},\left\{t_{\nu}, h^{(-)}\right\}\right\}+(\mu \leftrightarrow \nu)\right)
\end{aligned}
$$

dropping $\frac{1}{R^{2}}$ terms. We will see that this vanishes on-shell but not off-shell for $\mathcal{A}^{(-)}\left[\phi^{(2,0)}\right]$ and $\mathcal{A}^{(-)}\left[\phi^{(2,1)}\right]$.

Consider first the 2 physical modes $\mathcal{A}^{(-)}\left[\phi^{(2,0)}\right]$. The on-shell condition (5.55) for the bare action is $\left(\square-\frac{4}{R^{2}}\right) \phi^{(2,0)}=0$, and the corresponding metric fluctuation $h_{\mu \nu}$ is traceless and satisfies $\left\{t^{\mu}, h_{\mu \nu}\right\}=0$ due to (6.12c). It follows with (6.20) that the linearized Ricci tensor vanishes on-shell up to corrections of the order of the cosmological curvature,

$$
\sinh ^{2}(\eta) R_{(\text {lin })}^{\mu \nu}[h] \approx \mathcal{R}_{(\text {lin })}^{\mu \nu}[h] \approx \frac{1}{2} h^{\mu \nu}\left[\mathcal{D}^{2} \mathcal{A}^{(-)}\right]=0+O\left(\frac{1}{x_{4}} \partial h\right) .
$$

These are essentially the standard Ricci-flat gravitational wave solutions of GR, which are thus recovered in the bare matrix model. This is one of the main results of this paper. In the presence of an induced Einstein-Hilbert term $S_{\mathrm{EH}}$ (6.34) as discussed below, these will of course remain to be solutions, up to small corrections.

Now consider the physical modes $\mathcal{A}^{(-)}\left[\phi^{(2,1)}\right]$. Since they are trace-free $(6.12 \mathrm{~b})$, it follows with (6.20) that they are also Ricci-flat up to subleading corrections,

$$
R_{(\operatorname{lin})}^{\mu \nu}[h]=0+O\left(\frac{1}{x_{4}} \partial h\right) .
$$

In general, there exist only 2 independent Ricci-flat metric modes (apart from trivial diffeomorphism modes). Therefore in this approximation, the $\mathcal{A}^{(-)}\left[\phi^{(2,1)}\right]$ should not be independent modes. This would suggest that the present theory contains only the 2 propagating metric modes of massless spin 2 gravitons as in GR, rather than the 5 propagating modes of massive gravitons. However, is conceivable that the latter are masked by pure gauge modes, and are not visible in the present approximation. To settle this question, a more refined analysis is required.

Now consider the scalar metric mode arising from $\mathcal{A}^{(-)}\left[\phi^{(2,2)}\right]$. This is not expected to be Ricci-flat in general, and provides an extra physical mode which has no counterpart in GR. This is not surprising due to the reduced gauge invariance, corresponding to a 3-parameter volume-preserving diffeomorphism group which preserves the invariant volume form. The existence of extra scalar modes in modified theories of gravity is not uncommon and may be of interest e.g. in the context of cosmology or possibly dark matter. However, we leave a more detailed investigation to future work. Finally, the $\mathcal{A}^{(+)}\left[\phi^{(0)}\right]$ mode is not physical and hence discarded.

In the presence of an induced Einstein-Hilbert action $S_{\mathrm{EH}}$ (6.34) discussed below, the 2 propagating Ricci-flat modes will clearly survive, while the fate of the scalar gravity mode 
remains to be understood. Of course off-shell, all $5+1$ non-trivial metric modes are present, which are needed to describe gravity in the presence of matter. We therefore expect to recover linearized GR to a good approximation, presumably with an extra scalar mode, and a non-linear completion defined by the matrix model.

\subsection{Linearized Einstein-Hilbert-like action}

In this final section, we derive an action for $h^{\mu \nu}$ which plays the role of the linearized Einstein-Hilbert (E-H) action. The strategy for the construction is based on two requirements: first, the action should reduce to the usual linearized E-H action in the appropriate limit and, second, the action should be gauge invariant. We obtain such an action which is expressed solely in terms of Poisson brackets, as appropriate for the present framework. Much of the following considerations would generalize to higher-spin modes. However to keeps things within bounds we restrict ourselves to the spin 2 sector, postponing the general case.

In the classical case, gauge invariance of the Einstein-Hilbert action is guaranteed by the Bianchi identity $\nabla_{\mu} \mathcal{G}^{\mu \nu}=0$. To find a gauge-invariant action, we recall from (6.11) that the combination $h_{\mu \nu}-\frac{1}{3} h \eta_{\mu \nu}$ has a particularly simple gauge transformation. Therefore, consider the action

$$
S_{1}=\int\left(h_{\mu \nu}-\frac{1}{3} h \eta_{\mu \nu}\right) \mathcal{G}_{(\operatorname{lin})}^{\mu \nu}\left[h_{\alpha \beta}-\frac{1}{3} h \eta_{\alpha \beta}\right]
$$

The gauge transformation of the metric fluctuation is given by $\delta_{\phi} h^{\mu \nu}=h_{(g)}^{\mu \nu}[\phi]$ in (6.8a), which is non-vanishing only for spin 1 gauge transformations generated by $\phi \in \mathcal{C}^{1}$. Since the bracket structure of $\mathcal{G}_{(\text {lin })}^{\mu \nu}(6.17)$ amounts to a self-adjoint operator, the gauge variation of this action is given by

$$
\begin{aligned}
\delta_{\phi} S_{1} & =2 \int \delta_{\phi}\left(h_{\mu \nu}-\frac{1}{3} h \eta_{\mu \nu}\right) \mathcal{G}_{(\text {lin })}^{\mu \nu}\left[h_{\alpha \beta}-\frac{1}{3} h \eta_{\alpha \beta}\right] \\
& =-4 \int\left\{t_{\mu}, \mathcal{A}_{\nu}^{(-)}[\phi]\right\} \mathcal{G}_{(\text {lin })}^{\mu \nu}\left[h_{\alpha \beta}-\frac{1}{3} h \eta_{\alpha \beta}\right] \\
& =4 \int \mathcal{A}_{\nu}^{(-)}[\phi]\left\{t_{\mu}, \mathcal{G}_{(\text {lin })}^{\mu \nu}\left[h_{\alpha \beta}-\frac{1}{3} h \eta_{\alpha \beta}\right]\right\}
\end{aligned}
$$

using (6.8a). Although $\left\{t_{\mu}, \mathcal{G}_{(\text {lin) }}^{\mu \nu}\right\}$ is reminiscent of the Bianchi identity, it does not vanish here, but reduces to (A.17). This will determine the required modifications to obtain a gauge-invariant action. To simplify the evaluation, we define the traceless metric fluctuation

$$
\delta g_{0}^{\mu \nu}=h^{\mu \nu}-\frac{1}{4} \eta^{\mu \nu} h, \quad \delta g_{0}=0 .
$$

In order to compensate the gauge variation of $S_{1}$, we introduce the following quadratic actions:

$$
\begin{aligned}
& S_{h}=\int h \square h, \\
& S_{3}=\int \delta g_{\mu \nu}^{0} \delta g_{0}^{\mu \nu},
\end{aligned}
$$




$$
\begin{aligned}
S_{4} & =\int \delta g_{\mu \nu}^{0}\left\{\mathcal{M}^{\mu \rho}, \delta g_{0}^{\rho \nu}\right\}=\frac{1}{2 r^{2}} \int \delta g_{\mu \nu}^{0} \tilde{\mathcal{I}}\left(\delta g_{0}^{\rho \nu}\right), \\
S_{M 3} & =\int f_{\mu \nu}\left\{\mathcal{M}^{\nu \rho}, h^{\rho \mu}\right\}=-\int h_{\mu \nu}\left\{\mathcal{M}^{\nu \rho}, f^{\rho \mu}\right\}, \\
S_{g f 2} & =R \int\left\{x_{\mu}, A^{\mu}\right\}_{-} D^{-}\left\{t_{\rho}, A^{\rho}\right\}, \\
S_{g f 3} & =\int\left(D^{-}\left\{t_{\nu}, A^{\nu}\right\}\right) \square^{-1}\left(D^{-}\left\{t_{\nu}, A^{\nu}\right\}\right),
\end{aligned}
$$

assuming that $\square^{-1}$ makes sense. For the additional action terms, we employ some shorthand notation

$$
f_{\mu \nu}:=\left\{x^{\mu}, \mathcal{A}^{\nu}\right\}_{-}-\left\{x^{\nu}, \mathcal{A}^{\mu}\right\}_{-}, \quad F_{\mu \nu}[\mathcal{A}]:=\left\{t_{\mu}, \mathcal{A}_{\nu}\right\}-\left\{t_{\nu}, \mathcal{A}_{\mu}\right\}
$$

which satisfy

$$
F_{\mu \nu}\left[\mathcal{A}^{(-)}[\phi]\right]=f_{\nu \mu}\left[\mathcal{A}^{(g)}[\phi]\right]=\delta_{\phi} f_{\nu \mu}
$$

The gauge variations are computed to be (cf. appendix A.1)

$$
\begin{aligned}
\delta_{\phi} S_{h} & =2 \int h^{(g)} \square h, \\
\delta_{\phi} S_{3} & =2 \int \delta g_{0}^{\mu \nu} h_{\mu \nu}^{(g)}, \\
\delta_{\phi} S_{4} & =2 \int \delta g_{0}^{\mu \nu}\left\{\mathcal{M}^{\nu \rho}, h_{\rho \mu}^{(g)}\right\}, \\
\delta_{\phi} S_{M 3} & =-2 \int\left(\left\{x^{\mu}, A^{\nu}\right\}_{-}\left\{\mathcal{M}^{\nu \rho},\left\{x^{\mu}, A_{(g)}^{\rho}\right\}_{-}\right\}-\left\{x^{\nu}, A^{\mu}\right\}_{-}\left\{\mathcal{M}^{\nu \rho},\left\{x^{\rho}, A_{(g)}^{\mu}\right\}_{-}\right\}\right), \\
\delta_{\phi} S_{g f 2} & =R \int\left\{x_{\mu}, A^{\mu}\right\}_{-} D^{-}\left\{t_{\rho}, A_{(g)}^{\rho}\right\}+\left\{x_{\mu}, A_{(g)}^{\mu}\right\}_{-} D^{-}\left\{t_{\rho}, A^{\rho}\right\}, \\
\delta_{\phi} S_{g f 3} & =2 \int\left(D^{-} \phi\right) D^{-}\left(\left\{t_{\nu}, A^{\nu}\right\}\right),
\end{aligned}
$$

using tracelessness of $\delta g_{0}^{\mu \nu}$. These additional action terms allow to compensate the gauge variance of $S_{1}$ and, as detailed in appendix A.1, one explicitly finds

$$
\delta_{\phi} S_{1}=-\frac{3}{2 R^{2}} \delta S_{M 3}+\frac{6}{R^{2}} \delta_{\phi} S_{g f 2}-\frac{9}{2 R^{2}} \delta_{\phi} S_{g f 3}+\frac{1}{2 R^{2}} \delta_{\phi} S_{4}+\frac{1}{R^{2}} \delta_{\phi} S_{3}+\frac{1}{24} \delta_{\phi} S_{h} .
$$

We have thus found the analog of the linearized Einstein-Hilbert action

$$
S_{\mathrm{EH}}:=S_{1}-\frac{1}{24} S_{h}-\frac{1}{R^{2}} S_{3}-\frac{1}{2 R^{2}} S_{4}+\frac{3}{R^{2}}\left(\frac{1}{2} S_{M 3}-2 S_{g f 2}+\frac{3}{2} S_{g f 3}\right)
$$

which is gauge-invariant,

$$
\delta_{\phi} S_{\mathrm{EH}}=0
$$

It is therefore very plausible that this action is induced by quantum corrections at one loop (and beyond), upon integrating out fields coupled to the background with metric 
fluctuation $h^{\mu \nu}$, in the spirit of Sakharov's induced gravity $[46,68]$ or the Seeley-de Wit expansion [69]. Upon gauge-fixing $\mathcal{G}(\mathcal{A}) \equiv\left\{t_{\mu}, \mathcal{A}^{\mu}\right\}=0$, this action simplifies to

$$
S_{\mathrm{EH}, \mathrm{gf}}=S_{1}-\frac{1}{24} S_{h}-\frac{1}{R^{2}}\left(S_{3}+\frac{1}{2} S_{4}-\frac{3}{2} S_{M 3}\right) .
$$

Although we do not have a formal proof, it seems that $S_{\mathrm{EH}}$ of (6.34) is the only gaugeinvariant term at second order of derivatives of $h_{\mu \nu}$; however, this needs to be studied in more detail elsewhere. In particular, there is apparently no gauge-invariant counterpart of the cosmological constant $\int d^{4} x \sqrt{g} \Lambda$. The natural analog is actually the original YangMills action (4.1), which stabilizes the background. This suggests that the cosmological constant problem may not arise here, because the FLRW background is obtained from the bare Yang-Mills-type matrix model action, without fine-tuning and even without requiring any matter. Adding matter would modify the background at shorter scales in a way which should be similar to GR, provided the above linearized Einstein-Hilbert-like action dominates in the quantum effective action.

To understand and interpret the above expressions in terms of usual tensor calculus, we recall the results and approximations in section 6.1, and in particular (6.18). Then the action term $S_{1}$ of (6.26) clearly reduces to the Einstein-Hilbert action for the linearized metric $h_{\mu \nu}-\frac{1}{3} \eta_{\mu \nu} h$ of (6.11), up to corrections suppressed by the cosmological curvature scale. Moreover, recall that the trace $h$ of the physical spin 2 metric modes can be neglected to leading order. However, the trace contribution $S_{h}$ for the scalar metric fluctuations cannot be neglected, and remains to be understood. The terms $S_{4}, S_{M 3}$ and $S_{3}$ in brackets contain only one or no derivative of the metric, and they are suppressed by the cosmological curvature scale just like the subleading corrections in (6.19). This follows from

$$
\frac{1}{R^{2}}\left\{\mathcal{M}^{\mu \nu^{\prime}}, \delta g^{\alpha \beta}\right\} \sim \frac{1}{R^{2}}\left(x^{\mu} \partial^{\nu}-x^{\nu} \partial^{\mu}\right) \delta g^{\alpha \beta} \ll \mathcal{R}_{(\operatorname{lin})}^{\mu \nu}\left[\delta g^{\alpha \beta}\right]
$$

as long as $\partial \gg \frac{1}{x_{4}}$. Hence, $S_{4}, S_{M 3}$, and $S_{3}$ should be negligible for short and intermediate scales, but they may be significant for very long (cosmological) length scales. The remaining terms $S_{g f 2}$ and $S_{g f 2}$ vanish upon gauge-fixing and are not considered any further.

Therefore $S_{\mathrm{EH}}$ reduces to the linearized E-H action for the physical spin 2 modes on short and intermediate scales, but not for the trace contribution. The significance of this deviation is not obvious. Eventually one should also work out the sub-leading contributions $O\left(\frac{1}{x_{4}} \partial \delta g\right)$, and try to interpret them in terms of the cosmological background geometry. These points are postponed to future work.

It is interesting to observe that gauge-invariance apparently requires the non-local contribution $S_{g f 3}$, see (6.29f). Of course, this term vanishes for gauge-fixed fields, leaving a local action whose leading (2nd derivative) contributions are given by $S_{1}-\frac{1}{24} S_{h}$ in (6.26). Nevertheless, such a non-local term is not unreasonable in a quantum effective action.

\subsection{Discussion}

The precise interplay between the Yang-Mills action (5.3) and the above Einstein-Hilbertlike action $S_{\mathrm{EH}}$ (6.34) is not yet clear. In principle, it should be possible to rewrite the 
quadratic Yang-Mills action $S_{2}[\mathcal{A}](5.3)$ for the gravitational modes in terms of the metric fluctuations $h^{\mu \nu}$, as in [40]. This would lead to a non-local action, ${ }^{27}$ which for $\mathcal{A}^{(-)}$has the structure

$$
S_{2}\left[\mathcal{A}^{(-)}\left[\phi^{(2,0)}\right]\right] \propto-\int h^{\mu \nu}\left[\phi^{(2,0)}\right]\left(\square-\frac{2}{R^{2}}\right)\left(\square_{H}-2 r^{2}\right)^{-1} h_{\mu \nu}\left[\phi^{(2,0)}\right]
$$

but we have not yet found an appealing general form. Note that $\left(\square_{H}-\frac{2}{R^{2}}\right)$ is the positivedefinite Euclidean Laplacian on $H^{4}$, so that the inverse is perfectly well-defined and introduces no zeros or poles. However, we can surely say that the full quadratic action $S_{2}+S_{\mathrm{EH}}$ will admit the usual two Ricci-flat gravitational waves of GR as solutions, since they are solutions of both actions. In contrast, the extra non-Ricci-flat mode arising from the scalar mode in $\mathcal{A}^{(-)}\left[\phi^{(2,0}\right]$ and possibly $\mathcal{A}^{(-)}\left[\phi^{(2,1}\right]$ are presumably not solutions of the full action.

In the presence of matter with standard metric coupling

$$
\delta_{\mathcal{A}} S_{\text {matter }}=-\frac{1}{2} \int d^{4} x \delta G^{\mu \nu}[\mathcal{A}] T_{\mu \nu}
$$

the bare matrix model action (6.38) would not lead to the linearized Einstein equations but rather to equations of the form

$$
\left(\square-\frac{2}{R^{2}}\right) h_{\mu \nu} \sim-\left(\square_{H}-2 r^{2}\right) T_{\mu \nu}
$$

The source term $\square_{H} T_{\mu \nu}$ would lead to a short-range metric perturbation, but a small longdistance contribution might survive due to the constant shift. However in the presence of an induced $S_{\mathrm{EH}}$, we expect that the linearized Einstein equations in the presence of matter are recovered to a good approximation at intermediate scales, provided that induced $S_{\mathrm{EH}}$ dominates. At shorter scales, corrections due to the right-hand-side of (6.40) are expected.

At the non-linear level, it is not entirely clear how to proceed. The problem is that the derivations $\left\{t^{\mu}+\mathcal{A}^{\mu}, \cdot\right\}$ defined by a deformed background no longer respect the spin operator $\mathcal{S}^{2}$. It remains to be seen how this can be handled, possibly by defining a deformed spin operator. However this is just a technical rather than a conceptual problem, and it is clear that the matrix model provides a fully non-linear completion of the linearized gravity discussed in this paper. It is also clear that this will not be identical to GR, and significant differences are bound to arise at cosmological scales. This is manifest by the fact that the cosmological background solution is obtained even without any matter. But this is also the regime where our present understanding of gravity is very limited, which is manifest in the big puzzles around dark energy, dark matter, and the related fine-tuning issues. The gravity theory outlined in this paper clearly has the potential to address these problems, and it remains to be seen where it leads to.

\footnotetext{
${ }^{27}$ This simply reflects the fact that the $\mathcal{A}_{\mu}$ are the fundamental fields here, while the metric fluctuations are derived fields. Observe that in contrast to $H_{n}^{4}$ [40], the two box operators do not cancel here, leading to the vacuum Einstein equations even from the bare action.
} 


\section{Conclusion and outlook}

We briefly summarize some of the most significant points of this paper:

- Fuzzy $\mathcal{M}_{n}^{3,1}$ is defined in terms of the minimal discrete series of unitary representations of $\mathrm{SO}(4,2) \cdot \mathcal{M}_{n}^{3,1}$ respects a global $\mathrm{SO}(3,1)$ isometry, but (local) Lorentz invariance is not manifest. It describes 2 -fold cover of a FLRW space-time, linked by a Big Bounce.

- The space of functions on $\mathcal{M}_{n}^{3,1}$ decomposes into different sectors $\oplus \mathcal{C}^{s}$, each of which contains the degrees of freedom of an irreducible massive spin $s-k$ tensor field. The constraints allow some internal gauge freedom, and they can be represented either as irreducible tangential tensor fields on $H^{4}$, or in space-like gauge eliminating the time-like components.

- Due to the lack of Lorentz invariance, these multiplets decompose further into $\mathcal{C}^{s}=$ $\oplus_{k} \mathcal{C}^{(s, k)} \cdot \mathcal{C}^{(s, k)}=\left(D^{+}\right)^{k} \mathcal{C}^{(s-k, 0)}$. The "lowest" sector $\mathcal{C}^{(s-k, 0)}$ has the degrees of freedom of massless spin $s$ fields.

- The matrix model defines a gauge theory of tangential fluctuation modes on $\mathcal{M}_{n}^{3,1}$, with a higher-spin-type gauge invariance. We argue that it is ghost-free even though there are only 3 rather than 4 diffeomorphism modes, due to the internal space-like gauge and the restricted Lorentz invariance. In particular, the (maximally supersymmetric) IKKT matrix model is expected to define a good quantum theory around this background solution.

- The bosonic on-shell fluctuation modes are classified, and found to respect effectively local Lorentz invariance, corresponding to an effective universal metric. In contrast to the Euclidean case [40] they are propagating and are either exactly massless, or massless up to small corrections.

- The effective metric is not fundamental, but a derived quantity determined be the matrix model background and its fluctuations. In particular, the cosmological background solution is not determined by the Friedmann equations. The on-shell fluctuation modes of the effective metric contain those of massless gravitons, plus a scalar mode. The off-shell metric fluctuations provide all degrees of freedom required for gravity in the presence of matter.

- Gauge invariance allows to determine an effective Einstein-Hilbert-type action $S_{\mathrm{EH}}$, which is expected to be induced upon quantization. It appears that no cosmological constant term can exist. This leads to the scenario where the cosmological background is directly determined by the matrix model quite independently of the matter content, while gravity arises only as an effective theory which describes the fluctuations on the background. Deviations from GR at cosmological scales are expected.

This provides a promising basis for a quantum theory of gravity. However there are many open issues and loose ends, which need to be tied up in following work. One task 
is to find and clarify the $4^{\text {th }}$ off-shell mode, presumably based on $\mathcal{A}^{(\tau)}$. Also, the inner product matrix of the triplet of degenerate modes (5.37) should be diagonalized. This would allow to compute the propagator, and to verify explicitly the no-ghost statement in section 5.5. Furthermore, it is important to determine whether or not the extra degrees of freedom of massive gravitons arising from $\mathcal{A}^{(-)}\left[\phi^{(2)}\right]$ are physical, and to clarify their physical significance.

Since we argued that the Einstein-Hilbert-type action $S_{\mathrm{EH}}$ is induced upon integrating out matter, determining the effective Newton constant requires at least a one-loop computation. This is also required in the computation of quantum corrections to the scale parameter $r$ of the background. Since $H_{n}^{4}$ has a similar structure as $S_{N}^{4}$, one should be able to repeat the 1-loop computation along the lines of $[36,70]$. In particular, the techniques developed in [6] should be very helpful. Eventually, the quantum effective action should also allow to justify the choice of a background solution in the matrix model.

Another task is to compute the sub-leading corrections to the curvature and the linearized Einstein equations. Since we have the full expressions, it should be possible to clarify the geometrical meaning of the linearized Ricci-tensors on the cosmological background. This would then allow to give a meaningful comparison with the standard picture of a cosmological constant, and to address possible relations with apparent dark energy.

Furthermore, the significance of the bare action for the gravitational sector should be clarified, and the full equations for gravity in the presence of matter should be derived and studied. Further topics are finding an analog of the Schwarzschild solution, investigating the deviations from GR in detail, etc.

In any case, having a mechanism for gravity on a (3+1)-dimensional solution of the IKKT matrix model is very significant. For example, this may allow to avoid the issue of a landscape in string theory. That problem arises in the standard approach to string theory, because gravity is assumed to originate from the 10-dimensional bulk gravity, which must thus be compactified to 4 dimensions. In the present mechanism this is no longer required; extra structure for low-energy gauge theory can arise in different ways, see e.g. $[65,66]$ and references therein. Ultimately, one should try to relate these analytic studies with non-perturbative simulations as in [12-15].

Looking forward, a particularly interesting perspective is to elaborate the early universe near the Big Bounce. While the late-time $k=-1$ cosmology is not quite in line with the present concordance model, the resulting picture is very reasonable, given the simplicity of the model and the crudeness of our analysis. Ultimately, the present framework could be powerful enough to address also the deep questions around black holes and the resolution of singularities.

\section{Acknowledgments}

Useful conversations with David Berenstein, Stefan Fredenhagen, Karapet Mkrtchyan, Hikaru Kawai, Jun Nishimura, Jan Rosseel, Mark van Raamsdonk, Peter Schupp, Evgeny Skvortsov, and Asato Tsuchiya are gratefully acknowledged, as well as a related collaboration with Joanna Karczmarek. This work was supported by the Austrian Science Fund 
(FWF) grant P28590. Related support by the Action MP1405 QSPACE from the European Cooperation in Science and Technology (COST) is also acknowledged.

\section{A Mathematical supplements and details}

\section{A.1 Derivation of the Einstein-Hilbert-like action}

Here, we provide the details for the derivation of the Einstein-Hilbert-like action (6.34). To begin with, we split the variation (6.27) of $S_{1}$ according to the traceless perturbation (6.28) and obtain

$$
\delta_{\phi} S_{1}=\underbrace{4 \int \mathcal{A}_{\nu}^{(-)}[\phi]\left\{t_{\mu}, \mathcal{G}_{(\text {lin })}^{\mu \nu}\left[\delta g_{0}^{\alpha \beta}\right]\right\}}_{\equiv \Upsilon_{1}} \underbrace{\}-\frac{1}{3} \int \mathcal{A}_{\nu}^{(-)}[\phi]\left\{t_{\mu}, \mathcal{G}_{(\operatorname{lin})}^{\mu \nu}\left[h \eta_{\alpha \beta}\right]\right\}}_{\equiv \Upsilon_{2}} \equiv \Upsilon_{1}+\Upsilon_{2} .
$$

Next, we note that first term in (A.1) can be simplified using (A.17) and (6.31) as

$$
\begin{aligned}
\Upsilon_{1} & =4 \int \mathcal{A}_{\nu}^{(-)}[\phi]\left\{t_{\mu}, \mathcal{G}_{(\operatorname{lin})}^{\mu \nu}\left[\delta g_{0}\right]\right\} \\
& =2 \int \mathcal{A}_{\nu}^{(-)}[\phi]\left(\frac{1}{r^{2} R^{2}}\left(2\left\{t_{\rho},\left\{\theta^{\rho^{\prime} \rho}, \delta g_{0}^{\rho^{\prime} \nu}\right\}\right\}-\left\{t_{\rho},\left\{\theta^{\mu \nu}, \delta g_{0}^{\rho \mu}\right\}\right\}\right)+\frac{2}{R^{2}}\left\{t_{\rho}, \delta g_{0}^{\rho \nu}\right\}\right) \\
& =-\frac{2}{R^{2}} \int\left\{t_{\rho}, \mathcal{A}_{\nu}^{(-)}[\phi]\right\}\left(-2\left\{\mathcal{M}^{\rho^{\prime} \rho}, \delta g_{0}^{\rho^{\prime} \nu}\right\}+\left\{\mathcal{M}^{\mu \nu}, \delta g_{0}^{\rho \mu}\right\}+2 \delta g_{0}^{\rho \nu}\right) \\
& =-\frac{2}{R^{2}} \int\left\{t_{\rho}, \mathcal{A}_{\nu}^{(-)}[\phi]\right\}\left(-\frac{1}{2}\left(\left\{\mathcal{M}^{\rho^{\prime} \rho}, \delta g_{0}^{\rho^{\prime} \nu}\right\}+\left\{\mathcal{M}^{\rho^{\prime} \nu}, \delta g_{0}^{\rho^{\prime} \rho}\right\}\right)\right. \\
& \left.\quad-\frac{3}{2}\left(\left\{\mathcal{M}^{\rho^{\prime} \rho}, \delta g_{0}^{\rho^{\prime} \nu}\right\}-\left\{\mathcal{M}^{\rho^{\prime} \nu}, \delta g_{0}^{\rho^{\prime} \rho}\right\}\right)+2 \delta g_{0}^{\rho \nu}\right) \\
= & \frac{1}{R^{2}} \int 3 F_{\rho \nu}\left[\mathcal{A}^{(-)}\right]\left\{\mathcal{M}^{\mu \rho}, \delta g_{0}^{\mu \nu}\right\}-h_{\rho \nu}^{(g)}\left\{\mathcal{M}^{\mu \nu}, \delta g_{0}^{\rho \mu}\right\}+2 h_{\mu \nu}^{(g)} \delta g_{0}^{\mu \nu} \\
= & \frac{1}{R^{2}} \int 3 \delta g_{0}^{\mu \nu}\left\{\mathcal{M}^{\mu \rho}, \delta_{\phi} f_{\rho \nu}\right\}+\delta g_{0}^{\rho \mu}\left\{\mathcal{M}^{\mu \nu}, h_{\nu \rho}^{(g)}[\phi]\right\}+2 \delta g_{0}^{\mu \nu} h_{\mu \nu}^{(g)}[\phi]
\end{aligned}
$$

With the additional action terms (6.29) and their variations (6.32), $\Upsilon_{1}$ can be written as

$$
\begin{aligned}
\Upsilon_{1} & =\frac{1}{R^{2}} \int\left(3 \delta g_{0}^{\mu \nu}\left\{\mathcal{M}^{\mu \rho}, \delta_{\phi} f_{\rho \nu}\right\}+\delta g_{0}^{\rho \mu}\left\{\mathcal{M}^{\mu \nu}, h_{\nu \rho}^{(g)}[\phi]\right\}+2 \delta g_{0}^{\mu \nu} h_{\mu \nu}^{(g)}[\phi]\right) \\
& =\frac{1}{R^{2}} \int 3 \delta g_{0}^{\mu \nu}\left\{\mathcal{M}^{\mu \rho}, \delta_{\phi} f_{\rho \nu}\right\}+\frac{1}{2 R^{2}} \delta_{\phi} S_{4}+\frac{1}{R^{2}} \delta_{\phi} S_{3} .
\end{aligned}
$$

The first term in (A.3) can be re-written by noting that

$$
\begin{aligned}
-\int h^{\mu \nu}\left\{\mathcal{M}^{\nu \rho}, f_{\rho \mu}\left[\mathcal{A}^{(g)}\right]\right\}= & -\int\left\{x^{\mu}, A^{\nu}\right\}_{-}\left\{\mathcal{M}^{\nu \rho},\left\{x^{\mu}, A_{(g)}^{\rho}\right\}_{-}\right\} \\
& -\left\{x^{\nu}, A^{\mu}\right\}_{-}\left\{\mathcal{M}^{\nu \rho},\left\{x^{\rho}, A_{(g)}^{\mu}\right\}_{-}\right\} \\
& +\left\{x^{\nu}, A^{\mu}\right\}_{-}\left\{\mathcal{M}^{\nu \rho},\left\{x^{\mu}, A_{(g)}^{\rho}\right\}_{-}\right\} \\
& -\left\{x^{\mu}, A^{\nu}\right\}_{-}\left\{\mathcal{M}^{\nu \rho},\left\{x^{\rho}, A_{(g)}^{\mu}\right\}_{-}\right\} \\
= & \frac{1}{2} \delta_{\phi} S_{M 3}+\int\left\{\mathcal{M}^{\nu \rho},\left\{x^{\nu}, A^{\mu}\right\}_{-}\right\}\left\{x^{\mu}, A_{(g)}^{\rho}\right\}_{-} \\
& +\int\left\{x^{\mu}, A^{\nu}\right\}_{-}\left\{\mathcal{M}^{\nu \rho},\left\{x^{\rho}, A_{(g)}^{\mu}\right\}_{-}\right\} .
\end{aligned}
$$


Applying (A.33) twice yields

$$
\begin{aligned}
-\int h^{\mu \nu}\left\{\mathcal{M}^{\nu \rho}, f_{\rho \mu}\left[\mathcal{A}^{(g)}\right]\right\}= & \frac{1}{2} \delta_{\phi} S_{M 3}+\int\left(\left\{x^{\rho}, A^{\mu}\right\}_{-}+R\left\{t^{\rho}, D^{-} A^{\mu}\right\}\right)\left\{x^{\mu}, A_{(g)}^{\rho}\right\}_{-} \\
& -\int\left\{x^{\mu}, A^{\nu}\right\}_{-}\left(\left\{x^{\nu}, A_{(g)}^{\mu}\right\}_{-}+R\left\{t^{\nu}, D^{-} A_{(g)}^{\mu}\right\}\right) \\
= & \frac{1}{2} \delta_{\phi} S_{M 3}+R \int\left(\left\{t^{\rho}, D^{-} A^{\mu}\right\}\left\{x^{\mu}, A_{(g)}^{\rho}\right\}_{-}-\left\{x^{\mu}, A^{\nu}\right\}_{-}\left\{t^{\nu}, D^{-} A_{(g)}^{\mu}\right\}\right) \\
= & \frac{1}{2} \delta_{\phi} S_{M 3}-R \int\left(\left(D^{-} A^{\mu}\right)\left\{x^{\mu},\left\{t_{\rho}, A_{(g)}^{\rho}\right\}\right\}_{-}-\left\{x^{\mu},\left\{t_{\nu}, A^{\nu}\right\}\right\}_{-}\left(D^{-} A_{(g)}^{\mu}\right)\right) \\
= & \frac{1}{2} \delta_{\phi} S_{M 3}+R \int\left(\left\{x^{\mu}, D^{-} A^{\mu}\right\}_{+}\left\{t_{\rho}, A_{(g)}^{\rho}\right\}-\left\{t_{\nu}, A^{\nu}\right\}\left\{x^{\mu}, D^{-} A_{(g)}^{\mu}\right\}_{+}\right) \\
= & \frac{1}{2} \delta S_{M 3}+R \int\left(D^{+}\left(\left\{x^{\mu}, A^{\mu}\right\}_{-}\right)\left\{t_{\rho}, A_{(g)}^{\rho}\right\}-D^{+}\left\{x^{\mu}, A_{(g)}^{\mu}\right\}_{-}\left\{t_{\nu}, A^{\nu}\right\}\right) \\
= & \frac{1}{2} \delta S_{M 3}-R \int\left(\left\{x^{\mu}, A^{\mu}\right\}_{-} D^{-}\left(\left\{t_{\rho}, A_{(g)}^{\rho}\right\}\right)-\left\{x^{\mu}, A_{(g)}^{\mu}\right\}_{-} D^{-}\left(\left\{t_{\nu}, A^{\nu}\right\}\right)\right) \\
= & \frac{1}{2} \delta S_{M 3}-\delta_{\phi} S_{g f 2}+2 R \int\left(\left\{x^{\mu}, A_{(g)}^{\mu}\right\}_{-} D^{-}\left(\left\{t_{\nu}, A^{\nu}\right\}\right)\right)
\end{aligned}
$$

where we used

$$
\left\{x^{\mu}, D^{-} A^{\mu}\right\}_{+}=D^{+}\left(\left\{x^{\mu}, A^{\mu}\right\}_{-}\right)-\left\{D^{+} x^{\mu}, A^{\mu}\right\}_{-} .
$$

Now $\Upsilon_{1}$ needs

$$
\delta g_{0}^{\rho \mu}\left\{\mathcal{M}^{\mu \nu}, \delta_{\phi} f_{\nu \rho}\right\}=h^{\rho \mu}\left\{\mathcal{M}^{\mu \nu}, \delta_{\phi} f_{\nu \rho}\right\}-\frac{1}{4} h\left\{\mathcal{M}^{\mu \nu}, \delta_{\phi} f_{\nu \mu}\right\}
$$

The first term was just evaluated above, and the last term is

$$
\begin{aligned}
\int h\left\{\mathcal{M}^{\mu \nu}, \delta_{\phi} f_{\nu \mu}\right\} & =\int h\left\{\mathcal{M}^{\mu \nu},\left\{x_{\nu}, \mathcal{A}_{\mu}^{(g)}[\phi]\right\}_{-}-\left\{x_{\mu}, \mathcal{A}_{\nu}^{(g)}[\phi]\right\}_{-}\right\} \\
& =2 \int h\left\{\mathcal{M}^{\mu \nu},\left\{x_{\nu}, \mathcal{A}_{\mu}^{(g)}[\phi]\right\}_{-}\right\} \\
& =2 \int h\left(\left\{x^{\mu}, \mathcal{A}_{\mu}^{(g)}[\phi]\right\}_{-}+R\left\{t^{\mu}, D^{-} \mathcal{A}_{\mu}^{(g)}[\phi]\right\}\right) \\
& =-2 R \int \tilde{h} D^{-}\left(\left\{t^{\mu}, \mathcal{A}_{\mu}^{(g)}[\phi]\right\}\right) \\
& =-4 R \int\left\{x^{\nu}, \mathcal{A}_{\nu}\right\}_{-} D^{-}\left\{t^{\mu}, \mathcal{A}_{\mu}^{(g)}[\phi]\right\}
\end{aligned}
$$

using again (A.33) and

$$
\begin{aligned}
\left\{t^{\mu}, D^{-} \mathcal{A}_{\mu}\right\} & =D^{-}\left\{t^{\mu}, \mathcal{A}_{\mu}\right\}-\left\{D^{-} t^{\mu}, \mathcal{A}_{\mu}\right\} \\
& =D^{-}\left\{t^{\mu}, \mathcal{A}_{\mu}\right\}-\frac{1}{R}\left\{x^{\mu}, \mathcal{A}_{\mu}\right\}_{-} .
\end{aligned}
$$


Therefore

$$
\begin{aligned}
\int \delta g_{0}^{\rho \mu}\left\{\mathcal{M}^{\mu \nu}, \delta_{\phi} f_{\nu \rho}\right\}= & \int h^{\rho \mu}\left\{\mathcal{M}^{\mu \nu}, \delta_{\phi} f_{\nu \rho}\right\}-\int \frac{1}{4} h\left\{\mathcal{M}^{\mu \nu}, \delta_{\phi} f_{\nu \mu}\right\} \\
= & -\frac{1}{2} \delta S_{M 3}+\delta_{\phi} S_{g f 2}-2 R \int\left(\left\{x^{\mu}, A_{(g)}^{\mu}\right\}_{-} D^{-}\left(\left\{t_{\nu}, A^{\nu}\right\}\right)\right) \\
& +R \int\left\{x_{\nu}, \mathcal{A}^{\nu}\right\}_{-} D^{-}\left\{t_{\mu}, \mathcal{A}_{(g)}^{\mu}[\phi]\right\} \\
= & -\frac{1}{2} \delta S_{M 3}+2 \delta_{\phi} S_{g f 2}-3 R \int\left\{x_{\mu}, A_{(g)}^{\mu}\right\}_{-} D^{-}\left(\left\{t_{\nu}, A^{\nu}\right\}\right) \\
= & -\frac{1}{2} \delta S_{M 3}+2 \delta_{\phi} S_{g f 2}-3 \int D^{-} \phi D^{-}\left(\left\{t_{\nu}, A^{\nu}\right\}\right) .
\end{aligned}
$$

The last term is non-vanishing only for $\phi=D^{+} \phi^{0}$, and only the spin 0 mode $\mathcal{A}^{\mu}=$ $\mathcal{A}^{(+)}\left[\psi^{0}\right]=\left\{x^{\mu}, \psi^{0}\right\}$ for $\psi^{0} \in \mathcal{C}^{0}$ can contribute (since $\left\{t^{\nu}, A_{\nu}^{(-)}\left[\phi^{(2)}\right]\right\}=0$ ). Then

$$
D^{-}\left(\left\{t^{\nu}, \mathcal{A}_{\nu}^{(g)}\left[\phi^{0}\right]\right\}\right)=D^{-}\left(\square \phi^{0}\right)=\square D^{-} \phi^{0}
$$

so that the last term can be written as gauge variation (6.32f) of the action (6.29f). Consequently, we are lead to

$$
\begin{aligned}
\Upsilon_{1}= & \frac{1}{R^{2}} \int 3 \delta g_{0}^{\mu \nu}\left\{\mathcal{M}^{\mu \rho}, \delta_{\phi} f_{\rho \nu}\right\}+\frac{1}{2 R^{2}} \delta_{\phi} S_{4}+\frac{1}{R^{2}} \delta_{\phi} S_{3} \\
= & \frac{3}{R^{2}}\left(-\frac{1}{2} \delta S_{M 3}+2 \delta_{\phi} S_{g f 2}-3 R \int\left(\left\{x_{\mu}, A_{(g)}^{\mu}\right\}_{-} D^{-}\left(\left\{t_{\nu}, A^{\nu}\right\}\right)\right)\right) \\
& \quad+\frac{1}{2 R^{2}} \delta_{\phi} S_{4}+\frac{1}{R^{2}} \delta_{\phi} S_{3} \\
= & -\frac{3}{2 R^{2}} \delta S_{M 3}+\frac{6}{R^{2}} \delta_{\phi} S_{g f 2}-\frac{9}{2 R^{2}} \delta_{\phi} S_{g f 3}+\frac{1}{2 R^{2}} \delta_{\phi} S_{4}+\frac{1}{R^{2}} \delta_{\phi} S_{3} .
\end{aligned}
$$

Lastly, the conformal metric fluctuations contribute using (A.19) as follows:

$$
\begin{aligned}
\Upsilon_{2} & =-\frac{1}{3} \int \mathcal{A}_{\nu}^{(-)}[\phi]\left\{t_{\mu}, \mathcal{G}_{(\text {lin) }}^{\mu \nu}\left[h \eta_{\alpha \beta}\right]\right\} \\
& =-\frac{1}{2 R^{2}} \int \mathcal{A}_{\nu}^{(-)}[\phi]\left(\left\{t_{\nu}, h\right\}-\frac{1}{r^{2}} \tilde{\mathcal{I}}\left\{t_{\mu}, h\right\}\right) \\
& =-\frac{1}{2 R^{2}} \int\left(\mathcal{A}_{\nu}^{(-)}[\phi]\left\{t_{\nu}, h\right\}-\frac{1}{r^{2}} \tilde{\mathcal{I}}\left(\mathcal{A}_{\nu}^{(-)}[\phi]\right)\left\{t_{\mu}, h\right\}\right) \\
& =-\frac{1}{2 R^{2}} \int\left(\mathcal{A}_{\nu}^{(-)}[\phi]\left\{t_{\nu}, h\right\}-\frac{1}{r^{2}}\left(r^{2} \mathcal{A}^{\mu(-)}[\phi]+r^{2} R\left\{t^{\mu}, D^{-} \phi\right\}\right)\left\{t_{\mu}, h\right\}\right) \\
& =\frac{1}{2 R^{2}} \int R\left\{t^{\mu}, D^{-} \phi\right\}\left\{t_{\mu}, h\right\} \\
& =\frac{1}{2 R^{2}} \int R D^{-} \phi^{(s)} \square h=\frac{1}{12} \int h^{(g)} \square h \\
& =\frac{1}{24} \delta_{\phi} S_{h},
\end{aligned}
$$

using (A.33) and (6.8b). 


\section{A.2 Conservation law}

Using the definitions (6.16), we compute first for any trace-less metric $\delta g_{0}^{\mu \nu}$

$$
\begin{aligned}
2\left\{t_{\mu}, \mathcal{R}_{(\mathrm{lin})}^{\mu \nu}\left[\delta g_{0}\right]\right\}= & \left\{t_{\mu}, \square \delta g_{0}^{\mu \nu}\right\}-\square\left\{t_{\rho}, \delta g_{0}^{\rho \nu}\right\}+\left\{t_{\mu},\left\{t^{\nu},\left\{t_{\rho}, \delta g_{0}^{\rho \mu}\right\}\right\}\right\} \\
= & \left\{t_{\mu}, \square \delta g_{0}^{\mu \nu}\right\}-\square\left\{t_{\rho}, \delta g_{0}^{\rho \nu}\right\}-\frac{1}{r^{2} R^{2}}\left\{\theta^{\mu \nu},\left\{t_{\rho}, \delta g_{0}^{\rho \mu}\right\}\right\} \\
& +\left\{t^{\nu},\left\{t_{\mu},\left\{t_{\rho}, \delta g_{0}^{\rho \mu}\right\}\right\}\right\} .
\end{aligned}
$$

Now we use

$$
\begin{aligned}
\square\left(\left\{t_{\rho}, \delta g_{0}^{\rho \nu}\right\}\right) & =\left\{t_{\rho},\left(\square+\frac{3}{R^{2}}\right) \delta g_{0}^{\rho \nu}\right\}-\frac{2}{r^{2} R^{2}}\left\{\theta^{\rho \rho^{\prime}},\left\{t_{\rho^{\prime}}, \delta g_{0}^{\rho \nu}\right\}\right\} \\
& =\left\{t_{\rho},\left(\square+\frac{3}{R^{2}}\right) \delta g_{0}^{\rho \nu}\right\}-\frac{2}{r^{2} R^{2}}\left(\left\{t_{\rho^{\prime}},\left\{\theta^{\rho \rho^{\prime}}, \delta g_{0}^{\rho \nu}\right\}\right\}+\left\{\left\{\theta^{\rho \rho^{\prime}}, t_{\rho^{\prime}}\right\}, \delta g_{0}^{\rho \nu}\right\}\right) \\
& =\left\{t_{\rho},\left(\square+\frac{3}{R^{2}}\right) \delta g_{0}^{\rho \nu}\right\}-\frac{2}{r^{2} R^{2}}\left(\left\{t_{\rho^{\prime}},\left\{\theta^{\rho \rho^{\prime}}, \delta g_{0}^{\rho \nu}\right\}\right\}+3 r^{2}\left\{t^{\rho}, \delta g_{0}^{\rho \nu}\right\}\right) \\
& =\left\{t_{\rho}, \square \delta g_{0}^{\rho \nu}\right\}-\frac{2}{r^{2} R^{2}}\left(\left\{t_{\rho^{\prime}},\left\{\theta^{\rho \rho^{\prime}}, \delta g_{0}^{\rho \nu}\right\}\right\}+\frac{3}{2} r^{2}\left\{t^{\rho}, \delta g_{0}^{\rho \nu}\right\}\right)
\end{aligned}
$$

due to (5.27). Hence

$$
\left\{t_{\rho}, \square \delta g_{0}^{\rho \nu}\right\}-\square\left(\left\{t_{\rho}, \delta g_{0}^{\rho \nu}\right\}\right)=\frac{2}{r^{2} R^{2}}\left\{t_{\rho^{\prime}},\left\{\theta^{\rho \rho^{\prime}}, \delta g_{0}^{\rho \nu}\right\}\right\}+\frac{3}{R^{2}}\left\{t_{\rho}, \delta g_{0}^{\rho \nu}\right\}
$$

Therefore

$$
\begin{aligned}
2\left\{t_{\mu}, \mathcal{R}_{(\mathrm{lin})}^{\mu \nu}\left[\delta g_{0}\right]\right\}= & \frac{1}{r^{2} R^{2}}\left(2\left\{t_{\rho^{\prime}},\left\{\theta^{\rho \rho^{\prime}}, \delta g_{0}^{\rho \nu}\right\}\right\}-\left\{\theta^{\mu \nu},\left\{t_{\rho}, \delta g_{0}^{\rho \mu}\right\}\right\}\right)+\frac{3}{R^{2}}\left\{t^{\rho}, \delta g_{0}^{\rho \nu}\right\} \\
& +\left\{t^{\nu},\left\{t_{\mu},\left\{t_{\rho}, \delta g_{0}^{\rho \mu}\right\}\right\}\right\}
\end{aligned}
$$

The second term can be written as

$$
\begin{aligned}
\left\{\theta^{\mu \nu},\left\{t_{\rho}, \delta g_{0}^{\rho \mu}\right\}\right\} & =\left\{\left\{\theta^{\mu \nu}, t_{\rho}\right\}, \delta g_{0}^{\rho \mu}\right\}+\left\{t_{\rho},\left\{\theta^{\mu \nu}, \delta g_{0}^{\rho \mu}\right\}\right\} \\
& =-r^{2}\left\{\eta^{\mu \rho} t^{\nu}-\eta^{\nu \rho} t^{\mu}, \delta g_{0}^{\rho \mu}\right\}+\left\{t_{\rho},\left\{\theta^{\mu \nu}, \delta g_{0}^{\rho \mu}\right\}\right\} \\
& =r^{2}\left\{t^{\mu}, \delta g_{0}^{\nu \mu}\right\}+\left\{t_{\rho},\left\{\theta^{\mu \nu}, \delta g_{0}^{\rho \mu}\right\}\right\}
\end{aligned}
$$

using $\delta g_{0}=0$. Hence

$$
\begin{aligned}
2\left\{t_{\mu}, \mathcal{R}_{(\text {lin })}^{\mu \nu}\left[\delta g_{0}\right]\right\}= & \frac{1}{r^{2} R^{2}}\left(2\left\{t_{\rho^{\prime}},\left\{\theta^{\rho \rho^{\prime}}, \delta g_{0}^{\rho \nu}\right\}\right\}-\left\{t_{\rho},\left\{\theta^{\mu \nu}, \delta g_{0}^{\rho \mu}\right\}\right\}\right)+\frac{2}{R^{2}}\left\{t_{\rho}, \delta g_{0}^{\rho \nu}\right\} \\
& +\left\{t^{\nu},\left\{t_{\mu},\left\{t_{\rho}, \delta g_{0}^{\rho \mu}\right\}\right\}\right\}
\end{aligned}
$$

Similarly $\mathcal{R}_{(\operatorname{lin})}\left[\delta g_{0}^{\mu \nu}\right]=\left\{t_{\mu},\left\{t_{\rho}, \delta g_{0}^{\rho \mu}\right\}\right\}$, and (6.16) gives

$$
\begin{aligned}
2\left\{t_{\mu}, \mathcal{G}_{(\text {lin })}^{\mu \nu}\left[\delta g_{0}\right]\right\} & =2\left\{t_{\mu}, \mathcal{R}_{(\operatorname{lin})}^{\mu \nu}\right\}-\left\{t_{\nu}, \mathcal{R}_{(\text {lin })}\right\} \\
& =\frac{1}{r^{2} R^{2}}\left(2\left\{t_{\rho^{\prime}},\left\{\theta^{\rho \rho^{\prime}}, \delta g_{0}^{\rho \nu}\right\}\right\}-\left\{t_{\rho},\left\{\theta^{\mu \nu}, \delta g_{0}^{\rho \mu}\right\}\right\}\right)+\frac{2}{R^{2}}\left\{t_{\rho}, \delta g_{0}^{\rho \nu}\right\} .
\end{aligned}
$$


Now consider trace contributions to the metric

$$
\mathcal{G}_{(\operatorname{lin})}^{\mu \nu}\left[h \eta_{\alpha \beta}\right]=-\square h \eta^{\mu \nu}-\frac{1}{2}\left(\left\{t_{\mu},\left\{t_{\nu}, h\right\}\right\}+(\mu \leftrightarrow \nu)\right) .
$$

These contribute as follows

$$
\begin{aligned}
\left\{t_{\mu}, \mathcal{G}_{\text {(lin) }}^{\mu \nu}[h \eta]\right\} & =-\left\{t_{\nu}, \square h\right\}-\frac{1}{2}\left\{t_{\mu},\left\{t_{\mu},\left\{t_{\nu}, h\right\}\right\}\right\}-\frac{1}{2}\left\{t_{\mu},\left\{t_{\nu},\left\{t_{\mu}, h\right\}\right\}\right\} \\
& =-\left\{t_{\nu}, \square h\right\}+\frac{1}{2} \square\left\{t_{\nu}, h\right\}+\frac{1}{2}\left\{t_{\nu}, \square h\right\}-\frac{1}{2 r^{2} R^{2}}\left\{\theta^{\nu \mu},\left\{t_{\mu}, h\right\}\right\} \\
& =-\left\{t_{\nu}, \square h\right\}+\frac{1}{2}\left(\mathcal{D}^{2}-\frac{2}{r^{2} R^{2}} \tilde{\mathcal{I}}\right)\left\{t_{\nu}, h\right\}+\frac{1}{2}\left\{t_{\nu}, \square h\right\}-\frac{1}{2 r^{2} R^{2}} \tilde{\mathcal{I}}\left\{t_{\mu}, h\right\} \\
& =-\left\{t_{\nu}, \square h\right\}-\frac{1}{r^{2} R^{2}} \tilde{\mathcal{I}}\left\{t_{\nu}, h\right\}+\frac{1}{2}\left\{t_{\nu},\left(\square+\frac{3}{R^{2}}\right) h\right\}+\frac{1}{2}\left\{t_{\nu}, \square h\right\}-\frac{1}{2 r^{2} R^{2}} \tilde{\mathcal{I}}\left\{t_{\mu}, h\right\} \\
& =\frac{3}{2 R^{2}}\left\{t_{\nu}, h\right\}-\frac{3}{2 r^{2} R^{2}} \tilde{\mathcal{I}}\left\{t_{\mu}, h\right\}
\end{aligned}
$$

\section{A.3 Time-like mode}

Suppose there exists an extra on-shell gauge-fixed solution to $\left(\mathcal{D}^{2}+\frac{1}{2} \mu^{2}\right) \mathcal{B}^{(\tau)}=0$ of the form

$$
\mathcal{B}^{(\tau)}\left[\phi^{(s)}\right]:=\mathcal{A}^{(\tau)}\left[\phi^{(s)}\right]+\left\{t^{\mu}, \chi^{(s)}\right\}+\sum_{ \pm} \mathcal{B}^{( \pm)}\left[\phi_{ \pm}\right] \quad \in \mathcal{C}^{s}
$$

where $\chi$ is determined by $0=\mathcal{G}\left(\mathcal{B}^{(\tau)}\right) \equiv\left\{t^{\mu}, \mathcal{B}_{\mu}^{(\tau)}\right\}$; hence, by using (3.40), this reduces to

$$
\sinh (\eta)(4+s+\tau) \phi^{(s)}=\square \chi^{(s)} .
$$

The on-shell condition gives

$$
\begin{aligned}
0 & =\left(\mathcal{D}^{2}+\frac{1}{2} \mu^{2}\right) \mathcal{B}_{\mu}^{(\tau)}[\phi]=x_{\mu}\left(\square+\frac{4}{R^{2}}\right) \phi+2 \mho_{\mu} \phi+\left\{t^{\mu}, \square \chi\right\}+\sum_{ \pm} \mathcal{B}^{( \pm)}\left[(\square+\ldots) \phi_{ \pm}\right] \\
& =x_{\mu}\left(\square+\frac{4}{R^{2}}\right) \phi+2 ð_{\mu} \phi+\left\{t^{\mu}, \sinh (\eta)(4+s+\tau) \phi\right\}+\sum_{ \pm} \mathcal{B}^{( \pm)}\left[(\square+\ldots) \phi_{ \pm}\right] \quad(\mathrm{A} .22
\end{aligned}
$$

Taking $\left\{t^{\mu}, \cdot\right\}$ of that expression yields

$$
\begin{aligned}
0 & =\left\{t^{\mu}, x_{\mu}\left(\square+\frac{1}{R^{2}}\right) \phi^{(s)}\right\}+2\left\{t^{\mu}, \partial_{\mu} \phi^{(s)}\right\}-\square\left(\sinh (\eta)(4+s+\tau) \phi^{(s)}\right) \\
& =\frac{x_{4}}{R}(4+s+\tau)\left(\left(\square+\frac{1}{R^{2}}\right) \phi\right)+2\left\{t^{\mu}, \partial_{\mu} \phi^{(s)}\right\}-\frac{1}{R} \square\left(x_{4}(4+s+\tau) \phi\right) \\
& =\frac{x_{4}}{R^{3}}(4+s+\tau) \phi+2\left\{t^{\mu}, \partial_{\mu} \phi^{(s)}\right\}-\frac{4}{R^{3}} x_{4}(4+s+\tau) \phi+\frac{2}{R}\left\{t_{\mu}, x_{4}\right\}\left\{t^{\mu},(4+s+\tau) \phi\right\} \\
& =2\left\{t^{\mu}, \partial_{\mu} \phi^{(s)}\right\}-\frac{3 x_{4}}{R^{3}}(4+s+\tau) \phi-\frac{2 x_{4}}{R^{3}}(s+\tau)((4+s+\tau) \phi)
\end{aligned}
$$

since the $\mathcal{B}^{( \pm)}$are gauge-fixed, and we used (A.21) and $\square x_{4}=\frac{4}{R^{2}} x^{4}$. This provides some (Lorentz-violating) on-shell condition for $\phi$. However, (A.22) states that $x_{\mu}\left(\square+\frac{4}{R^{2}}\right) \phi+$ $2 \widetilde{\partial}_{\mu} \phi+\left\{t^{\mu}, \sinh (\eta)(4+s+\tau) \phi\right\}$ is a linear combination of the two $\mathcal{B}_{\mu}^{( \pm)}$modes. This implies that there is another, independent equation for $\phi$, which strongly suggests that there is no generic solution. Unfortunately we cannot provide a formal proof here. 


\section{A.4 Group theory and useful identities}

Consider

$$
\begin{aligned}
{\left[C^{2}[\mathfrak{s o}(4,1)], \mathcal{M}^{45}\right] } & =-\mathrm{i}\left(\mathcal{M}_{b 4} \mathcal{M}^{b 5}+\mathcal{M}^{b 5} \mathcal{M}_{b 4}\right) \\
& =-2 \mathrm{i} \mathcal{M}_{b 4} \mathcal{M}^{b 5}-\mathrm{i}\left[\mathcal{M}^{b 5}, \mathcal{M}_{b 4}\right] \\
& =-2 \mathrm{i} \mathcal{M}_{b 4} \mathcal{M}^{b 5}+4 \mathcal{M}^{54}=-2 \mathrm{i} \frac{R}{r} T_{\mu} X^{\mu}+4 \mathcal{M}^{54},
\end{aligned}
$$

such that

$$
C^{2}[\mathfrak{s o}(4,1)] \mathcal{M}^{45}=\mathcal{M}^{45}\left(C^{2}[\mathfrak{s o}(4,1)]-4\right)-2 \mathrm{i} \frac{R}{r} T_{\mu} X^{\mu}
$$

This holds in any representation, in particular in the adjoint acting on functions. Thus

$$
\begin{aligned}
-\mathrm{i} \frac{2 R}{r} \operatorname{Tr}\left(\phi^{\prime}\left[T_{\mu},\left[X^{\mu}, \phi\right]\right]\right) & =\operatorname{Tr} \phi^{\prime}\left(C^{2 \mathrm{ad}}[\mathfrak{s o}(4,1)]\left[\mathcal{M}^{45}, \phi\right]-\left[\mathcal{M}^{45},\left(C^{2 \mathrm{ad}}[\mathfrak{s o}(4,1)]-4\right) \phi\right]\right) \\
& =\left(C^{2}\left[\phi^{\prime}\right]-C^{2}[\phi]+4\right) \operatorname{Tr} \phi^{\prime}\left[\mathcal{M}^{45}, \phi\right] .
\end{aligned}
$$

Similarly,

$$
\begin{aligned}
{\left[T_{\mu} T^{\mu}, \mathcal{M}^{45}\right] } & =T^{\mu}\left[T_{\mu}, \mathcal{M}^{45}\right]+\left[T_{\mu}, \mathcal{M}^{45}\right] T^{\mu} \\
& =-\mathrm{i} \frac{1}{r R}\left(T^{\mu} X^{\mu}+X^{\mu} T^{\mu}\right) \\
& =-\mathrm{i} \frac{2}{r R} T^{\mu} X^{\mu}-\frac{4}{r R^{2}} X^{4}
\end{aligned}
$$

in any representation. ${ }^{28}$ This is consistent with (3.5). In particular, from

$$
\left[C^{2}[\mathfrak{s o}(4,1)], \mathcal{M}^{45}\right]=\frac{1}{2}\left[\mathcal{S}^{2}, \mathcal{M}^{45}\right]=R^{2}\left[T_{\mu} T^{\mu}, \mathcal{M}^{45}\right]
$$

(since $\left.\left[C^{2}[\mathfrak{s o}(3,1)], \mathcal{M}^{45}\right]=0\right)$ we obtain in the adjoint representation

$$
-\mathrm{i} \frac{2 R}{r} \operatorname{Tr} \phi^{\prime}\left[T_{\mu},\left[X^{\mu}, \phi\right]\right]=\operatorname{Tr} \phi^{\prime}\left(R^{2} \square\left[\mathcal{M}^{45}, \phi\right]-\left[\mathcal{M}^{45},\left(R^{2} \square-4\right) \phi\right]\right) .
$$

Identities for $\mathcal{I}\left(\left\{x^{\mu}, \phi\right\}\right)$. Next, recall the following result of [40]

$$
2 \tilde{\mathcal{I}}^{(5)}\left(\left\{x_{a}, \phi\right\}\right)=2\left\{\theta^{a b},\left\{x_{b}, \phi\right\}\right\}=r^{2}\left(\frac{1}{2} \mathcal{S}^{2}-s(s+1)+4\right)\left(\left\{x_{a}, \phi\right\}\right), \quad a=0, \ldots, 4
$$

and in particular

$$
\begin{aligned}
& \tilde{\mathcal{I}}^{(5)}\left(\left\{x_{a}, \phi^{(s)}\right\}_{+}\right)=r^{2}(s+3)\left\{x_{a}, \phi^{(2)}\right\}_{+} \\
& \tilde{\mathcal{I}}^{(5)}\left(\left\{x_{a}, \phi^{(s)}\right\}_{-}\right)=r^{2}(-s+2)\left\{x_{a}, \phi^{(2)}\right\}_{-}
\end{aligned}
$$

\footnotetext{
${ }^{28}$ Note that $(2.35)$ only holds on $\mathcal{H}_{n}$, but not e.g. in the adjoint representation.
} 
which then implies

$$
\tilde{\mathcal{I}}\left(\left\{x^{\mu}, \phi^{(s)}\right\}\right)=\frac{r^{2}}{2}\left(\frac{1}{2} \mathcal{S}^{2}-s(s+1)+4\right)\left\{x^{\mu}, \phi^{(s)}\right\}+r^{2} R\left\{t^{\mu},\left\{x^{4}, \phi^{(s)}\right\}\right\} .
$$

For the $\mathcal{A}^{( \pm)}$modes, this gives

$$
\begin{aligned}
& \tilde{\mathcal{I}}\left(\mathcal{A}^{\mu(+)}\right)=r^{2}(s+3) \mathcal{A}^{\mu(+)}+r^{2} R\left\{t^{\mu}, D^{+} \phi^{(s)}\right\} \\
& \tilde{\mathcal{I}}\left(\mathcal{A}^{\mu(-)}\right)=r^{2}(-s+2) \mathcal{A}^{\mu(-)}+r^{2} R\left\{t^{\mu}, D^{-} \phi^{(s)}\right\} .
\end{aligned}
$$

Furthermore, evaluating the 4 component of (A.30) yields

$$
R\left\{t^{\mu},\left\{x_{\mu}, \phi^{(s)}\right\}\right\}=\frac{1}{2}\left(\frac{1}{2} \mathcal{S}^{2}-s(s+1)+4\right)\left\{x^{4}, \phi^{(s)}\right\},
$$

which is useful to understand the orthogonality. Using $\mathcal{S}^{2}=2 C^{2}[\mathfrak{s o}(4,1)]-C^{2}[\mathfrak{s o}(4,2)]$ as in (3.2), one can see that this relation is nothing but (A.26) in the adjoint representation. For the $\mathcal{A}^{( \pm)}$modes, this gives

$$
\begin{aligned}
& R\left\{t^{\mu}, \mathcal{A}_{\mu}^{(+)}\left[\phi^{(s)}\right]\right\}=(s+3)\left\{x^{4}, \phi^{(s)}\right\}_{+}, \\
& R\left\{t^{\mu}, \mathcal{A}_{\mu}^{(-)}\left[\phi^{(s)}\right]\right\}=(-s+2)\left\{x^{4}, \phi^{(s)}\right\}_{-} .
\end{aligned}
$$

Similarly, (A.29) gives along the same lines

$$
R\left\{t^{\mu},\left\{x_{\mu}, \phi^{(s)}\right\}\right\}=\frac{1}{2}\left(\left(R^{2} \square+4\right)\left\{x^{4}, \phi^{(s)}\right\}-\left\{x^{4}, R^{2} \square \phi^{(s)}\right\}\right)
$$

which immediately implies

$$
R\left\{t^{\mu}, \mathcal{A}_{\mu}^{( \pm)}\left[\phi^{(s)}\right]\right\}=\frac{1}{2}\left(\left(R^{2} \square+4\right)\left\{x^{4}, \phi^{(s)}\right\}_{ \pm}-\left\{x^{4}, R^{2} \square \phi^{(s)}\right\}_{ \pm}\right)
$$

and together with (A.35), we find

$$
\begin{aligned}
& \square D^{+} \phi^{(s)}=D^{+}\left(\square+\frac{2 s+2}{R^{2}}\right) \phi^{(s)}, \\
& \square D^{-} \phi^{(s)}=D^{-}\left(\square-\frac{2 s}{R^{2}}\right) \phi^{(s)} .
\end{aligned}
$$

This implies

$$
\square D^{+} D^{-} \phi^{(s)}=D^{+} D^{-} \square \phi^{(s)}, \quad \square D^{-} D^{+} \phi^{(s)}=D^{-} D^{+} \square \phi^{(s)}
$$

and

$$
\begin{aligned}
& \square^{-1} D^{+} \phi=D^{+}\left(\square+\frac{2 s+2}{R^{2}}\right)^{-1} \phi, \\
& \square^{-1} D^{-} \phi=D^{-}\left(\square-\frac{2 s}{R^{2}}\right)^{-1} \phi,
\end{aligned}
$$

provided the inverses on the right-hand-side exist. We finally note

$$
D^{-}\left\{x^{\nu}, \phi^{(2)}\right\}_{-}=\left\{x^{4},\left\{x_{\nu}, \phi^{(2)}\right\}_{-}\right\}_{-}=\left\{x^{\nu},\left\{x_{4}, \phi^{(2)}\right\}_{-}\right\}_{-}=\left\{x^{\nu}, D^{-} \phi^{(2)}\right\}_{-}
$$

since $\left\{t^{\nu}, \cdot\right\}$ respects the spin. 
$\mathcal{D}^{2}$ intertwiner for $\boldsymbol{h}_{\boldsymbol{\mu \nu}}$. To evaluate $\mathcal{R}_{(\text {lin) }}^{\mu \nu}\left[h^{\mu \nu}\right]$, it is useful to extend the definition (6.3) of $h^{\mu \nu}$ as follows:

$$
\begin{aligned}
& \mathcal{A} \mapsto h_{(4)}^{\mu \nu}[\mathcal{A}]=\left\{\mathcal{A}^{\mu}, x^{\nu}\right\}_{-}+(\mu \leftrightarrow \nu) \\
& \mathcal{A} \mapsto h_{(5)}^{a b}[\mathcal{A}]=\left\{\mathcal{A}^{a}, x^{b}\right\}_{-}+(a \leftrightarrow b)
\end{aligned}
$$

viewed as $\mathrm{SO}(3,1)$ and $\mathrm{SO}(4,1)$ intertwiners, respectively. Here we assume that $\mathcal{A}^{\mu}$ arises from $\mathcal{A}^{b}$ in $(5) \otimes(\mathrm{ad})$; this is the case for all physical fluctuation modes as discussed in section 5.3. Then the following the intertwiner property holds:

$$
C^{2}[\mathfrak{s o}(4,1)]^{(\mathrm{full})} h_{(5)}^{a b}[\mathcal{A}]=h_{(5)}^{a b}\left[C^{2}[\mathfrak{s o}(4,1)]^{(\text {full })} \mathcal{A}\right]
$$

and similarly for $\mathrm{SO}(3,1)$. The full Casimirs can be expressed in terms of $\tilde{\mathcal{I}}$ as explained in section 5.2 , and we obtain

$$
\begin{aligned}
& \left(C^{2}[\mathfrak{s o}(4,1)]^{(\mathrm{ad})}-2 r^{-2} \tilde{\mathcal{I}}^{(5)}+C_{(5)}^{2}\right) h_{(5)}^{a b}[\mathcal{A}]=h_{(5)}^{a b}\left[\left(C^{2}[\mathfrak{s o}(4,1)]^{(\mathrm{ad})}-2 r^{-2} \tilde{\mathcal{I}}^{(5)}+4\right) \mathcal{A}\right] \\
& \left(C^{2}[\mathfrak{s o}(3,1)]^{(\mathrm{ad})}-2 r^{-2} \tilde{\mathcal{I}}^{(4)}+C_{(4)}^{2}\right) h_{(4)}^{\mu \nu}[\mathcal{A}]=h_{(4)}^{\mu \nu}\left[\left(C^{2}[\mathfrak{s o}(3,1)]^{(\mathrm{ad})}-2 r^{-2} \tilde{\mathcal{I}}^{(4)}+3\right) \mathcal{A}\right]
\end{aligned}
$$

using (3.2), and $C_{(5)}^{2}:=C^{2}[\mathfrak{s o}(4,1)]^{(5) \otimes(5)}$ and $C_{(4)}^{2}:=C^{2}[\mathfrak{s o}(3,1)]^{(4) \otimes(4)}$ denotes the Casimirs acting on the indices. Here

$$
\tilde{\mathcal{I}}^{(5)} h_{(5)}^{\mu \nu}[\mathcal{A}]=\left\{\theta^{\mu a}, h_{(5)}^{a \nu}\right\}+\left\{\theta^{\nu a}, h_{(5)}^{\mu a}\right\} .
$$

Subtracting these for $h_{(5)}^{\mu \nu}=h_{(4)}^{\mu \nu}=h^{\mu \nu}$, we obtain

$$
\begin{aligned}
\left(R^{2} \square-2 r^{-2}\left(\tilde{\mathcal{I}}^{(5)}-\tilde{\mathcal{I}}^{(4)}\right)+C_{(5)}^{2}-C_{(4)}^{2}\right) h^{\mu \nu}[\mathcal{A}] & =h^{\mu \nu}\left[\left(R^{2} \square-2 r^{-2}\left(\tilde{\mathcal{I}}^{(5)}-\tilde{\mathcal{I}}^{(4)}\right)+1\right) \mathcal{A}\right] \\
\left(R^{2} \mathcal{D}^{2}-2 r^{-2} \tilde{\mathcal{I}}^{(5)}+C_{(5)}^{2}-C_{(4)}^{2}\right) h^{\mu \nu}[\mathcal{A}] & =h^{\mu \nu}\left[\left(R^{2} \mathcal{D}^{2}-2 r^{-2} \tilde{\mathcal{I}}^{(5)}+1\right) \mathcal{A}\right]
\end{aligned}
$$

where $\mathcal{D}^{2}=\square+\frac{2}{r^{2} R^{2}} \tilde{\mathcal{I}}^{(4)}$. To proceed, we need

$$
\begin{aligned}
\mathcal{I}^{(5)}\left\{\mathcal{A}^{\mu}, x^{\nu}\right\}_{-}-\left\{\mathcal{I}^{(5)} \mathcal{A}^{\mu}, x^{\nu}\right\}_{-} & =\left\{\theta^{\mu b},\left\{\mathcal{A}_{b}, x^{\nu}\right\}_{-}\right\}+\left\{\theta^{\nu b},\left\{\mathcal{A}^{\mu}, x_{b}\right\}_{-}\right\}-\left\{\left\{\theta^{\mu b}, \mathcal{A}_{b}\right\}, x^{\nu}\right\}_{-} \\
& =r^{2}\left\{\mathcal{A}^{\mu}, x^{\nu}\right\}_{-}+\left\{\theta^{\mu b},\left\{\mathcal{A}_{b}, x^{\nu}\right\}_{-}\right\}+\left\{x^{\nu},\left\{\theta^{\mu b}, \mathcal{A}_{b}\right\}\right\}_{-} \\
& =r^{2}\left\{\mathcal{A}^{\mu}, x^{\nu}\right\}_{-}+\left\{\mathcal{A}_{b},\left\{\theta^{\mu b}, x^{\nu}\right\}_{-}\right\} \\
& =r^{2}\left\{\mathcal{A}^{\mu}, x^{\nu}\right\}_{-}-r^{2}\left\{\mathcal{A}_{b}, x^{b}\right\}_{-} \eta_{\mu \nu}+r^{2}\left\{\mathcal{A}^{\nu}, x^{\mu}\right\}_{-}
\end{aligned}
$$

using (2.24), (A.31), and the Jacobi identity. This gives

$$
\mathcal{I}^{(5)} h^{\mu \nu}[\mathcal{A}]-h^{\mu \nu}\left[\tilde{\mathcal{I}}^{(5)} \mathcal{A}\right]=2 r^{2} h^{\mu \nu}[\mathcal{A}]-2 r^{2}\left\{\mathcal{A}_{b}, x^{b}\right\}_{-} \eta_{\mu \nu}
$$

and therefore

$$
\left(\mathcal{D}^{2}+\frac{1}{R^{2}}\left(C_{(5)}^{2}-C_{(4)}^{2}\right)\right) h^{\mu \nu}[\mathcal{A}]=h^{\mu \nu}\left[\left(\mathcal{D}^{2}+\frac{4}{R^{2}}\right) \mathcal{A}\right]+\frac{4}{R^{2}}\left\{\mathcal{A}_{b}, x^{b}\right\}_{-} \eta_{\mu \nu}
$$


hence

$$
\begin{aligned}
\mathcal{D}^{2} h^{\mu \nu}[\mathcal{A}] & =h^{\mu \nu}\left[\left(\mathcal{D}^{2}+\frac{6}{R^{2}}\right) \mathcal{A}\right]+\frac{2}{R^{2}} \eta_{\mu \nu}\left(-h_{(5)}[\mathcal{A}]+h_{(5)}^{44}[\mathcal{A}]\right) \\
& =h^{\mu \nu}\left[\mathcal{D}^{2} \mathcal{A}\right]+\frac{2}{R^{2}}\left(3 h^{\mu \nu}[\mathcal{A}]-\eta^{\mu \nu} h[\mathcal{A}]\right)
\end{aligned}
$$

(where $\left.h \equiv h_{(4)}\right)$, using $2\left\{\mathcal{A}_{b}, x^{b}\right\}_{-}=-h_{(5)}$ and

$$
\left(C_{(5)}^{2}-C_{(4)}^{2}\right) h^{\mu \nu}[\mathcal{A}]=-2 h^{\mu \nu}[\mathcal{A}]-2 \eta^{\mu \nu} h^{44}[\mathcal{A}]
$$

since $M_{(5)}^{\alpha 4} v_{\mu}=-i \delta_{\mu}^{\alpha} v^{4}$ using (5.20).

\section{A.5 Consistency checks}

Here, we check the implications of (5.38) for consistency. To begin with, (5.38) implies

$$
\begin{aligned}
\int & \mathcal{A}_{\mu}^{(+)}\left[D^{-} \phi^{(s)}\right] \tilde{\mathcal{I}} \mathcal{A}_{\mu}^{(-)}\left[D^{+} \phi^{(s)}\right] \\
& =\int \mathcal{A}_{\mu}^{(+)}\left[D^{-} \phi^{\prime(s)}\right]\left(r^{2}(-s+1) \mathcal{A}^{\mu(-)}\left[D^{+} \phi^{(s)}\right]+r^{2} R\left\{t^{\mu}, D^{-} D^{+} \phi^{(s)}\right\}\right) \\
& =\int \tilde{\mathcal{I}} \mathcal{A}_{\mu}^{(+)}\left[D^{-} \phi^{\prime(s)}\right] \mathcal{A}_{\mu}^{(-)}\left[D^{+} \phi^{(s)}\right] \\
& =\int\left(r^{2}(s+2) \mathcal{A}^{\mu(+)}\left[D^{-} \phi^{\prime(s)}\right]+r^{2} R\left\{t^{\mu}, D^{+} D^{-} \phi^{(s)}\right\}\right) \mathcal{A}_{\mu}^{(-)}\left[D^{+} \phi^{(s)}\right]
\end{aligned}
$$

using the fact that $\tilde{\mathcal{I}}$ is self-adjoint, see (5.15). From this equality, we learn that

$$
\begin{aligned}
\int & (-s+1) \mathcal{A}_{\mu}^{(+)}\left[D^{-} \phi^{\prime(s)}\right] \mathcal{A}^{\mu(-)}\left[D^{+} \phi^{(s)}\right]+R \mathcal{A}_{\mu}^{(+)}\left[D^{-} \phi^{\prime(s)}\right]\left\{t^{\mu}, D^{-} D^{+} \phi^{(s)}\right\} \\
= & \int(s+2) \mathcal{A}^{\mu(+)}\left[D^{-} \phi^{\prime(s)}\right] \mathcal{A}_{\mu}^{(-)}\left[D^{+} \phi^{(s)}\right]+R\left\{t^{\mu}, D^{+} D^{-} \phi^{\prime(s)}\right\} \mathcal{A}_{\mu}^{(-)}\left[D^{+} \phi^{(s)}\right] \\
& -(2 s+1) \int \mathcal{A}_{\mu}^{(+)}\left[D^{-} \phi^{\prime(s)}\right] \mathcal{A}^{\mu(-)}\left[D^{+} \phi^{(s)}\right]-R \int\left\{t^{\mu}, \mathcal{A}_{\mu}^{(+)}\left[D^{-} \phi^{\prime(s)}\right]\right\} D^{-} D^{+} \phi^{(s)} \\
= & -R \int D^{+} D^{-} \phi^{\prime(s)}\left\{t^{\mu}, \mathcal{A}_{\mu}^{(-)}\left[D^{+} \phi^{(s)}\right]\right\} \\
& -(2 s+1) \int \mathcal{A}_{\mu}^{(+)}\left[D^{-} \phi^{\prime(s)}\right] \mathcal{A}^{\mu(-)}\left[D^{+} \phi^{(s)}\right]-(s+2) \int D^{+} D^{-} \phi^{\prime(s)} D^{-} D^{+} \phi^{(s)} \\
= & -(-s+1) \int D^{+} D^{-} \phi^{\prime(s)} D^{-} D^{+} \phi^{(s)}
\end{aligned}
$$

such that

$$
-\int \mathcal{A}_{\mu}^{(+)}\left[D^{-} \phi^{\prime(s)}\right] \mathcal{A}^{\mu(-)}\left[D^{+} \phi^{(s)}\right]=\int D^{+} D^{-} \phi^{\prime(s)} D^{-} D^{+} \phi^{(s)} .
$$

This is the same as (5.58i), which provides a consistency test. 


\section{A.6 Relations for $\square_{H}$}

Consider the following intertwiner relation discussed in [40]:

$$
\begin{aligned}
r^{2} C^{2}[\mathfrak{s o}(4,1)]^{(\mathrm{full})} \mathcal{A}_{a}^{(-)}\left[\phi^{(s)}\right] & =-\left(\square_{H}+2 \mathcal{I}^{(5)}-r^{2}\left(\mathcal{S}^{2}+4\right)\right) \mathcal{A}_{a}^{(-)}\left[\phi^{(s)}\right] \\
& =\mathcal{A}_{a}^{(-)}\left[r^{2} C^{2}[\mathfrak{s o}(4,1)] \phi^{(s)}\right]
\end{aligned}
$$

for $a=0, \ldots, 4$. More explicitly, this reads

$$
\begin{aligned}
-\left(\square_{H}+2 r^{2}(2-s)-r^{2}(2 s(s-1)+4)\right) \mathcal{A}_{a}^{(-)}\left[\phi^{(s)}\right] & =\mathcal{A}_{a}^{(-)}\left[\left(-\square_{H}+r^{2} \mathcal{S}^{2}\right) \phi^{(s)}\right] \\
\left(-\square_{H}+2 r^{2} s^{2}\right) \mathcal{A}_{a}^{(-)}\left[\phi^{(s)}\right] & =\mathcal{A}_{a}^{(-)}\left[\left(-\square_{H}+2 r^{2} s(s+1)\right) \phi^{(s)}\right] \\
\square_{H} \mathcal{A}_{a}^{(-)}\left[\phi^{(s)}\right] & =\mathcal{A}_{a}^{(-)}\left[\left(\square_{H}-2 r^{2} s\right) \phi^{(s)}\right] \quad(\mathrm{A} .
\end{aligned}
$$

using $\mathcal{I}^{(5)} \mathcal{A}_{a}^{(-)}\left[\phi^{(s)}\right]=r^{2}(2-s) \mathcal{A}_{a}^{(-)}\left[\phi^{(s)}\right]$, see (A.31). Similarly, the intertwiner property

$$
C^{2}[\mathfrak{s o}(4,1)]^{(\mathrm{ad})}\left\{x^{a}, \phi_{a}^{H(s-1)}\right\}=\left\{x^{a}, C^{2}[\mathfrak{s o}(4,1)]^{(\mathrm{full})} \phi_{a}^{H(s-1)}\right\}
$$

provides us with

$$
\begin{aligned}
\left(\square_{H}-2 r^{2} s(s+1)\right)\left\{x^{a}, \phi_{a}^{H(s-1)}\right\} & =\left\{x^{a},\left(\square_{H}+2 r^{2}(2-s)-r^{2}(2 s(s-1)+4)\right) \phi_{a}^{H(s-1)}\right\} \\
\left(\square_{H}-2 r^{2} s\right)\left\{x^{a}, \phi_{a}^{H(s-1)}\right\} & =\left\{x^{a}, \square_{H} \phi_{a}^{H(s-1)}\right\} .
\end{aligned}
$$

Open Access. This article is distributed under the terms of the Creative Commons Attribution License (CC-BY 4.0), which permits any use, distribution and reproduction in any medium, provided the original author(s) and source are credited.

\section{References}

[1] H. Steinacker, Emergent Geometry and Gravity from Matrix Models: an Introduction, Class. Quant. Grav. 27 (2010) 133001 [arXiv: 1003.4134] [INSPIRE].

[2] S. Minwalla, M. Van Raamsdonk and N. Seiberg, Noncommutative perturbative dynamics, JHEP 02 (2000) 020 [hep-th/9912072] [INSPIRE].

[3] N. Ishibashi, H. Kawai, Y. Kitazawa and A. Tsuchiya, A large $N$ reduced model as superstring, Nucl. Phys. B 498 (1997) 467 [hep-th/9612115] [INSPIRE].

[4] I. Chepelev and A.A. Tseytlin, Interactions of type IIB D-branes from D instanton matrix model, Nucl. Phys. B 511 (1998) 629 [hep-th/9705120] [INSPIRE].

[5] D.N. Kabat and W. Taylor, Linearized supergravity from matrix theory, Phys. Lett. B 426 (1998) 297 [hep-th/9712185] [INSPIRE].

[6] H.C. Steinacker, String states, loops and effective actions in noncommutative field theory and matrix models, Nucl. Phys. B 910 (2016) 346 [arXiv:1606.00646] [INSPIRE].

[7] I. Chepelev, Y. Makeenko and K. Zarembo, Properties of D-branes in matrix model of IIB superstring, Phys. Lett. B 400 (1997) 43 [hep-th/9701151] [INSPIRE].

[8] H. Aoki, S. Iso, H. Kawai, Y. Kitazawa and T. Tada, Space-time structures from IIB matrix model, Prog. Theor. Phys. 99 (1998) 713 [hep-th/9802085] [INSPIRE]. 
[9] Y. Kimura, Noncommutative gauge theories on fuzzy sphere and fuzzy torus from matrix model, Prog. Theor. Phys. 106 (2001) 445 [hep-th/0103192] [INSPIRE].

[10] Y. Kimura, Noncommutative gauge theory on fuzzy four sphere and matrix model, Nucl. Phys. B 637 (2002) 177 [hep-th/0204256] [INSPIRE].

[11] H. Steinacker, Split noncommutativity and compactified brane solutions in matrix models, Prog. Theor. Phys. 126 (2011) 613 [arXiv:1106.6153] [INSPIRE].

[12] S.-W. Kim, J. Nishimura and A. Tsuchiya, Late time behaviors of the expanding universe in the IIB matrix model, JHEP 10 (2012) 147 [arXiv:1208.0711] [INSPIRE].

[13] S.-W. Kim, J. Nishimura and A. Tsuchiya, Expanding (3+1)-dimensional universe from a Lorentzian matrix model for superstring theory in (9+1)-dimensions, Phys. Rev. Lett. 108 (2012) 011601 [arXiv:1108.1540] [INSPIRE].

[14] Y. Ito, J. Nishimura and A. Tsuchiya, Power-law expansion of the Universe from the bosonic Lorentzian type IIB matrix model, JHEP 11 (2015) 070 [arXiv: 1506. 04795] [INSPIRE].

[15] J. Nishimura and A. Tsuchiya, Complex Langevin analysis of the space-time structure in the Lorentzian type IIB matrix model, JHEP 06 (2019) 077 [arXiv: 1904.05919] [INSPIRE].

[16] D.N. Blaschke and H. Steinacker, Curvature and Gravity Actions for Matrix Models, Class. Quant. Grav. 27 (2010) 165010 [arXiv: 1003.4132] [INSPIRE].

[17] H. Steinacker, Gravity and compactified branes in matrix models, JHEP 07 (2012) 156 [arXiv: 1202.6306] [INSPIRE].

[18] H. Steinacker, The curvature of branes, currents and gravity in matrix models, JHEP 01 (2013) 112 [arXiv:1210.8364] [INSPIRE].

[19] H. Kawai, K. Kawana and K. Sakai, A note on graviton exchange in the emergent gravity scenario, PTEP 2017 (2017) 043B06 [arXiv: 1610.09844] [INSPIRE].

[20] V.O. Rivelles, Noncommutative field theories and gravity, Phys. Lett. B 558 (2003) 191 [hep-th/0212262] [INSPIRE].

[21] M. Hanada, H. Kawai and Y. Kimura, Describing curved spaces by matrices, Prog. Theor. Phys. 114 (2006) 1295 [hep-th/0508211] [INSPIRE].

[22] H.S. Yang, Emergent Gravity from Noncommutative Spacetime, Int. J. Mod. Phys. A 24 (2009) 4473 [hep-th/0611174] [INSPIRE].

[23] H.S. Yang and M. Sivakumar, Emergent Gravity from Quantized Spacetime, Phys. Rev. D 82 (2010) 045004 [arXiv:0908. 2809] [INSPIRE].

[24] A. Chaney, L. Lu and A. Stern, Matrix Model Approach to Cosmology, Phys. Rev. D 93 (2016) 064074 [arXiv: 1511.06816] [INSPIRE].

[25] Y. Kaneko, H. Muraki and S. Watamura, Contravariant geometry and emergent gravity from noncommutative gauge theories, Class. Quant. Grav. 35 (2018) 055009 [arXiv:1711.01708] [INSPIRE].

[26] A. Stern and C. Xu, Signature change in matrix model solutions, Phys. Rev. D 98 (2018) 086015 [arXiv: 1808.07963] [INSPIRE].

[27] H.S. Snyder, Quantized space-time, Phys. Rev. 71 (1947) 38 [INSPIRE].

[28] C.N. Yang, On quantized space-time, Phys. Rev. 72 (1947) 874 [INSPIRE].

[29] H. Grosse, C. Klimčík and P. Prešnajder, On finite 4-D quantum field theory in noncommutative geometry, Commun. Math. Phys. 180 (1996) 429 [hep-th/9602115] [INSPIRE]. 
[30] S. Ramgoolam, On spherical harmonics for fuzzy spheres in diverse dimensions, Nucl. Phys. B 610 (2001) 461 [hep-th/0105006] [INSPIRE].

[31] J. Medina and D. O'Connor, Scalar field theory on fuzzy $S^{4}$, JHEP 11 (2003) 051 [hep-th/0212170] [INSPIRE].

[32] J.-P. Gazeau and F. Toppan, A natural fuzzyness of de Sitter space-time, Class. Quant. Grav. 27 (2010) 025004 [arXiv: 0907.0021 ] [INSPIRE].

[33] J. Heckman and H. Verlinde, Covariant non-commutative space-time, Nucl. Phys. B 894 (2015) 58 [arXiv:1401.1810] [INSPIRE].

[34] M. Burić, D. Latas and L. Nenadovic, Fuzzy de Sitter Space, Eur. Phys. J. C 78 (2018) 953 [arXiv: 1709.05158] [INSPIRE].

[35] M. Sperling and H.C. Steinacker, Covariant 4-dimensional fuzzy spheres, matrix models and higher spin, J. Phys. A 50 (2017) 375202 [arXiv:1704.02863] [INSPIRE].

[36] H.C. Steinacker, Emergent gravity on covariant quantum spaces in the IKKT model, JHEP 12 (2016) 156 [arXiv: 1606. 00769] [inSPIRE].

[37] P. de Medeiros and S. Ramgoolam, Non-associative gauge theory and higher spin interactions, JHEP 03 (2005) 072 [hep-th/0412027] [INSPIRE].

[38] M.A. Vasiliev, Higher spin gauge theories in various dimensions, Fortsch. Phys. 52 (2004) 702 [hep-th/0401177] [INSPIRE].

[39] H.C. Steinacker, Quantized open FRW cosmology from Yang-Mills matrix models, Phys. Lett. B 782 (2018) 176 [arXiv:1710.11495] [INSPIRE].

[40] M. Sperling and H.C. Steinacker, The fuzzy 4-hyperboloid $H_{n}^{4}$ and higher-spin in Yang-Mills matrix models, Nucl. Phys. B 941 (2019) 680 [arXiv:1806. 05907] [INSPIRE].

[41] E. Sezgin and P. Sundell, On an exact cosmological solution of higher spin gauge theory, Bulg. J. Phys. 33 (2006) 506 [hep-th/0511296] [INSPIRE].

[42] R. Aros, C. Iazeolla, J. Noreña, E. Sezgin, P. Sundell and Y. Yin, FRW and domain walls in higher spin gravity, JHEP 03 (2018) 153 [arXiv:1712.02401] [INSPIRE].

[43] E. Joung and K. Mkrtchyan, Notes on higher-spin algebras: minimal representations and structure constants, JHEP 05 (2014) 103 [arXiv:1401.7977] [INSPIRE].

[44] H.C. Steinacker, Cosmological space-times with resolved Big Bang in Yang-Mills matrix models, JHEP 02 (2018) 033 [arXiv:1709.10480] [INSPIRE].

[45] D. Klammer and H. Steinacker, Fermions and noncommutative emergent gravity II: Curved branes in extra dimensions, JHEP 02 (2010) 074 [arXiv:0909.5298] [INSPIRE].

[46] A.D. Sakharov, Vacuum quantum fluctuations in curved space and the theory of gravitation, Sov. Phys. Dokl. 12 (1968) 1040 [InSPIRE].

[47] N. Berline, E. Getzler and M. Vergne, Heat kernels and Dirac operators, Grundlehren Text Editions, Springer-Verlag, Berlin, Germany, (2004).

[48] K. Hasebe, Non-Compact Hopf Maps and Fuzzy Ultra-Hyperboloids, Nucl. Phys. B 865 (2012) 148 [arXiv:1207.1968] [INSPIRE].

[49] G. Mack, All unitary ray representations of the conformal group $\mathrm{SU}(2,2)$ with positive energy, Commun. Math. Phys. 55 (1977) 1 [INSPIRE].

[50] S. Fernando and M. Günaydin, Minimal unitary representation of $\mathrm{SU}(2,2)$ and its deformations as massless conformal fields and their supersymmetric extensions, J. Math. Phys. 51 (2010) 082301 [arXiv:0908.3624] [INSPIRE]. 
[51] G. Mack and I. Todorov, Irreducibility of the ladder representations of $\mathrm{U}(2,2)$ when restricted to the Poincaré subgroup, J. Math. Phys. 10 (1969) 2078 [InSPIRE].

[52] W. Heidenreich, Tensor Products of Positive Energy Representations of $\mathrm{SO}(3,2)$ and $\mathrm{SO}(4,2)$, J. Math. Phys. 22 (1981) 1566 [inSPIRE].

[53] A.M. Perelomov, Generalized coherent states and their applications, Springer, Berlin Heidelberg, Germany (1986).

[54] H. Grosse and P. Prešnajder, The construction on noncommutative manifolds using coherent states, Lett. Math. Phys. 28 (1993) 239 [INSPIRE].

[55] E. Hawkins, Quantization of equivariant vector bundles, Commun. Math. Phys. 202 (1999) 517 [q-alg/9708030] [INSPIRE].

[56] G. Ishiki, Matrix Geometry and Coherent States, Phys. Rev. D 92 (2015) 046009 [arXiv: 1503.01230] [INSPIRE].

[57] G. Ishiki, T. Matsumoto and H. Muraki, Information metric, Berry connection and Berezin-Toeplitz quantization for matrix geometry, Phys. Rev. D 98 (2018) 026002 [arXiv: 1804.00900] [INSPIRE].

[58] J. Pawełczyk and H. Steinacker, A quantum algebraic description of D branes on group manifolds, Nucl. Phys. B 638 (2002) 433 [hep-th/0203110] [INSPIRE].

[59] N. Seiberg and E. Witten, String theory and noncommutative geometry, JHEP 09 (1999) 032 [hep-th/9908142] [INSPIRE].

[60] E.W. Kolb, A Coasting Cosmology, Astrophys. J. 344 (1989) 543 [InSPIRE].

[61] M.V. John and K.B. Joseph, Generalized Chen-Wu type cosmological model, Phys. Rev. D 61 (2000) 087304 [gr-qc/9912069] [inSPIRE].

[62] F. Melia and A. Shevchuk, The $R_{h}=$ ct Universe, Mon. Not. Roy. Astron. Soc. 419 (2012) 2579 [arXiv: 1109.5189] [INSPIRE].

[63] M.R. Douglas and N.A. Nekrasov, Noncommutative field theory, Rev. Mod. Phys. 73 (2001) 977 [hep-th/0106048] [INSPIRE].

[64] R.J. Szabo, Quantum field theory on noncommutative spaces, Phys. Rept. 378 (2003) 207 [hep-th/0109162] [INSPIRE].

[65] M. Sperling and H.C. Steinacker, Intersecting branes, Higgs sector and chirality from $\mathcal{N}=4$ SYM with soft SUSY breaking, JHEP 04 (2018) 116 [arXiv: 1803.07323] [INSPIRE].

[66] H. Aoki, J. Nishimura and A. Tsuchiya, Realizing three generations of the Standard Model fermions in the type IIB matrix model, JHEP 05 (2014) 131 [arXiv:1401.7848] [INSPIRE].

[67] D.N. Blaschke and H. Steinacker, On the 1-loop effective action for the IKKT model and non-commutative branes, JHEP 10 (2011) 120 [arXiv:1109.3097] [INSPIRE].

[68] M. Visser, Sakharov's induced gravity: A modern perspective, Mod. Phys. Lett. A 17 (2002) 977 [gr-qc/0204062] [inSPIRE].

[69] P. Gilkey, Invariance Theory: The Heat Equation and the Atiyah-Singer Index Theorem, CRC-Press, (1995).

[70] H.C. Steinacker, One-loop stabilization of the fuzzy four-sphere via softly broken SUSY, JHEP 12 (2015) 115 [arXiv:1510.05779] [INSPIRE]. 